\title{
The biological control potential of Cryphonectria parasitica strains containing an infectious cDNA copy of hypovirus CHV1-Euro7
}

William R. Rittenour

West Virginia University

Follow this and additional works at: https://researchrepository.wvu.edu/etd

\section{Recommended Citation}

Rittenour, William R., "The biological control potential of Cryphonectria parasitica strains containing an infectious cDNA copy of hypovirus CHV1-Euro7" (2005). Graduate Theses, Dissertations, and Problem Reports. 2291.

https://researchrepository.wvu.edu/etd/2291

This Thesis is protected by copyright and/or related rights. It has been brought to you by the The Research Repository @ WVU with permission from the rights-holder(s). You are free to use this Thesis in any way that is permitted by the copyright and related rights legislation that applies to your use. For other uses you must obtain permission from the rights-holder(s) directly, unless additional rights are indicated by a Creative Commons license in the record and/ or on the work itself. This Thesis has been accepted for inclusion in WVU Graduate Theses, Dissertations, and Problem Reports collection by an authorized administrator of The Research Repository @ WVU. For more information, please contact researchrepository@mail.wvu.edu. 
The biological control potential of Cryphonectria parasitica strains containing an infectious cDNA copy of hypovirus CHV1-Euro7

\author{
William R. Rittenour \\ Thesis submitted to the \\ College of Agriculture, Forestry and Consumer Sciences \\ At West Virginia University \\ In partial fulfillment of the requirements \\ For the degree of \\ Master of Science \\ in \\ Plant Pathology \\ William MacDonald, Ph.D., Chair \\ John Brooks, Ph.D. \\ Donald Nuss, Ph.D. \\ Daniel Panaccione, Ph.D. \\ Department of Plant Pathology and Environmental Microbiology \\ Morgantown, WV \\ 2005
}

Keywords: chestnut blight, hypovirulence, transgenic 


\section{Abstract \\ The biological control potential of Cryphonectria parasitica strains containing an infectious cDNA copy of hypovirus CHV1-Euro7}

\section{William R. Rittenour}

Transmissible hypovirulence has not become established in most areas of North America as it has in Europe where it has been associated with increased biological control of chestnut blight. Transgenic strains of Cryphonectria parasitica provide several mechanisms that may improve biological control. This study was designed to evaluate whether transgenic $C$.

parasitica strains containing a cDNA transgene encoding the viral genome of CHV1-Euro7 show greater potential to biologically control blight than their cytoplasmically infected counterparts. Three treatments were employed that compared: transgenic hypovirulent strains (TG), cytoplasmic hypovirulent strains (CH), and virulent strains (V). Two types of cankers were initiated in each treatment to generate inoculum. To produce ascospore inoculum, naturally occurring and artificially established cankers were spermatized by painting cankers in June, July, and September with a conidial mixture that contained both mating types (MAT-1 and MAT-2) of the appropriate treatment strain (TG, $\mathrm{CH}$, or V). To produce conidial inoculum, cankers were scratch-initiated (SI) on separate trees in June 2004 by scratching the surface of the bark and painting the wounded area with a mycelial-agar slurry of the appropriate treatment strain (TG, $\mathrm{CH}$, or V). Non-treated trees also were left to monitor natural canker formation. In October, 2004, tree condition and natural canker establishment were assessed for all trees. Most trees were asymptomatic after the first treatment season and the incidence of natural infection remained relatively low. There were 27 natural cankers in TG plots, 13 cankers in CH plots, and 20 cankers in V plots. Cankers also were sampled to determine the hypovirus infection status of the thallus. Although the purpose of the spermatization treatment was to produce ascospores, many treated cankers also acquired hypovirus from the treatment inoculum. Cankers occurring below SI cankers also acquired hypovirus. Ascospore production was assessed by collecting bark discs in October 2004 and serially diluting ascospore contents from perithecia in the winter of 2004-2005. Pigmentation and morphology then were recorded for ascospores from all three plot treatments (TG, V, and CH). Hypovirulent ascospore (HVA) isolates were only collected from TG plots and at less than expected Mendelian ratios. Pigmentation segregated as expected in $\mathrm{V}$ and $\mathrm{CH}$ plots. The transgenic MAT-1 treatment strain effectively spermatized and hence produced HVAs on both initiated cankers and treated natural infections. The transgenic MAT-2 treatment strain did not produce morphologically distinct HVA isolates. To assess the increased conversion capability of HVAs, 18 HVA isolates were paired with 17 vegetative compatibility $(\mathrm{v}-\mathrm{c})$ types isolated from the study site; pairings then were examined for hypovirus transmission. Collectively, HVA isolates consistently (i.e. 4-5 conversions out of 5 replications) converted 12 of the v-c types; three additional v-c types were able to be converted at least once. When conidial inoculum produced by SI cankers was analyzed, the average percentage of hypovirulent conidia harvested was $~ 98 \%$ in TG plots, while no viable pycnidia (asexual fruiting bodies) were harvested from $\mathrm{CH}$ plots. Three of the $4 \mathrm{SI}$ cankers analyzed from the V plots yielded all $\mathrm{V}$ conidia (i.e. 0\% HV), but the remaining canker yielded 80\% HV conidia. Overall, the production of HVAs with different conversion capabilities increased the biological control potential of transgenic strains. Monitoring canker development in subsequent years will provide further information on the fate of the transgenic inoculum being produced and whether this approach results in improved biological control. 


\section{Acknowledgements}

There are many people that made this study possible. First I would like to thank APHIS for granting permission to release a transgenic organism into the "wild." I also would like to thank the USDA Forest Service for allowing us to establish the study site in the Monongahela National Forest in Pendleton, WV. Also, I would like to thank everyone who helped me set apply treatments and collect data: Jenise Bauman, Jessica Bright, and Christian Root. Thank you to Christine Coyle for assistance with molecular techniques. Many thanks to Dr. Michael Milgroom of Cornell University and Dr. Bradley Hillman of Rutgers University for their input into aspects of this study. I would like to thank my committee members John Brooks, Donald Nuss, and Daniel Panaccione for their input and support. Great thanks to Lynn Geletka and Dr. Nuss for their work in engineering the strains that made this study possible. My deepest gratitude to Mark Double for his patience and understanding in teaching delicate lab techniques to a trained forester. I owe much of my academic progress over the past two years to my advisor Dr. William MacDonald. His patience, friendship, and good humor kept a positive atmosphere even in stressful times. I would finally like to extend my deepest appreciation to my sisters Alicia and Tricia, my wife Christy, and my parents Erroll, Theresa, Craig, and Cathy. I would not be where I am today without your mental and financial support. 


\section{Table of Contents}

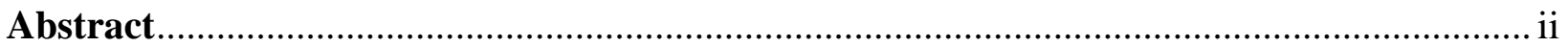

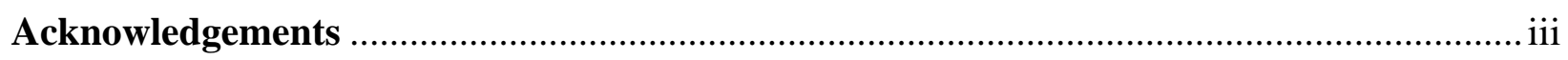

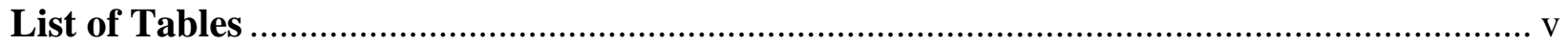

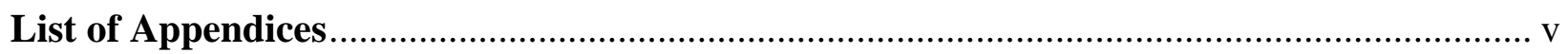

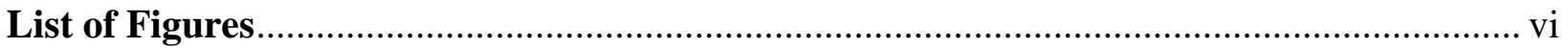

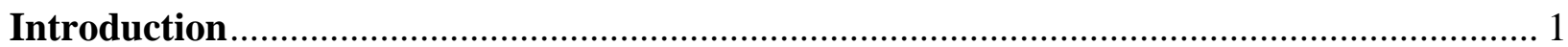

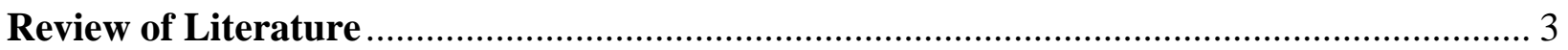

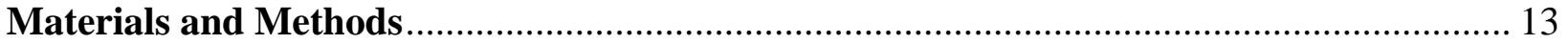

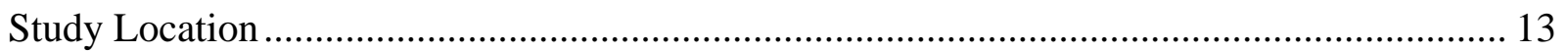

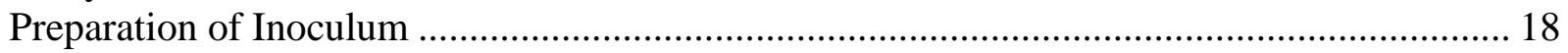

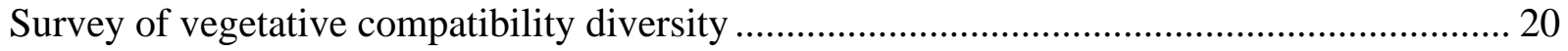

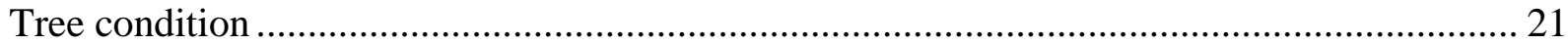

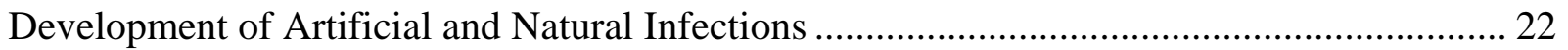

Sampling Artificial and Natural Cankers..................................................................... 22

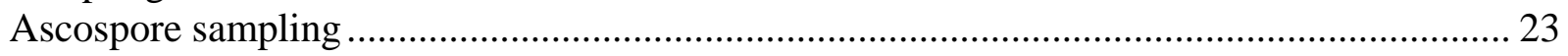

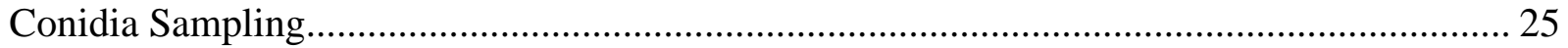

Double-stranded RNA Extraction and Pathogenicity Testing ........................................... 26

Evaluation of the Potential for hypovirus transfer to other v-c types ................................... 26

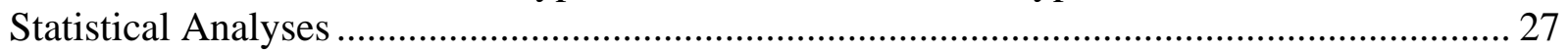

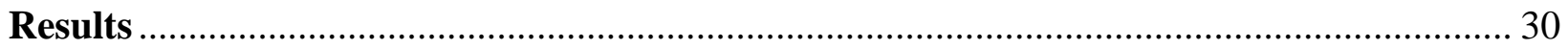

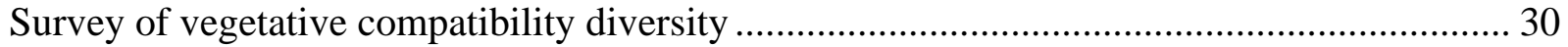

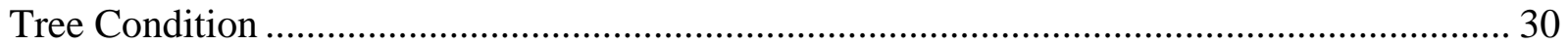

Development of Artificial and Natural Infections .......................................................... 31

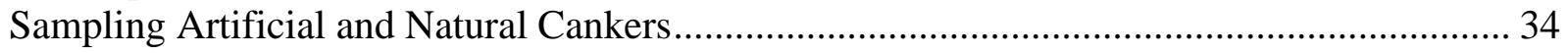

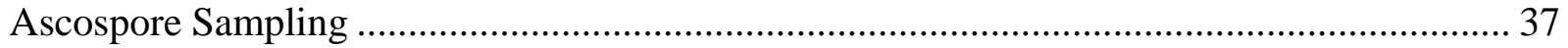

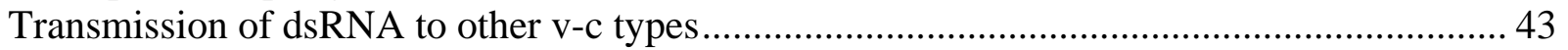

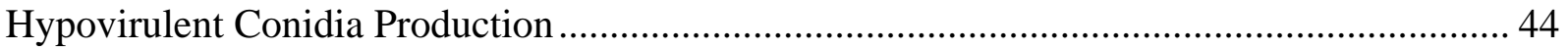

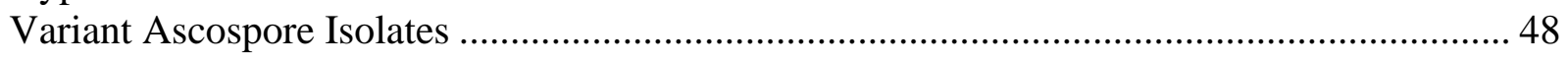

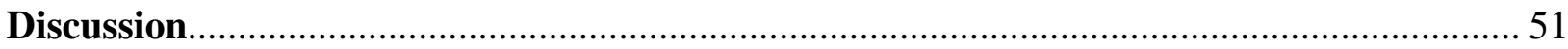

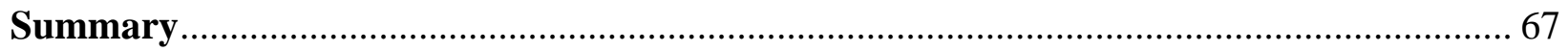

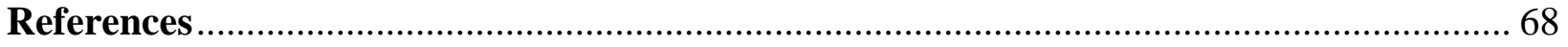

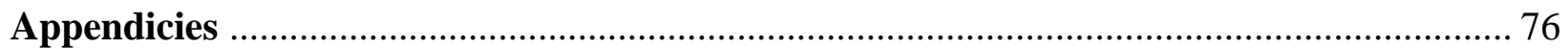




\section{List of Tables}

Table 1. Cryphonectria parasitica isolates employed in this study....................................... 16

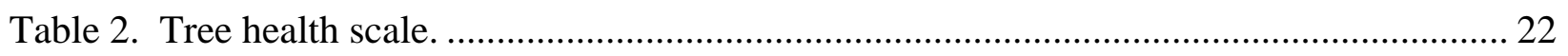

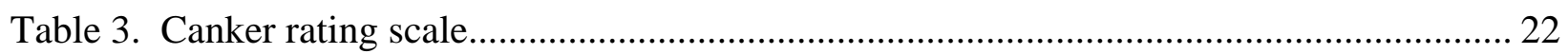

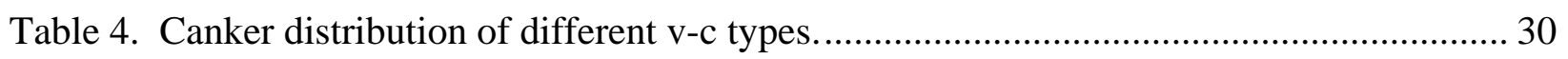

Table 5. Average tree health rating ${ }^{1}$ in May and August, 2004.............................................. 31

Table 6. Number of natural cankers discovered in May and October of the 2004 field season. 31

Table 7. HV recovery from cankers in V plots................................................................. 36

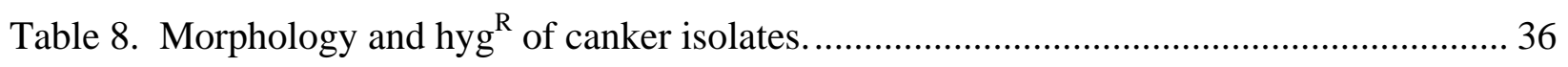

Table 9. Outcrossing percentages for three main treatments by canker and perithecia levels.... 39

Table 10. Number of ascospore colonies isolated from PI and natural cankers....................... 42

Table 11. Ratios of hyg ${ }^{\mathrm{R}}$ :hyg ${ }^{\mathrm{S}}$ ascospores from EP155/pxHE7 crosses ................................ 43

Table 12. Number of hypovirus transmissions from selected hypovirulent ascospores to selected

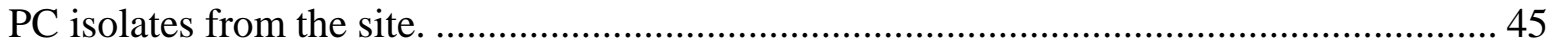

\section{$\underline{\text { List of Appendices }}$}

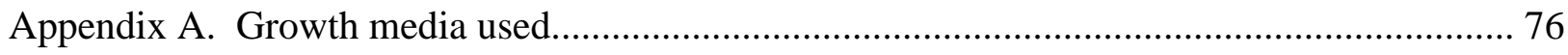

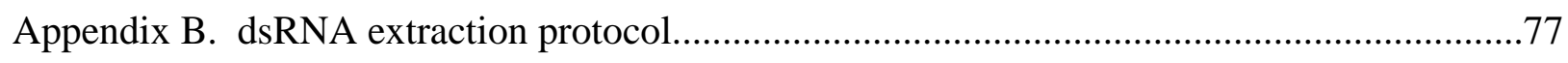

Appendix C. ANOVA tables from split-plot statistical analyses........................................78 


\section{$\underline{\text { List of Figures }}$}

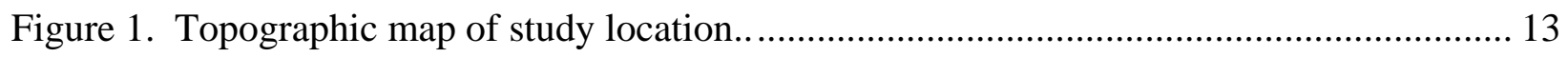

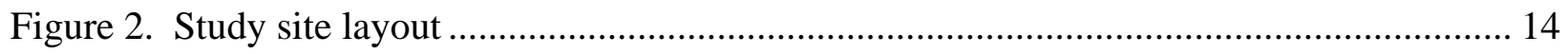

Figure 3. Cryphonectria parasitica strains that were released in the field study..................... 15

Figure 4. Techniques used to scratch-initiate cankers ..................................................... 17

Figure 5. Initiation and spermatization of punch-initiated cankers.. ..................................... 18

Figure 6. Collection of bark plugs in microtiter dish with bone marrow instrument. ................ 20

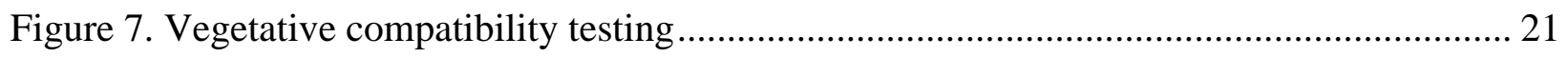

Figure 8. Procedure for isolating C. parasitica from bark plugs .......................................... 23

Figure 9. Removal and storage of bark discs collected for sampling perithecia ....................... 25

Figure 10. Procedure used for isolating ascospore colonies from perithecia ............................ 25

Figure 11. Non-converted (above) and converted (below) isolates....................................... 27

Figure 12. Scratch-initiated cankers after removal of cover and in October 2004.................... 32

Figure 13. Expansion of scratch-initiated cankers ............................................................. 32

Figure 14. Average canker ratings of initiated and natural cankers in October, 2004 .............. 33

Figure 15. Average canker rating for treated and non-treated cankers in October, 2004........... 33

Figure 16. Average rating of cankers from different HV recovery classes ............................. 34

Figure 17. Number of colonies by morphology collected from bark plugs on four types of

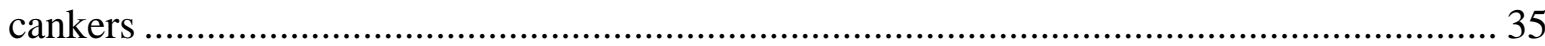

Figure 18. Average number of HV isolates recovered from PI cankers and number of times PI canker inciting strains were converted under laboratory conditions ................................ 38

Figure 19. Observed and expected values for ascospore progeny from EP146 crosses in each of

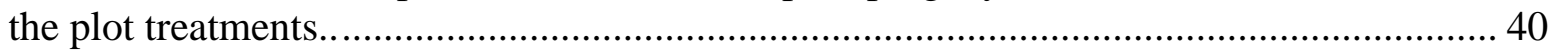

Figure 20. Dissemination of cDNA to different v-c groups after sexual recombination............ 44 
Figure 21. Percentages of conidial isolates by morphology collected from scratch-initiated

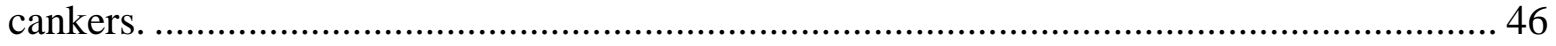

Figure 22. Morphologies of EP155/pxHE7 conidia isolations done in February, 2005 ............... 47

Figure 23. Apple lesion diameters after inoculation with various EP155/pxHE7 conidia isolates......................................................................................... 48

Figure 24. Ascospore colonies showing variation in morphology.............................................. 49

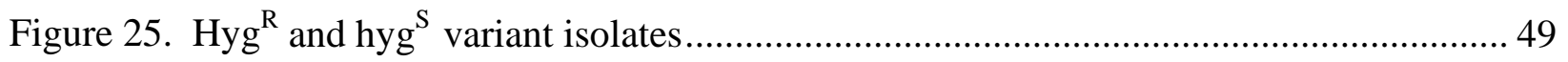

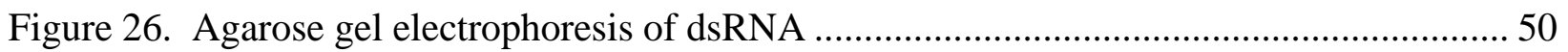




\section{Introduction}

Chestnut blight, caused by the fungus Cryphonectria parasitica, eliminated most mature American chestnut (Castanea dentata) trees in eastern North America. Attempts to control the blight have focused on either breeding for resistance or biological control utilizing fungal dsRNA hypoviruses. Strains infected with dsRNA hypoviruses are less virulent ("hypovirulent”) to chestnut and provide a mechanism for biological control because they can transmit their hypoviruses to virulent strains via anastomosis (hyphal fusion), thereby "converting” the virulent strain to hypovirulent. The success of hypoviruses at blight control is evident in Europe where chestnut (C. sativa) stands are recovering; comparable biological control has not occurred in most areas of North America. The population structure of C. parasitica is more complex in N. America than in Europe and harbors a diverse group of vegetative compatibility (v-c) types. This complex system of v-c types may restrict the transmission and dissemination of hypoviruses in N. American forests.

Transgenic strains of $C$. parasitica that contain a complementary DNA (cDNA) copy of the dsRNA viral genome provide advantages that may enhance biological control over "classic" cytoplasmic hypovirulent strains. Cytoplasmic hypovirulent strains transmit their hypovirus to conidia (asexual spores) at variable rates and do not transmit their hypovirus to ascospores (sexual spores). Alternatively, transgenic strains transmit their hypovirus to $\sim 100 \%$ conidia and $\sim 50 \%$ ascospores. Transmission of the cDNA gene to ascospore progeny also results in dissemination of dsRNA to a variety of vegetative incompatibility groups, hence increasing the potential of hypovirus transmission to virulent strains.

The hypovirus used for transgenic studies thus far (CHV1-Ep713) is very debilitating to host fungus and potentially restricts establishment and dissemination of hypovirulent strains. 
The objective of this study was to evaluate the biological control potential of transgenic strains infected with the mildly debilitating hypovirus CHV1-Euro7. 


\section{$\underline{\text { Review of Literature }}$}

The American chestnut (Castanea dentata (Marsh.) Borkh.) once was a major component of the eastern hardwood forest before arrival of the chestnut blight fungus, Cryphonectria parasitica (Kuhlman, 1978). The tree accounted for 25\% of the hardwood trees within the 200 million acre natural range in eastern North America (Braun, 1950). This abundant species had many qualities that made it valuable to both humans and wildlife. Its straight bole, rapid growth and resistance to decay made it a valuable timber resource that was used for many products including telephone poles, ship masts and railroad ties (Kuhlman, 1978). The nuts of American chestnut are sweeter than other chestnut species and were not only popular for human consumption, but the steady yearly crop also provided a staple in the diet of wild turkeys, squirrels, jays, bears, and deer (Newhouse, 1990; Ronderos, 2000). The chestnut blight fungus eliminated most of the estimated 3.5 billion overstory chestnut trees in the Appalachians by 1950, and now the species can only be found growing as stump sprouts (Hebard, 1982). The sprouts grow until they contract the disease and the cycle of regrowth and infection repeats itself.

Chestnut blight is caused by an ascomycete fungus that was first discovered in the U.S. on American chestnut shade trees in New York City in 1904 (Kuhlman, 1978). The fungus originally was named Diaporthe parasitica by Murrill (1906), a pioneer in plant pathology who investigated and attempted to control the blight. The name soon was changed to Endothia parasitica by Anderson and Anderson (1912). The genus name again was changed in 1978 to its current classification Cryphonectria parasitica based on the physical structure of its perithecia (Barr, 1978).

Cryphonectria parasitica was thought to be introduced into the U.S. on ornamental Asian chestnut species (C. crenata Sieb. and Zucc. and C. mollissima Bl.) (Anagnostakis, 1987; Roane 
et al, 1986). Scientists observed early on that Asian species were more resistant to blight; it was suspected that the fungus and Asian species co-evolved in their native range. Their suspicion was confirmed as early as 1913 when plant explorer F. Meyer found the disease in both China and Japan and sent samples back to the U.S. for analysis. The samples were very similar to the strain infecting American chestnut (Shear and Stevens, 1916). Recent genetic analyses suggest that most C. parasitica strains were introduced from Japan (Milgroom, 1995; Milgroom et al, 1996).

Cryphonectria parasitica affects healthy chestnut trees when fungal propagules (ascospores, conidia, or hyphae) colonize a bark wound. In order for sexual reproduction to occur, spermatia and trichogynes (female receptive hyphae) must be of opposite mating types designated MAT-1 and MAT-2 (Arie et al., 1997). Ascospores are expelled from perithecia (sexual fruiting bodies) and are thought to be wind-disseminated while conidia (asexual spores) are produced in sticky tendrils and are disseminated locally by insects and birds (Sharf and DePalma, 1981; Russin and Shain, 1983; Diamandis and Perlerou, 2005). A germinating spore eventually becomes a mycelial fan that grows into the inner cortex and cambium; if the mycelial fan grows large enough and is unchecked by tree defenses, it eventually forms a canker that girdles and kills the stem or tree depending on canker location (Murrill, 1906; Roane et al., 1986). This girdling results in the yellowing and dying of leaves, the symptom for which the disease gets its name (Roane et al., 1986). Numerous orange stromata may be evident in areas where the fungus is growing (Murrill, 1906). The expanding fungus, however, will not advance into the roots, allowing sprouts to grow from stumps of dead trees (Griffin, 1986). Cryphonectria parasitica can infect Allegheny chinquapin (Castanea pumila) and many oak species (Quercus spp.), although it is usually not as virulent on these species (Griffin, 1986). 
Efforts to control the disease began soon after its discovery in 1904, though the early rapid spread of the blight lead to a pessimistic outlook. Murrill (1906) suggested that the mycelia spread so fast that the only action to be recommended was the "rigorous use of a pruning knife.” The hope was to prune branches below the infection to stop the disease spread and then cover the resulting wound with tar to prevent further colonization. This method would obviously not work if the bole of the tree was already infected and the procedure had no practical use in forest settings. Murrill (1906) also ruled out spraying solutions because they would not reach the fungus inside the bark or reduce the countless number of spores produced throughout the year. Early efforts to control the disease in forest settings focused on felling and removing infected trees in disease centers and sometimes burning the bark and brush on stumps (Kuhlman, 1978). The State of Pennsylvania formed the Chestnut Blight Commission in 1911 and empowered it "to use all practical means to destroy the blight," including the right to enter any property, regardless of ownership, and remove diseased or threatened trees (Hepting, 1974). Though a spirited effort was put forth, the chestnut blight fungus spread unabated through the Appalachians by spore dissemination and new nursery introductions until virtually all areas of chestnut's natural range were infected (Kuhlman, 1978).

While chemical injections and compost/soil applications to cankers have proven somewhat effective at controlling the disease in orchard and ornamental settings, the two main methods of control that offer promise in restoring the American chestnut to forest settings are breeding for resistance and biological control utilizing hypoviruses (Roane et al., 1986; Griffin, 1986; Griffin, 2000). Currently, the American Chestnut Foundation is conducting genetic crosses between American chestnuts and Asian species to produce offspring that are more resistant (Hebard, 2002). Offspring are then "backcrossed" to American chestnut repeatedly to 
maintain as many traits of the American species as possible, while retaining the resistance conferred by the Asian species (Burnham et al., 1986; Ellingboe, 1992).

Hypovirulent strains of $C$. parasitica show promise as control agents because they are less damaging to chestnut trees (Van Alfen et al., 1975; Anagnostakis and Jaynes, 1973; Elliston, 1978). These strains produce fewer pycnidia (asexual fruiting bodies) and cause smaller, less invasive cankers (sometimes called "superficial” cankers) in which the fungus usually does not penetrate to the cambial layer of the tree (Elliston, 1978). Trees are able to compartmentalize these superficial cankers before the fungus spreads to the cambium and girdles the tree (Jaynes and Elliston, 1982; Hebard et al., 1984). Hypovirulent strains of the fungus were first discovered in coppice stands of European chestnut (C. sativa Mill.) in Italy in 1950, 12 years after the blight epidemic began in Europe (Mittempergher, 1978). Early researchers noticed that these strains were not only less virulent but also had the ability to transmit the hypovirulence factor to virulent strains when hyphae of the two fused by anastomosis (Anagnostakis and Day, 1979). This led researchers to believe that hypovirulence was probably caused by genetic determinants present in the cytoplasm. The suspicion was later confirmed by the discovery of viral double-stranded RNAs (dsRNAs) in most hypovirulent strains (Moffitt and Lister, 1975; Morris and Dodds, 1979; Day et al., 1977; Van Alfen, 1975).

Double-stranded RNA fragments have been associated with virulence attenuation in many plant pathogenic fungi (reviewed by Nuss and Koltin, 1990). In C. parasitica, the presence of dsRNA fragments disturbs multiple regulatory pathways and often leads to symptoms such as reduced pigmentation, reduced sporulation, and reduced growth (Chen et al., 1996; Dawe and Nuss, 2001). The presence of dsRNA fragments also causes reduced 
conidiation and virulence when introduced into species closely related to C. parasitica (Chen et al., 1994; Chen et al., 1996).

These dsRNA fragments share some common features with viruses but are not encapsulated by structural proteins. They are classified in the family Hypoviridae (Hillman et al., 2000) and are commonly referred to as “hypoviruses”. Four distinct Cryphonectria hypoviruses (CHV1-CHV4) have been identified, which vary in genome organization and effect on host virulence (Hillman and Suzuki, 2004).

The effect a hypovirus has on its host varies depending on the species of hypovirus and the genetic background of the host. Before the discernment of hypovirus species, Russin and Shain (1985) demonstrated that hypovirulence-causing agents from different parts of the world (presumably representing what is now known as different hypoviruses) varied in their ability to reduce virulence, transmit hypoviruses, and produce hypovirulent conidia. Elliston (1985) also found that dsRNA-containing strains from France, Italy, and North America all had varying effects on canker development and sporulation on dormant chestnut stems and live trees. After the classification of hypoviruses, Chen and Nuss (1999) found significantly reduced canker growth and sporulation on C. parasitica strains infected with CHV1-EP713 than those infected with CHV1-Euro7.

The utilization of hypovirulence as a biological control relies on the ability of the hypovirulent strains to disseminate and transmit their hypovirus to virulent strains. Hypovirulent strains can disseminate locally through the dispersal of conidia that inherit dsRNA fragments (Elliston, 1978). Hypoviruses also can be transmitted from hypovirulent to virulent strains through hyphal anastomosis, thereby “converting” virulent strains to hypovirulent (Van Alfen et al., 1975; Anagnostakis, 1983). 
In less than 15 years after the discovery of hypovirulence, the severity of chestnut blight naturally decreased in regenerating coppice stands of C. sativa in Italy (Mittempergher, 1978). This prompted American researchers to test hypovirulent strains from Europe as biological control agents (Anagnostakis, 1982; Jaynes and DePalma, 1982; Van Alfen et al., 1975; Jaynes and Elliston, 1978; Jaynes and Elliston, 1980). Though some level of biological control was demonstrated on single cankers, hypovirulent strains did not disseminate as well in North America as they did in Europe (MacDonald and Fulbright, 1991; Willey, 1982; Heiniger and Rigling, 1994).

One factor that may limit dissemination of hypovirulent strains is the transmission of hypovirus to offspring. In a series of experiments performed by Melzer et al. (1997), rates of hypovirus transmission to conidia ranged from $0-100 \%$ across eight different hypovirulent strains, with a mean rate of approximately $40 \%$. Converted cankers also have been shown to continue producing virulent conidia, even when the underlying thallus yielded hypovirulent isolates (Shain and Miller, 1992). Furthermore, hypovirulent strains are female infertile, meaning they lack functioning trichogynes and do not produce perithecia (Elliston, 1978; Elliston, 1985). Though hypovirulent conidia can spermatize the trichogyne of a virulent strain, the resulting perithecia do not contain hypovirulent ascospores (Anagnostakis, 1984; Fulbright et al., 1988). Many of the hypovirulent strains released in North America were the same strains that have demonstrated success in Europe, suggesting that hypovirus transmission to spores is not the most limiting factor in dissemination.

Another barrier to dissemination may be the failure of hypovirus transmission caused by vegetative incompatibility. Anastomosis between two strains of $C$. parasitica is dictated by the genetic composition at six vegetative incompatibility (vic) loci (Cortesi and Milgroom, 1998; 
Cortesi et al., 2001). Strains must have identical alleles at all six vic loci in order to undergo stable anastomosis. However, the composition of vic alleles is less stringent on the process of conversion (Anagnostakis, 1983; Cortesi et al., 2001). Conversion between two strains varies depending on which loci differ and whether the donor or receiver has a particular allele. For example, Cortesi et al. (2001) demonstrated that conversion regularly occurs when the donor has vic allele 1 and the receiver has vic allele 2 for the vic1 locus, but when the allele situation is reversed for the same locus, conversion rarely occurs.

A high number of different vegetative compatibility (v-c) types limits the occurrence of hyphal fusion and therefore limits the transmission of hypoviruses. Many recovering stands in Europe contain fewer v-c types when compared to areas in North America (Anagnostakis et al., 1986; Bissegger et al., 1997; Milgroom and Cortesi, 1999). Some stands occurring in Michigan outside the native range of American chestnut also are recovering (Fulbright et al., 1983). Some of these stands harbor clonal populations of $C$. parasitica that may facilitate the transmission of hypoviruses (Liu et al., 1996). In the Monongahela National Forest, v-c types were found to be aggregated on the same tree, but random from tree-to-tree (Milgroom et al., 1991). This random distribution of v-c types may inhibit dissemination of hypovirulent strains on a stand level. An ecological model developed by Liu et al. (2000) also showed a negative correlation between v-c type diversity and hypovirus dissemination.

Choi and Nuss (1992) developed a procedure for introducing hypoviruses into $C$. parasitica that may improve the dissemination and biological control potential of hypovirulent strains. This procedure involves the integration of a complementary DNA (cDNA) copy of CHV1-EP713 RNA into the genome of a virulent C. parasitica strain by means of a plasmid vector. The cDNA is then transcribed to produce hypovirus coding strand RNA that is 
transported to the cytoplasm where viral RNA replication is initiated. Strains of C. parasitica transformed by this procedure, termed transgenic hypovirulent strains, contain both nuclear cDNA and cytoplasmic RNA copies of the hypovirus RNA. The plasmid vector also contains a hygromycin resistance $\left(\right.$ hyg $^{\mathrm{R}}$ ) gene which allows for selection of transgenic isolates by screening on hygromycin-containing media.

Nuclear copies of a hypovirus cDNA (not present in “classic” hypovirulent strains) provide significant advantages to biological control that are not present with other hypovirulent strains. These advantages include nearly 100\% hypovirulent conidia production (as opposed to an average of 40\% reported by Melzer et al., (1997)) and a theoretical 50\% hypovirus transmission rate to ascospore progeny (Nuss, 1992a). Rearrangement of vic alleles during meiosis also permits viral cDNA copies to be transmitted to other v-c types represented among the sexual offspring, increasing the possibility of viral dissemination to a great number of v-c types (Nuss, 1992a).

Since the development of transgenic hypovirulent strains, their mitotic and meiotic stability have been demonstrated in the laboratory. Chen et al. (1993) found a 99.4\% hypovirus transmission rate from parent to conidia after 3 asexual generations and about a 50\% transmission rate to ascospore progeny. The authors tested the parental hypovirulent strains and ascospore progeny against $15 \mathrm{v}$-c groups to confirm potential of sexual offspring to carry the virus to other v-c types. While the parental strains were only able to convert four tester strains, the 18 ascospore progeny were able to collectively convert 14 of the 15 tester strains. All ascospore progeny exhibiting hypovirulent traits also displayed hyg ${ }^{\mathrm{R}}$, illustrating uninterrupted inheritance of both hyg ${ }^{\mathrm{R}}$ gene and the cDNA copy of the hypovirus. Southern analysis confirmed that the cDNA insert was present in each of these hyg ${ }^{\mathrm{R}}$ ascospore isolates. 
There also have been studies that examined the persistence of transgenic strains in situ. Anagnostakis et al. (1998) released a transgenic strain of C. parasitica into a Connecticut forest and examined hypovirus transmission to ascospore progeny, dissemination of transgenic strains, and effects of canker treatment on canker growth. In their study, perithecia examined from artificially inoculated virulent cankers treated with transgenic conidia (acting as spermatia) contained transgenic ascospores at rates from $0-63 \%$ per canker, which enabled transmission of viral dsRNA to other v-c types. Transgenic conidia also were shown to germinate and convert virulent strains of similar v-c types. Two new cankers were found to contain viral dsRNA: one transgenic and one cytoplasmic. In a second transgenic field trial, Root et al. (2005) had better hypovirulent ascospore production when the conidial treatment was painted on the canker face, but tree condition in the release areas was not improved over controls.

Though some of the advantages of transgenic strains have been seen in the field, an ecological model developed by Liu et al. (2000) predicted only marginally greater virus dissemination through use of transgenic strains. Resident v-c type diversity still acted as a barrier to virus dissemination in transgenic release models. The transgenic release model also predicted that biological control would not become autonomous and frequent releases of transgenic strains were necessary to maintain slight biological control.

The utility of transgenic hypovirulent strains of $C$. parasitica must be tested further to judge their potential as biological control agents. To date, field experiments performed with transgenic strains have used a cDNA copy of the CHV1-EP713 hypovirus. This hypovirus greatly reduces bark colonization and sporulation of the fungus, more so than other hypoviruses. An ideal hypovirulent strain for biological control should show a balance between virulence attenuation and disseminative potential. Other hypoviruses, such as CHV1-Euro7, are less 
debilitating to their hosts thereby allowing formation of larger cankers that generate more hypovirulent inoculum. Therefore, strains infected with CHV1-Euro7 may provide more efficient biological control through potentially higher dissemination and better establishment, compared to the highly debilitating CHV-EP713.

\section{Objective}

The objective of this study was to evaluate the biological control potential of transgenic C. parasitica strains containing a cDNA copy of the mild hypovirus CHV1-Euro7. This study included aspects that considered: a) the artificial establishment of transgenic hypovirulent cankers and their subsequent inoculum production; b) the spermatizing potential of transgenic conidia; and, c) the ability of transgenic CHV1-Euro7 strains to disseminate. 


\section{Materials and Methods}

\section{Study Location}

This study was conducted in Pendleton County, West Virginia, at a site in the Monongahela National Forest accessed by Forest Service road 48 (Fig. 1). The site had been cut-over but a limited oak overstory remained with abundant chestnut regeneration and low-tomoderate levels of chestnut blight. The site was in a non-populated area thus reducing the chance of human intervention. Site coordinates were 17 S 0624317 UTM 4272289 (N $38^{\circ} 35^{\prime}$ 39.8” W 079 34’ 16.1”) USGS quadrangle: Snowy Mountain.

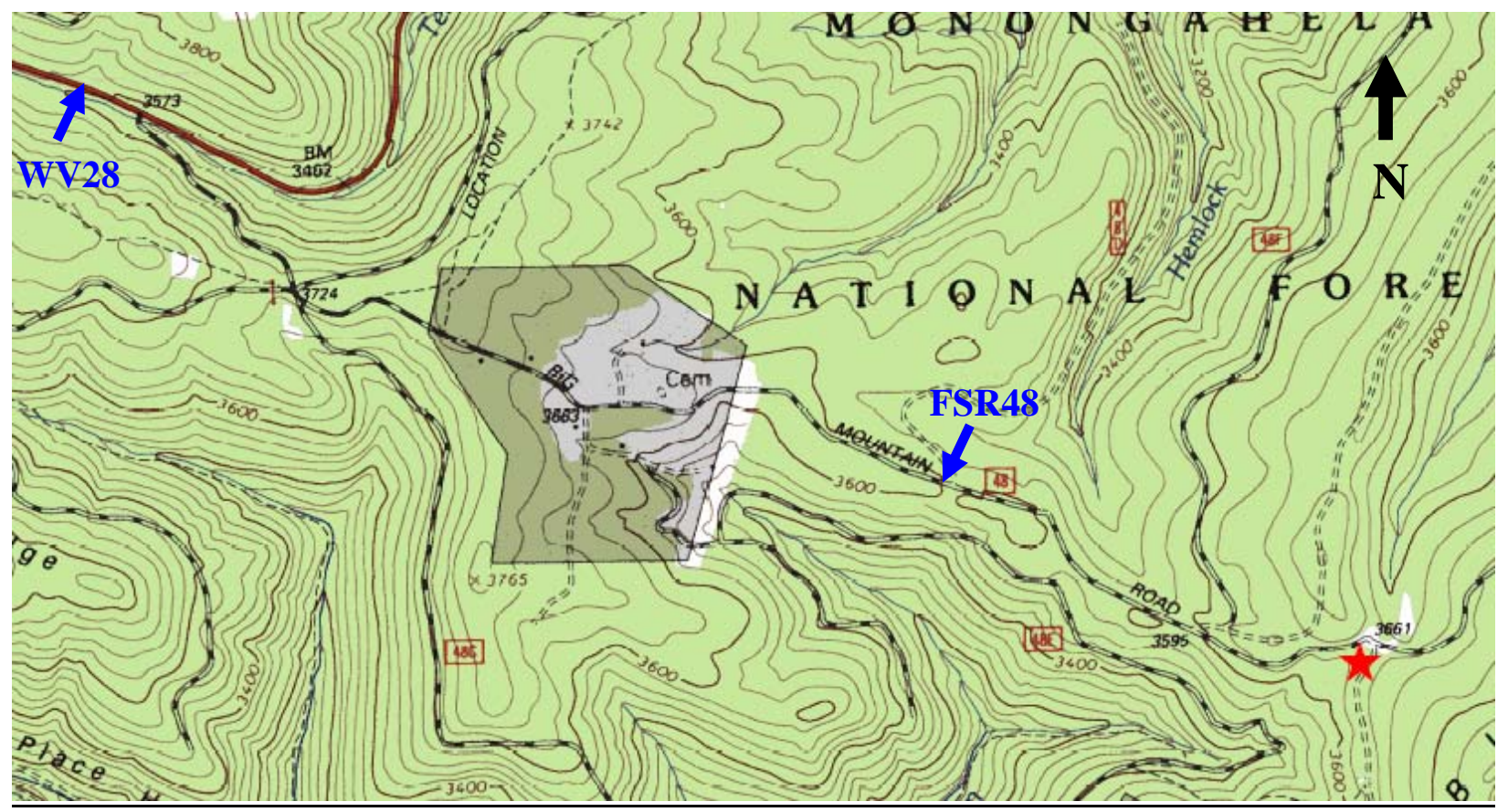

Figure 1. Topographic map of study location. The map is a portion of the Snowy Mountain USGS quadrangle map $(1: 24,000)$. The red star denotes the location of the study. WV28=WV Route 28; FSR48=Forest Service Road 48.

\section{Experimental Design and Treatments}

The study area contained 12 plots with 4 plots assigned to each of the 3 plot treatments

(Fig. 2). All chestnut trees larger than $3 \mathrm{~cm}$ diameter at breast height (DBH) were marked with fluorescent flagging. Prospective study trees were either blight-free or harbored cankers that 
encompassed less than 50\% of the tree's circumference. A plot was established when 12

prospective trees were located within close proximity to one another. Each tree within a plot was assigned a number, and the plots were designated by letters (A, B, C, etc.).

Plots A, B, C, and L were used to introduce transgenic (TG) hypovirulent strains EP146/pXHE7 and EP155/pXHE7 (Fig. 3; Table 1). Plots G, H, I, and J were treated with cytoplasmic hypovirulent strains EP146/tpTE7 and EP155/tpTE7, and plots D, E, F, and K were treated with virus-free (virulent) strains EP146 and EP155 (Fig. 3; Table 1). Each set of four plots receiving the same treatments were adjacent to one another to reduce the possibility of cross contamination of inoculum among plots (Fig. 2).

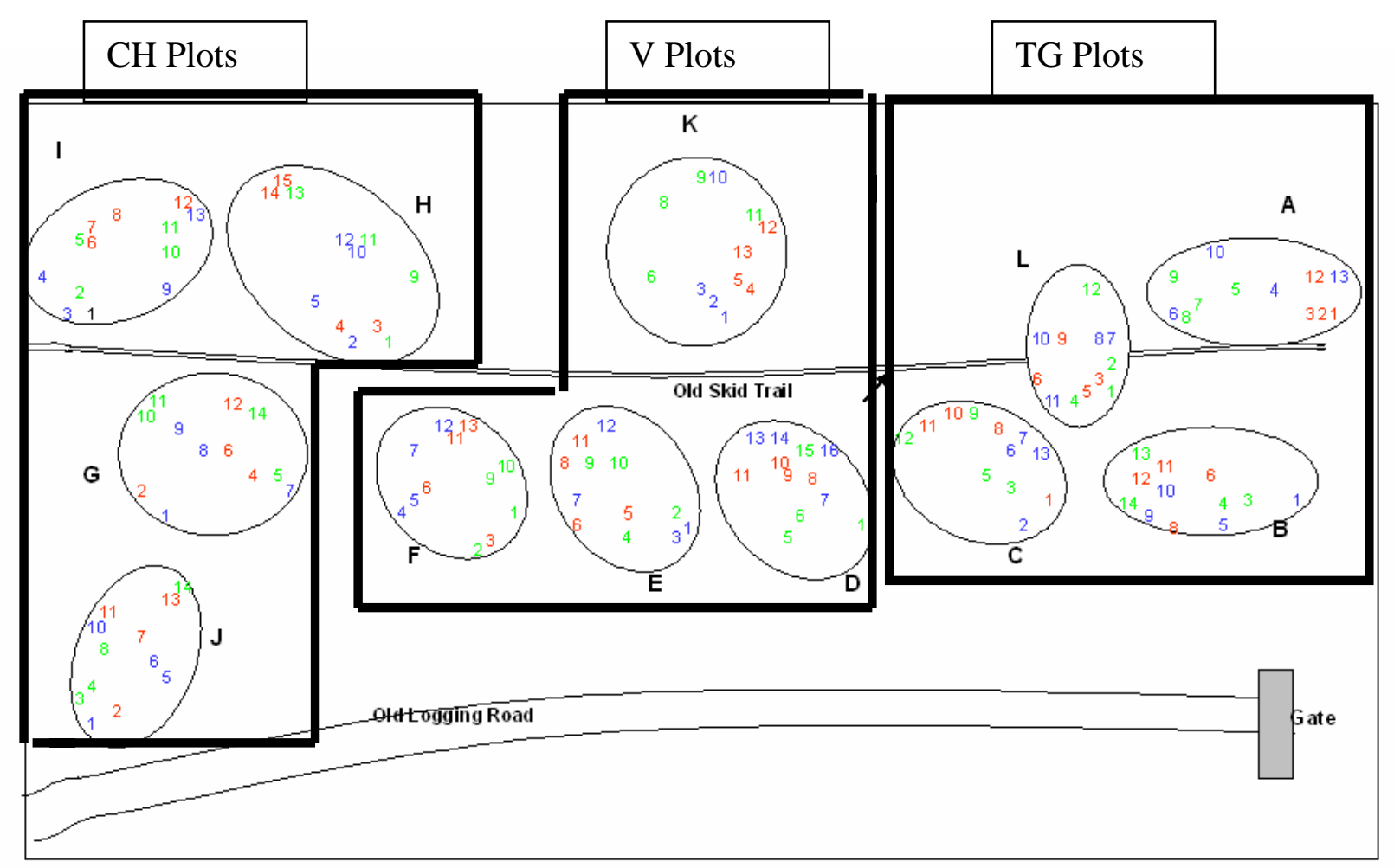

Figure 2. Study site layout. Each plot contains 12 test trees equally distributed to one of the three subtreatments designated by color (see below). Numbers represent the assigned tree number and colors represent subtreatment. Blue $=$ Scratch-initiated trees; Red = PI trees; and Green = Trap trees. 


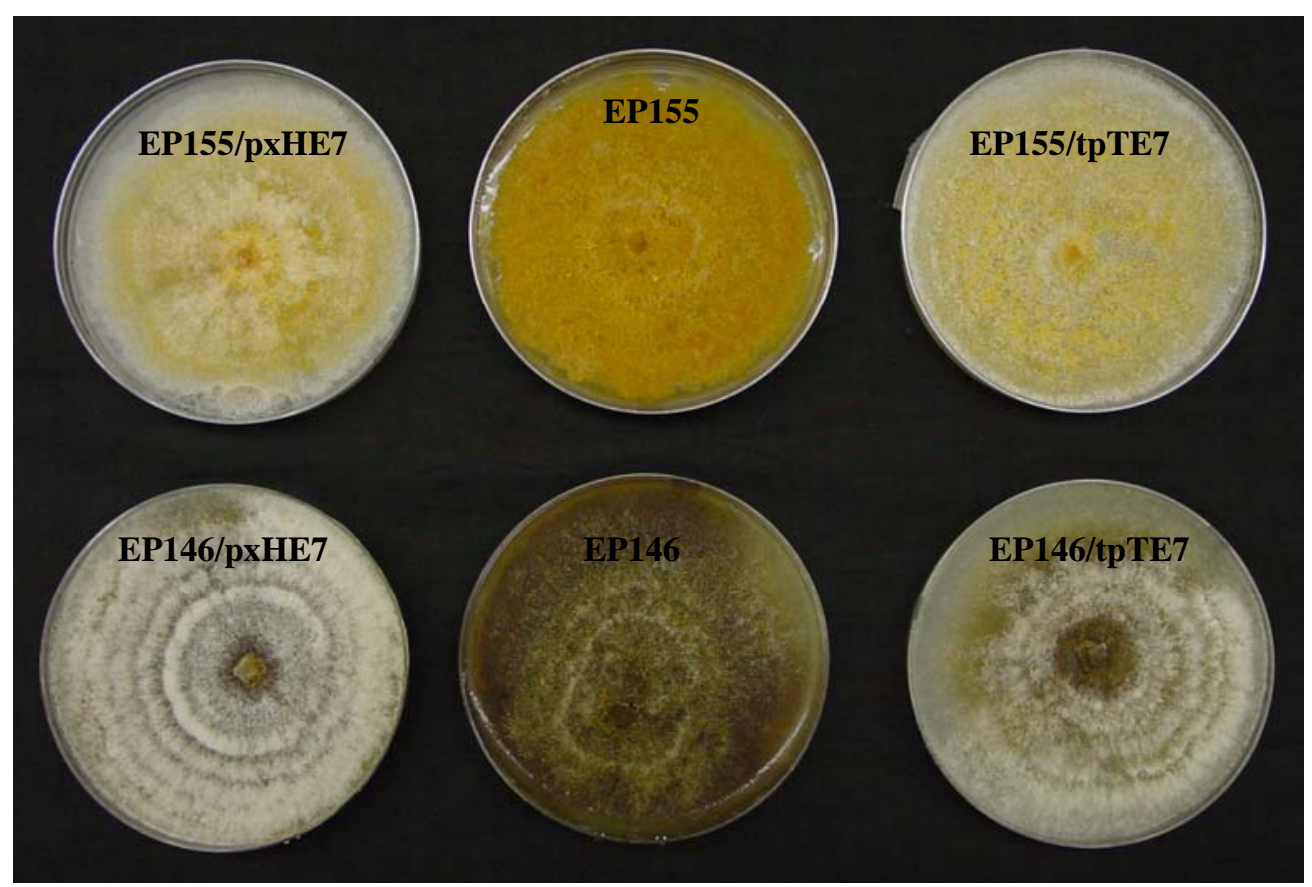

Figure 3. Cryphonectria parasitica strains that were released in the field study (see Table 1).

\section{Treatments within each plot}

Within each plot, four trees were randomly assigned to one of the following 3 subtreatments:

1.) Scratch-initiation (SI) trees - Cankers were initiated on four randomly selected trees to generate conidial inoculum for dissemination. Two cankers $(25 \mathrm{~cm} \times 6 \mathrm{~cm})$ were initiated at $0.5 \mathrm{~m}$ and $1.5 \mathrm{~m}$ above the ground on four trees in each plot in June 2004 by scraping bark with a utility knife (Fig. 4A). Wounded areas were painted with a mycelial water-agar slurry of treatment strains (prepared as described on pg. 18). The canker then was covered with an absorbent pad (Fisherbrand Scientific) to promote canker establishment (Fig. 4B). Absorbent pads were removed one month after canker initiation. Each canker on a tree was initiated with inoculum of opposite mating type, either of EP146 background (MAT-1) or EP155 background (MAT-2). Mating type treatment was randomly selected and adjusted so that each plot had two EP146-based and two EP155-based canker at each height $(0.5 \mathrm{~m}$ or $1.5 \mathrm{~m})$. 
Table 1. Cryphonectria parasitica isolates employed in this study. Vegetative compatibility type number is given when known (Anagnostakis, unpublished data); otherwise v-c group is presented as letters designated by Double and MacDonald, West Virginia University (unpublished data).

\begin{tabular}{|c|c|}
\hline Isolate & Information \\
\hline EP146 & $\begin{array}{l}\text { Brown-pigmented virulent, hypovirus-free strain isolated from the George } \\
\text { Washington National Forest near Franklin, West Virginia. ATCC\# 64671. v- } \\
\text { c 19. Mating type MAT-1 (Fig. 2). }\end{array}$ \\
\hline EP146/tpTE7 & $\begin{array}{l}\text { Lightly-pigmented, cytoplasmic hypovirulent strain in an EP146 background. } \\
\text { Transfected with hypovirus through electroporation by Nuss at the University } \\
\text { of Maryland Biotechnology Institute (Chen et al., 1994; Fig. 2). }\end{array}$ \\
\hline EP146/pXHE7 & $\begin{array}{l}\text { Lightly-pigmented, transgenic hypovirulent strain containing a cDNA copy of } \\
\text { hypovirus CHV1-Euro7 in genome of EP146. Constructed by Nuss at } \\
\text { University of Maryland Biotechnology Institute. Plasmid containing cDNA } \\
\text { copy of hypovirus also contains a hygromycin resistance }\left(\mathrm{Hyg}^{\mathrm{R}}\right) \text { gene to } \\
\text { allow for selection of TG isolates (Choi and Nuss, 1992; Fig. 2). }\end{array}$ \\
\hline EP155 & $\begin{array}{l}\text { Orange-pigmented virulent, hypovirus-free strain isolated in } 1977 \text { by S.L } \\
\text { Anagnostakis (Connecticut Agricultural Research Station) from an infected } \\
\text { chestnut sprout growing in Bethany, Connecticut. ATCC\# 38755. V-c } 40 . \\
\text { Mating type MAT-2 (Fig. 2). }\end{array}$ \\
\hline EP155/tpTE7 & $\begin{array}{l}\text { Lightly-pigmented, cytoplasmic hypovirulent strain in an EP155 background. } \\
\text { Transfected with hypovirus through electroporation by Nuss at University of } \\
\text { Maryland Biotechnology Institute (Chen et al., 1994; Fig. 2). }\end{array}$ \\
\hline EP155/pXHE7 & $\begin{array}{l}\text { Lightly-pigmented, transgenic hypovirulent strain containing a cDNA copy of } \\
\text { hypovirus CHV1-Euro7 in genome of EP146. Constructed by Nuss at } \\
\text { University of Maryland Biotechnology Institute. Plasmid containing cDNA } \\
\text { copy of hypovirus also contains a Hyg }{ }^{R} \text { gene to allow for selection of TG } \\
\text { isolates (Choi and Nuss, 1992; Fig. 2). }\end{array}$ \\
\hline PC5 & $\begin{array}{l}\text { Orange pigmented, virulent strain collected from study site in December, } \\
\text { 2003. V-c } 24 \text {. Mating type MAT-1. }\end{array}$ \\
\hline PC12 & $\begin{array}{l}\text { Orange pigmented, virulent strain collected from study site in December, } \\
\text { 2003. v-c O. Mating type MAT-2. }\end{array}$ \\
\hline PC17 & $\begin{array}{l}\text { Orange pigmented, virulent strain collected from study site in December, } \\
\text { 2003. V-c 29. Mating type MAT-2. }\end{array}$ \\
\hline PC23 & $\begin{array}{l}\text { Orange pigmented, virulent strain collected from study site in December, } \\
\text { 2003. v-c 49. Mating type MAT-1. }\end{array}$ \\
\hline PC27 & $\begin{array}{l}\text { Orange pigmented, virulent strain collected from study site in December, } \\
\text { 2003. v-c Nu. Mating type MAT-1. }\end{array}$ \\
\hline PC37 & $\begin{array}{l}\text { Orange pigmented, virulent strain collected from study site in December, } \\
\text { 2003. v-c } 2 \text {. Mating type MAT- } 2 \text {. }\end{array}$ \\
\hline PC48 & $\begin{array}{l}\text { Orange pigmented, virulent strain collected from study site in December, } \\
\text { 2003. v-c 31. Mating type MAT-2. }\end{array}$ \\
\hline PC53 & $\begin{array}{l}\text { Orange pigmented, virulent strain collected from study site in December, } \\
\text { 2003. v-c } 26 \text {. Mating type MAT-1. }\end{array}$ \\
\hline
\end{tabular}



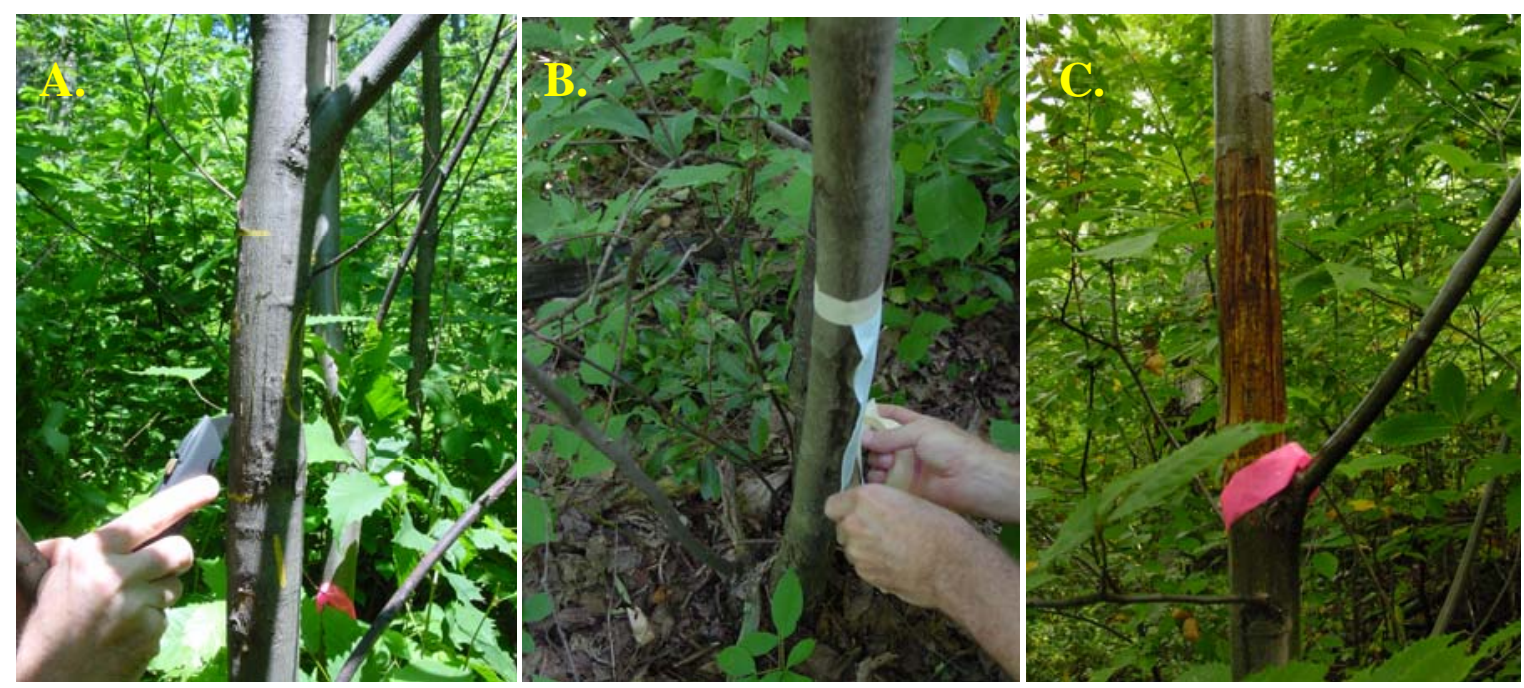

Figure 4. Techniques used to scratch-initiate cankers. A. Scratching of the bark with a sharp instrument to create wounded area. B. Covering of the wound after painting with conidial slurry. C. Established scratch initiated canker after one month of growth covered by a lab diaper.

2.) Canker treatment trees (PI Trees) - Cankers were initiated on four randomly selected trees and were spermatized with EP146 and EP155 based isolates to produce perithecia. At the beginning of the study in April, 2004, only seven natural cankers were detected on test trees. When plots lacked sufficient natural cankers, artificial infections were initiated in May, 2004. One of eight canker inciting strains (PC isolates, Table 1) were randomly assigned to a height $(0.5 \mathrm{~m}$ or $1.5 \mathrm{~m})$ on trees lacking natural infections. The eight strains used were collected in a field survey (described on page 20) and represented the eight most common v-c types so that common vic alleles would be incorporated into ascospore progeny. Wounds were punch-initiated (PI) with a $7 \mathrm{~mm}$ leather punch and inoculum applied as a similar-sized mycelial disc cut from an agar plate with a dissecting needle (hereafter "PI cankers”; Fig. 5A). Inoculations then were covered to avoid desiccation. To avoid cross-contamination when applying inoculum, eight separate leather punches and dissecting needles were used (one for each strain). When inoculations were complete, each PI tree had two cankers, whether naturally or artificially 
initiated (Fig 5B). These cankers, as well as any natural cankers that arose on these trees, were treated by painting with a conidial agar slurry in June, July, and August of 2004 in an effort to spermatize the canker inciting strain (Fig. 5C). The slurry used to treat the virulent cankers contained a conidial mixture of both mating types (MAT-1 and MAT-2) to ensure spermatization regardless of the canker initiating isolate's mating type.

3.) Trap Trees - Four trees in each plot received no treatment and were intended to be "trap" trees to assess dissemination of treatment strains from tree-to-tree. The smaller trees in each plot were selected as trap trees because the larger stems were judged to be more suitable to support potential sources of HV inoculum.
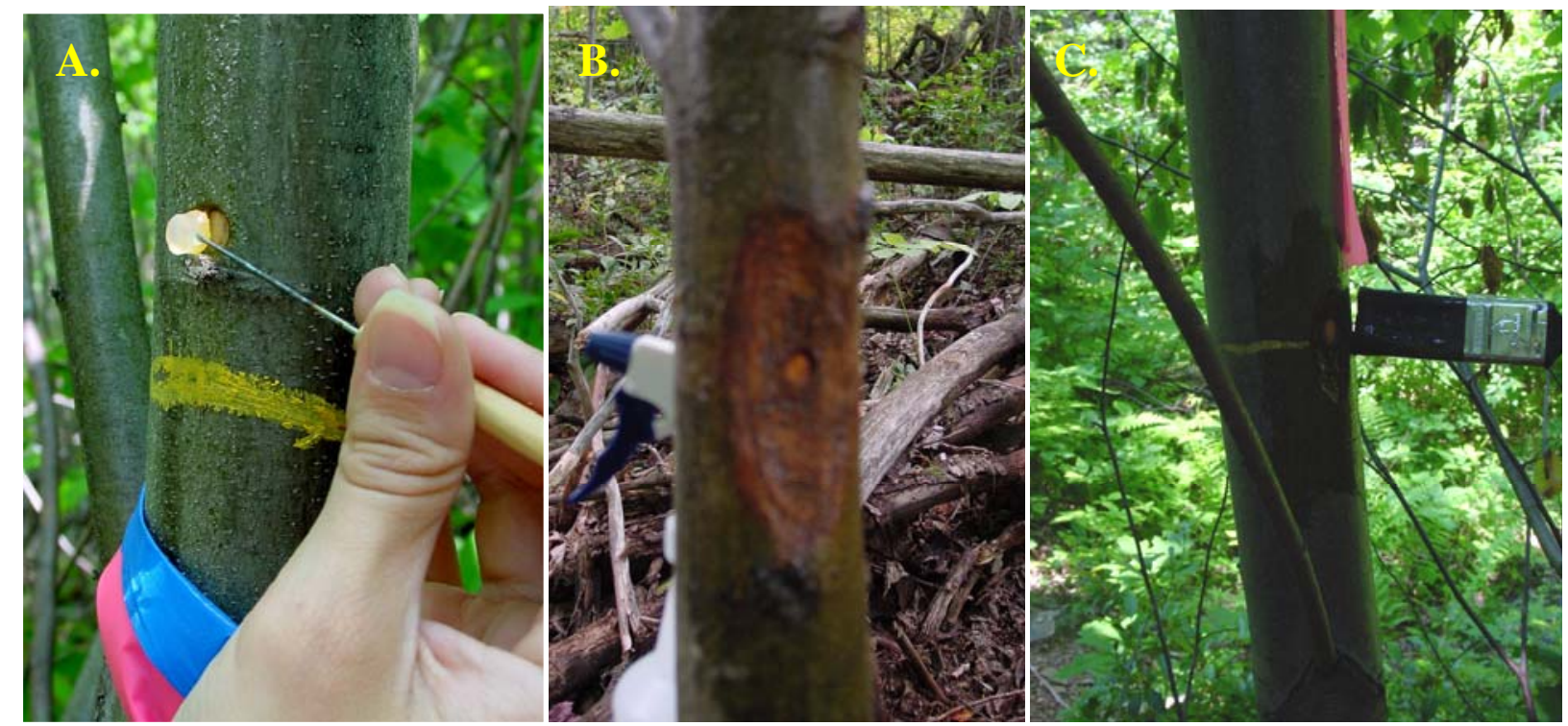

Figure 5. A. Application of mycelial disc to initiate infection after removal of bark segment with leather punch. B. Punch-initiated (PI) canker after 2 months of growth. C. Spermatization treatment being applied after development of PI canker.

\section{Preparation of Inoculum}

The inoculum used to initiate scratch-wounded cankers was delivered in a water-agar slurry. To generate this inoculum, isolates were cultured on potato dextrose agar medium 
(DIFCO) amended with $1 \mathrm{ml}$ Biotin and $0.1 \mathrm{~g}$ methionine per liter of medium (PDAmb;

Anagnostakis, 1977) and liquid glucose yeast extract (GYE; Lilly and Barnett, 1951) medium and placed under high light conditions to stimulate conidial production (Appendix A). Mycelia were collected from the liquid GYE culture by filtering through cheesecloth and blending with mycelia from 7-day-old PDAmb plates and $300 \mathrm{ml}$ of $0.1 \%$ peptone solution. Mixtures were blended with $160 \mathrm{ml}$ of $2.5 \%$ water agar to the consistency of applesauce. The blender was extensively cleaned after each inoculum preparation to avoid cross-contamination. Each slurry was placed in a plastic container and refrigerated prior to transport and application the following day at the field site.

The inoculum used to spermatize cankers also was delivered in a water agar slurry. Conidia were harvested from 12 plates of each CH and TG strain using a sterilized scalpel. Since conidia production is not impeded in virulent strains, conidia were harvested from only 3 plates of the respective V strains. Conidia were mixed with $300 \mathrm{ml}$ water containing $0.1 \%$ peptone. Spore concentrations were measured by microscopy and with the aid of a hemacytometer. Virulent, transgenic, and cytoplasmic inocula were adjusted to approximately $10^{7}$ conidia/ml. Each solution then was mixed with $200 \mathrm{ml}$ of $2.5 \%$ water agar, stored in plastic containers, and refrigerated until transportation and application.

\section{Mating Type Designation}

Mating type for each of the eight PC strains used to initiate PI cankers was determined using a procedure modified from Anagnostakis (1979). Small dormant chestnut stems were scored with a utility knife, placed in glass Petri dishes, and autoclaved. After sterilization, 4\% water agar was added until bottom of Petri dish was covered. Once the water agar solidified, mycelial plugs from a culture of one of the eight strains were transferred to dishes to inoculate 
stems. Stem pieces were spermatized after 2 and 4 weeks of growth with conidia harvested from EP146/pxHE7 or EP155/pxHE7 cultures. Four dishes were spermatized for each PC isolate: two by EP146/pxHE7 and two by EP155/pxHE7. Mating type was determined based on isolate combinations that produced perithecia. Mating type designations were later confirmed by PCR amplification of MAT specific genome regions as described by Marra (1998).

\section{Survey of vegetative compatibility diversity}

Sixty-one natural cankers were randomly sampled before the establishment of plots to determine the diversity of v-c types at the site and whether the number of v-c types could pose barriers to strain-strain interaction and hypovirus transmission. Three $2 \mathrm{~mm}$ bark plugs were removed from each canker with a bone marrow biopsy instrument (Lee-Lok Instrument Co.) and placed in individual wells of a microtiter dish (Fisherbrand Scientific; Fig. 6). The bark plugs were stored at $-20^{\circ} \mathrm{C}$ until ready for analysis.

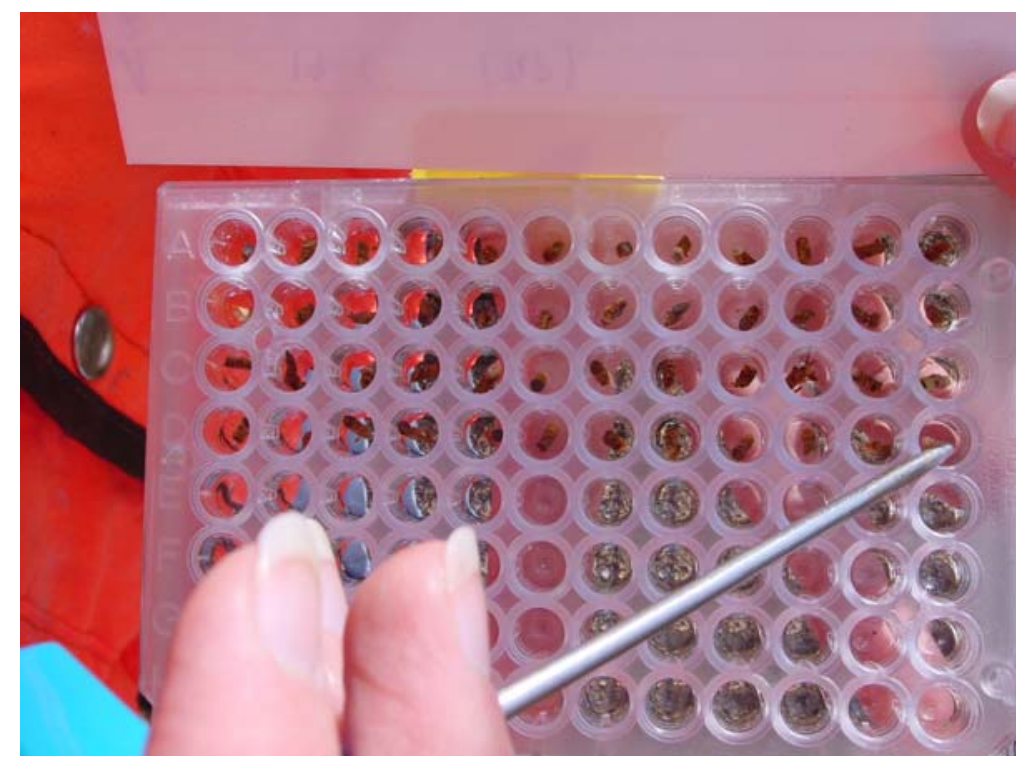

Figure 6. Collection of bark plugs in microtiter dish with bone marrow instrument.

Bark plugs were surface-sterilized by soaking in 0.5\% sodium hypochlorite solution for 14 minutes and then plated onto GYE medium amended with $10 \mathrm{mg} / \mathrm{l}$ streptomycin sulfate and 
100 mg/l chlortetracycline (GYE/A; Appendix 1). One isolate obtained from each canker was labeled PC1-PC61, transferred to PDAmb, and cultured at $20^{\circ} \mathrm{C}$ in a 16:8 photoperiod for 7-10 days. Forty-two known v-c tester isolates from the West Virginia University collection also were cultured on PDAmb under the same conditions described above. Each PC isolate was paired with each v-c tester strain, five pairings per Petri dish (Fig. 7) on Brom Cresol Green medium (Powell, 1995; Appendix 1). Vegetative incompatibility was determined by the presence of a "barrage” between incompatible isolates as described by Anagnostakis (1977) (Fig. 7). All PC isolates that were incompatible with any of the tester strains were paired amongst each other to establish other common v-c types.

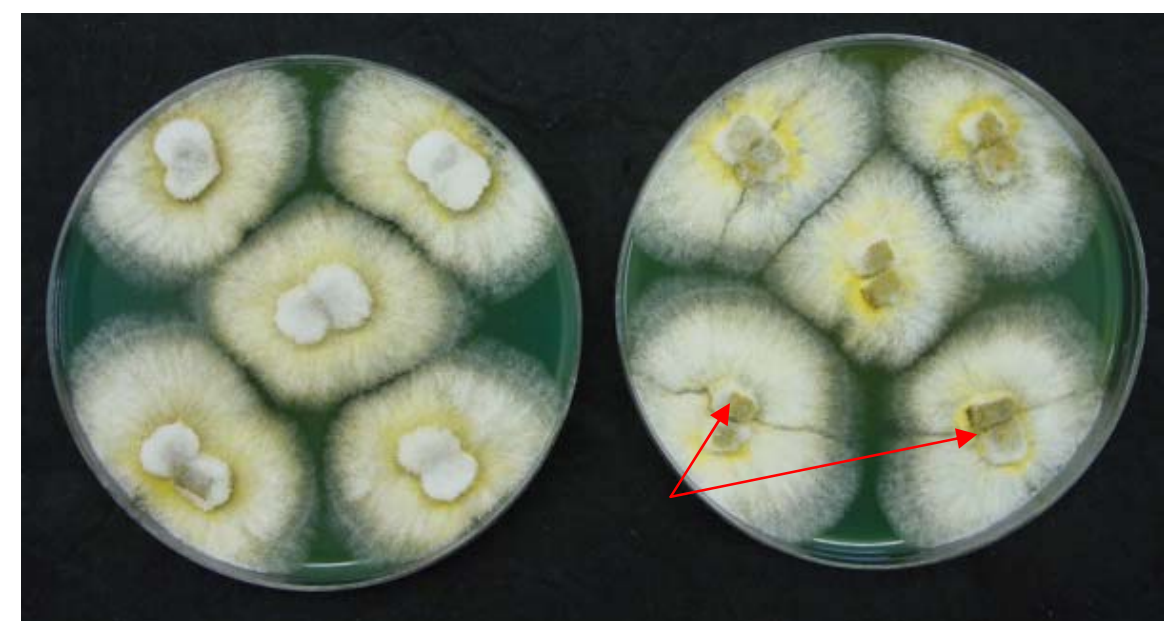

Figure 7. Vegetative compatibility testing. Presence of barrage (arrows) indicates vegetatively incompatible isolates.

\section{Tree condition}

Measurements of tree condition included: diameter of tree at $4.5 \mathrm{ft}$. above ground level (measured in spring and fall of 2004) and an overall tree health rating based on presence of chlorosis/necrosis in crown (measured in May and August, 2004 using criteria in Table 2). Tree health was subjectively rated by one person to maintain consistency. 
Table 2. Tree health scale.

\begin{tabular}{|c|c|}
\hline Rating & Criteria \\
\hline $\mathbf{0}$ & Completely dead above root collar. \\
\hline $\mathbf{1}$ & Severe chlorosis/necrosis in crown. Portions of crown living. \\
\hline $\mathbf{2}$ & Slight chlorosis/necrosis in crown. \\
\hline $\mathbf{3}$ & No chlorosis/necrosis present in crown. \\
\hline
\end{tabular}

\section{Development of Artificial and Natural Infections}

Establishment of SI cankers was assessed by measuring the increase in area beyond the initial wounded area. Severity of natural and PI infections was assessed using a canker rating scale based on the presence of fungal stroma and tree callus tissue (Table 3).

Table 3. Canker rating scale.

\begin{tabular}{|c|c|}
\hline Rating & Criteria \\
\hline $\mathbf{1}$ & No stroma. Heavy callus tissue. \\
\hline $\mathbf{2}$ & $\begin{array}{c}\text { Slight amount of stroma. Moderate } \\
\text { callus tissue present. }\end{array}$ \\
\hline $\mathbf{3}$ & $\begin{array}{c}\text { Moderate - heavy stroma. Limited callus } \\
\text { tissue present }\end{array}$ \\
\hline $\mathbf{4}$ & Heavy stroma. No callus tissue. \\
\hline
\end{tabular}

\section{Sampling Artificial and Natural Cankers}

Natural and PI cankers were sampled to assess virus infection status of each thallus when first discovered and in October, 2004. Hypovirus status of cankers was evaluated by collecting six $2 \mathrm{~mm}$ bark plugs per canker with a bone marrow biopsy instrument and storing them in labeled microtiter dishes as described for v-c type diversity survey (Fig. 6). Bark plugs were surface-sterilized by soaking in $0.5 \%$ sodium hypochlorite solution for 14 minutes and then plated onto GYE/A. Resulting isolates (Fig. 8A) were transferred to PDAmb and cultured at $20^{\circ}$ C in a 16:8 photoperiod for 7-10 days and then assessed for their hypovirus infection status based 
on culture morphology and pigmentation (Fig. 8B). Isolates showing hypovirulent

characteristics were transferred to PDAmb containing hygromycin (40 $\mu \mathrm{g} / \mathrm{ml})$ to determine the presence of the transgenic insert. A representative sample of virulent isolates from all three main treatments also was tested for hygromycin resistance (hyg ${ }^{\mathrm{R}}$ ).

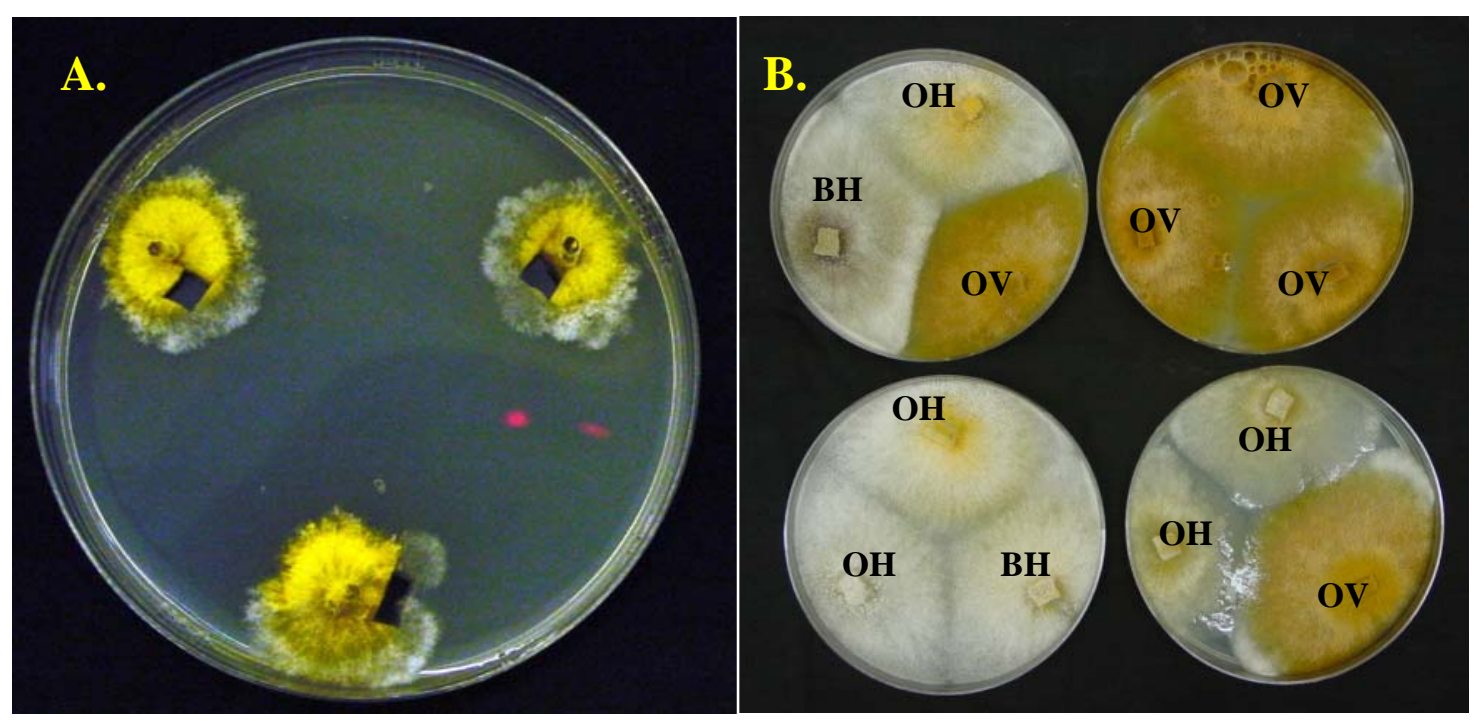

Figure 8. Procedure for isolating C. parasitica from bark plugs. A. Colonies growing from surface-sterilized bark plugs on GYE/A. B. Subcultured colonies growing on PDAmb. These colonies are labeled as they were scored: $\mathrm{OV}=$ orange-virulent; $\mathrm{OH}=$ orange-hypovirulent; $\mathrm{BH}=$ brown-hypovirulent; $\mathrm{BV}=$ brown-virulent (not shown).

\section{Ascospore sampling}

In October 2004, bark discs were collected from all PI and natural cankers in the TG plots and from a representative number of the same types of cankers in V and $\mathrm{CH}$ plots. Perithecia were sampled from bark discs in order to isolate individual ascospore colonies and score them for hypovirus infection status. Bark discs were removed from cankers with a 7mm leather punch and placed in labeled tissue-culture dishes (Fisherbrand Scientific) and stored at $20^{\circ} \mathrm{C}$ (Fig. 9). When ready for processing, bark discs were placed in a glass Petri dish containing warm water to promote thawing. Perithecia were teased from stroma (Fig. 10A). 
Extraneous stromatal debris also was removed from the surface of a perithecium by using a dissecting needle and by rolling the perithecium on the surface of $4 \%$ water agar. The perithecium then was transferred to a watch glass containing $2 \mathrm{ml} 0.1 \%$ peptone solution (hereafter "peptone") and ascospore contents were liberated using a dissecting needle (Fig. 10B). The ascospore solution then was placed into a $1.5 \mathrm{ml}$ microcentrifuge tube, vortexed, and $0.5 \mathrm{ml}$ was transferred to a PDAmb plate. One-half $\mathrm{ml}$ of the solution was further diluted in a microcentrifuge tube containing $1 \mathrm{ml}$ peptone, vortexed, and transferred to a PDAmb plate. This dilution ensured suitable distribution of ascospores to allow for selection of single colonies when spores germinated. After 24 hours, single germinating ascospores were transferred to PDAmb (five/plate) and cultured at $20^{\circ} \mathrm{C}$ for 7-10 days in 16:8 photoperiod and then evaluated for their hypovirus infection status based on culture morphology and pigmentation (Fig. 10C). Five perithecia per canker were sampled and 25 ascospores per perithecium were cultured. A representative number of isolates (both virulent and hypovirulent) then were transferred to PDA containing hygromycin to determine the presence of the $\mathrm{hyg}^{\mathrm{R}}$ gene representative of the transgenic strain. 


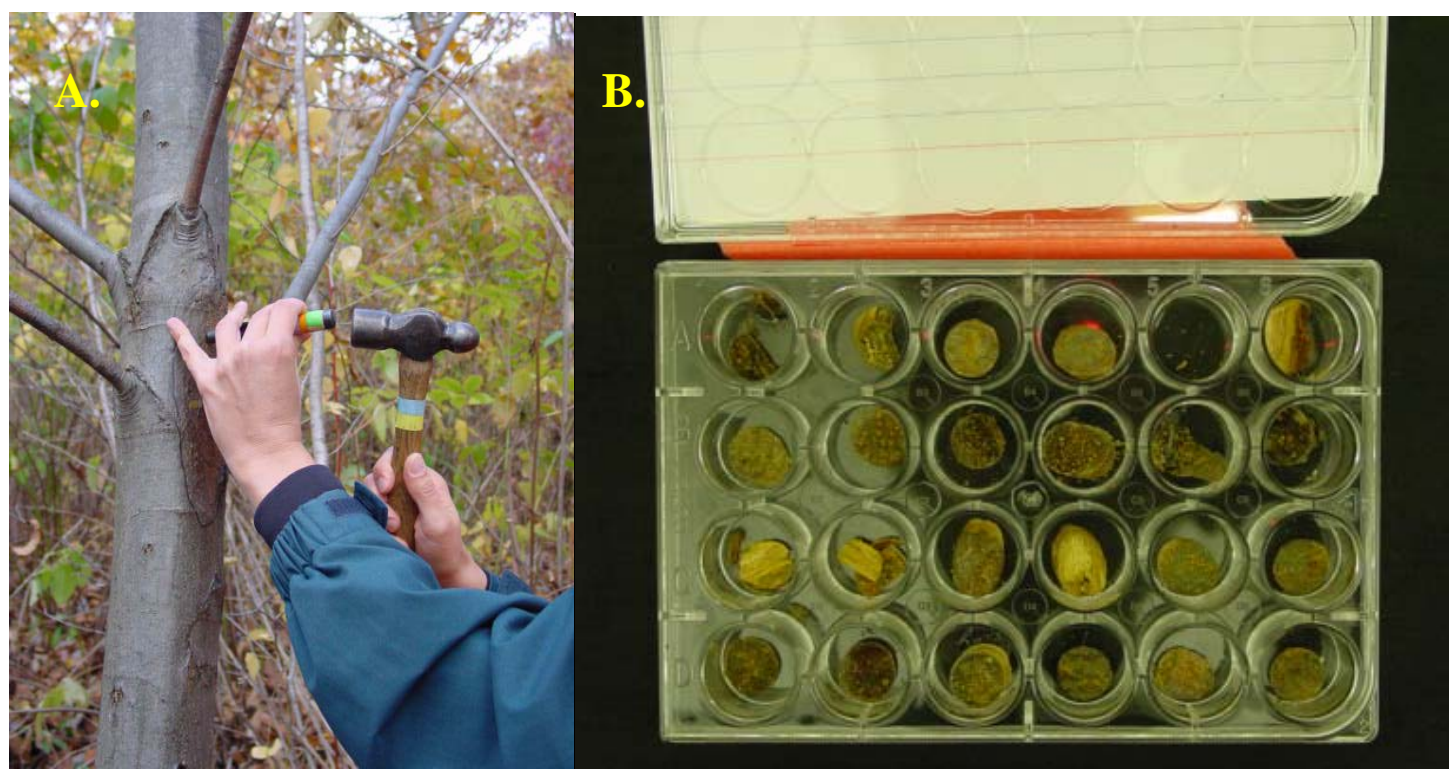

Figure 9. A. Use of a leather punch to remove bark discs from canker. B. Storage of bark discs in a labeled tissue culture dish.
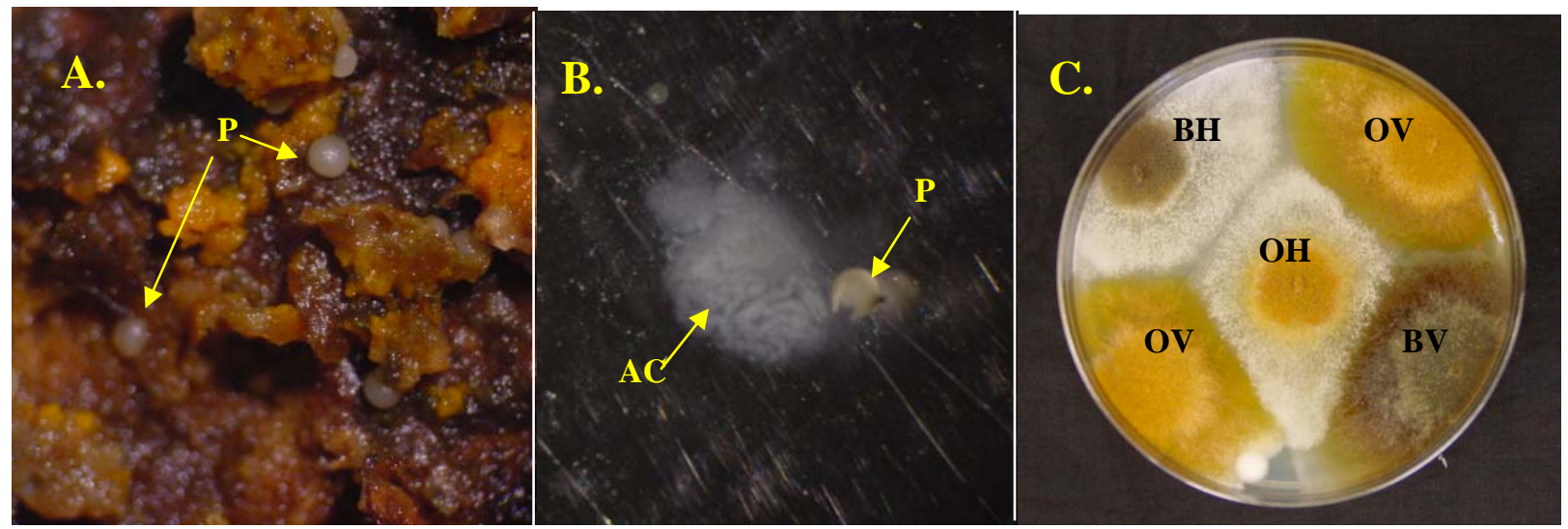

Figure 10. A. Removal of perithecia from stroma on a bark disc. B. Squashed perithecium to liberate ascospores. C. Five colonies resulting from the selection of a five germinating ascospores and each scored for their morphology (see Fig. 7 for abbr.). P=perithecia; AC=ascospore contents.

\section{Conidia Sampling}

Conidia also were collected from a representative number of scratch-initiated cankers.

Conidia were isolated by serially diluting the contents of pycnidia in a fashion similar to aforementioned description for perithecia. Some conidia also were sampled in vitro by harvesting conidia from the surface of $C$. parasitica cultures on PDAmb and serially diluting in peptone solution. After 48 hours, conidia were harvested and plated onto PDAmb and cultured 
at $20^{\circ} \mathrm{C}$ for $7-10$ days in a 16:8 photoperiod. Resulting colonies were examined for hypovirulent characteristics. A representative number of conidial isolates then were transferred to PDA containing hygromycin to determine whether they carried the hyg ${ }^{\mathrm{R}}$ gene.

\section{Double-stranded RNA Extraction and Pathogenicity Testing}

Presence of dsRNA was determined for isolates displaying unexpected morphologies. Isolates to be tested were grown on cellophane (Flexal Corporation) overlaid on GYE/A for 1014 days in a 16:8 photoperiod. Mycelia were harvested and ground to powder using mortar and liquid nitrogen. Ground samples were placed in scintillation vials and stored at $-20^{\circ} \mathrm{C}$ until analysis. Double-stranded RNA then was purified from mycelial samples using a procedure modified from Morris and Dodds (1979; Appendix B). Fragments of dsRNA were discerned using gel electrophoresis (2 hours at 100V) in 1.0\% agarose gel stained with ethidium bromide. Resulting bands were examined under ultra-violet light and photographed using Genesnap software (Syngene).

Pathogenicity of certain atypical isolates was quantified using a modified apple pathogenicity test (Fulbright, 1984). Three positions were assigned on each apple and each isolate of interest was randomly assigned to a position. The diameters of resulting lesions were measured one, two, and three weeks after inoculation.

\section{Evaluation of the Potential for hypovirus transfer to other v-c types}

Strains EP146/pXHE7 and EP155/pXHE7 were paired five times against all v-c types collected from the site to evaluate the ability of the transgenic treatment strains to transmit their hypovirus to resident $C$. parasitica strains. Transmission of the cDNA copy of the hypovirus to other v-c types was measured by pairing these non-converted v-c types from the study site with hypovirulent/hyg ${ }^{\mathrm{R}}$ ascospore progeny obtained from perithecia. Pairings were conducted in 
rounds. During each round, three hypovirulent/hyg ${ }^{\mathrm{R}}$ ascospore isolates were paired five times with v-c types unable to be converted by parental strains. The pairings then were cultured on PDA and incubated in a 16:8 photoperiod for 12-14 days and examined for evidence of hypovirus transmission (“conversion”; Fig. 11). When a v-c type acquired hypovirus four or five times, it was dropped from further analysis. The objective of this analysis was to observe dissemination of the cDNA transgene to other v-c types. By showing virus acquisition by many v-c types that were unable to be converted by the parental strains, the greater disseminative potential of transgenic strains will be illustrated.

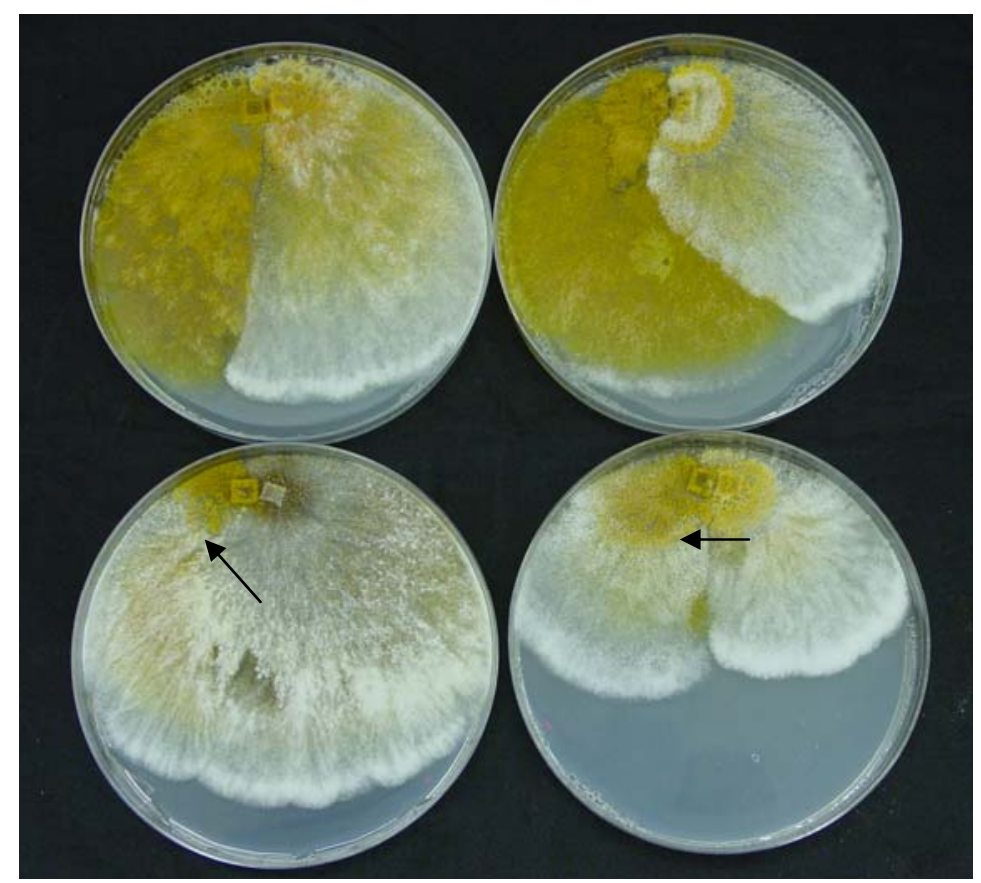

Figure 11. Non-converted (above) and converted (below) isolates. Virulent strains were placed on the left side of each plate and hypovirulent strains on the right side. Arrows indicate the point where virulent strains start displaying hypovirulent morphology.

\section{Statistical Analyses}

Three treatments (TG, CH, V) allowed for comparison of transgenic strains containing a nuclear copy of the hypovirus (TG plots) to strains of the same background (EP146/EP155) 
containing either cytoplasmic copies of the hypovirus ( $\mathrm{CH}$ plots) or no hypovirus (V plots). Analysis of Variance (ANOVA) procedures were used in a split plot design with TG, V, and CH being the main plot treatments. As can be seen in Figure 1, main plot treatments were grouped to avoid encroachment of other main plot treatment propagules. These statistical analyses were run assuming each main plot treatment was randomly assigned to one of the 12 plots; otherwise there would be no replication to test main treatment effects. Results were considered significant if probability levels were less than 0.05 .

Analysis of Variance procedures in split-plot design (sub-treatment = PI, SI, Trap trees) were used to compare percent change in DBH and tree health ratings. Increase in area of SI cankers was analyzed using ANOVA in split-plot design with strains EP146 and EP155 (and their isogenic/hypovirulent counterparts) as sub-treatments. Canker ratings were analyzed with ANOVA in split-plot design with natural vs. initiated and painted vs. non-painted as sub treatments. Analysis of variance tables from these analyses are listed in Appendix C. Differences were discerned using Scheffe’s comparison test at a p-value of 0.05. The objective of these analyses was to detect differences that may indicate varying effects on tree health among the main treatments. Analysis of Variance procedures in a completely random design also were used to compare lesion diameters among isolates in the apple pathogenicity test.

A chi-squared goodness-of-fit test was used to compare hypovirus recovery rates of spermatized cankers in TG plots to those in $\mathrm{CH}$ plots. This analysis was used to illustrate any difference in conversion potential of either strain.

Inheritance of pigmentation, hyg ${ }^{\mathrm{R}}$, and the transgene by ascospores were compared among the three main treatments. A chi-squared goodness-of-fit test was used to compare the 
viral cDNA inheritance rate of outcrossed perithecia to an expected Mendelian ratio of 1:1:1:1 (OV:BV:OH:BH). 


\section{$\underline{\text { Results }}$}

\section{Survey of vegetative compatibility diversity}

Before plots and study trees were delineated, 61 cankers were sampled to establish a measure of the v-c type diversity at the site. A total of 29 different v-c types were identified from extant cankers at the site. Nineteen of the types were in common with v-c test strains in the West Virginia collection. Ten additional types were identified after pairing the undesignated isolates with each other to delineate the remaining v-c types. The 10 additional types were designated “AA, BB...etc" in the order they were discovered (Table 4). The most common v-c types were D, F, K, and L.

Table 4. Canker distribution of different v-c types.

\begin{tabular}{|c|c|c|c|}
\hline $\begin{array}{l}\text { V-c } \\
\text { Group }\end{array}$ & $\begin{array}{c}\text { \# of } \\
\text { Cankers }\end{array}$ & $\begin{array}{l}\text { v-c Group } \\
\text { (continued) }\end{array}$ & $\begin{array}{c}\text { \# of } \\
\text { Cankers }\end{array}$ \\
\hline$A$ & 1 & Delta & 1 \\
\hline $\mathrm{D}$ & 7 & Epsilon & 1 \\
\hline$E$ & 1 & $\mathrm{Nu}$ & 2 \\
\hline$F$ & 6 & Kappa & 1 \\
\hline G & 2 & AA & 2 \\
\hline $\mathrm{H}$ & 3 & BB & 4 \\
\hline K & 5 & $\mathrm{CC}$ & 2 \\
\hline$L$ & 5 & DD & 2 \\
\hline$M$ & 1 & EE & 1 \\
\hline $\mathrm{N}$ & 1 & FF & 1 \\
\hline $\mathrm{O}$ & 4 & GG & 1 \\
\hline $\mathrm{P}$ & 1 & $\mathrm{HH}$ & 1 \\
\hline $\bar{Q}$ & 1 & II & 1 \\
\hline$T$ & 1 & $\mathrm{JJ}$ & 1 \\
\hline W & 1 & & \\
\hline
\end{tabular}

\section{Tree Condition}

Diameter at breast height (DBH) was analyzed for 144 trees (48 in each plot treatment and 16 for each sub-treatment). There were no significant differences between the average percent increase in $\mathrm{DBH}$ of plot treatments $(\mathrm{P}>\mathrm{F}=0.16)$ or sub-treatments $(\mathrm{P}>\mathrm{F}=0.29)$ during the 2004 growing season. Trees in virulent plots showed the greatest percent increase in DBH. 
A subjective tree health rating was assigned to all 144 trees in May and August, 2004. Most trees were rated as healthy in May (before treatment application) and August (Table 5); a few noted exceptions were when mortality occurred above natural cankers. No significant changes in tree health rating were detected among plot treatments $(\mathrm{P}>\mathrm{F}=0.69)$ or sub-treatments $(\mathrm{P}>\mathrm{F}=0.69)$.

Table 5. Average tree health rating ${ }^{1}$ in May and August, 2004.

\begin{tabular}{ccccccc}
\hline \multirow{2}{*}{ Treatment tree } & \multicolumn{2}{c}{ Transgenic } & \multicolumn{2}{c}{ Virulent } & \multicolumn{2}{c}{ Cytoplasmic } \\
& May & August & May & August & May & August \\
\hline PI & 2.88 & 3.00 & 3.00 & 2.88 & 2.88 & 2.88 \\
SI & 2.94 & 2.88 & 3.00 & 3.00 & 3.00 & 2.88 \\
Trap & 2.94 & 2.88 & 3.00 & 3.00 & 3.00 & 2.94 \\
\hline
\end{tabular}

1 Tree health rating criteria given in Table 2, Materials and Methods

\section{Development of Artificial and Natural Infections}

Twelve natural cankers were discovered on study trees when they were established in April, 2004. After the first growing season, 48 additional natural infections were recorded. Punch-initiated (PI) trees harbored the most natural infections in each plot type except in the transgenic (TG) plot, in which scratch-initiated (SI) trees harbored more cankers (Table 6). The number of cankers found on trap trees was low in each treatment (Table 6).

Table 6. Number of natural cankers discovered in May and October of the 2004 field season.

\begin{tabular}{ccccccc}
\hline $\begin{array}{c}\text { Treatment } \\
\text { tree }\end{array}$ & \multicolumn{2}{c}{ Transgenic } & \multicolumn{2}{c}{ Virulent } & \multicolumn{2}{c}{ Cytoplasmic } \\
May & October & May & October & May & October \\
\hline PI & 3 & 10 & 2 & 12 & 3 & 7 \\
SI & 3 & 12 & 0 & 3 & 0 & 3 \\
Trap & 1 & 5 & 0 & 5 & 0 & 3 \\
\hline
\end{tabular}

The scratch method used to initiate cankers appeared effective when the scratched areas were initially unwrapped in July, 2004 (Fig. 12A), but by October the bark of several SI cankers exfoliated (Fig. 12B) and stroma production appeared low. The length and width of SI cankers 
were used as a measure of SI canker establishment. A total of 96 SI cankers were measured across all plot types. Cankers initiated with virulent strains EP146 and EP155 showed a significantly larger increase in area than either the transgenic or cytoplasmic strains $(\mathrm{P}>\mathrm{F}=.02)$ (Fig. 13). Even though strain EP155 produced larger increases in canker area than EP146, the difference was marginally non-significant $(\mathrm{P}>\mathrm{F}=0.06)$ (Fig 13).

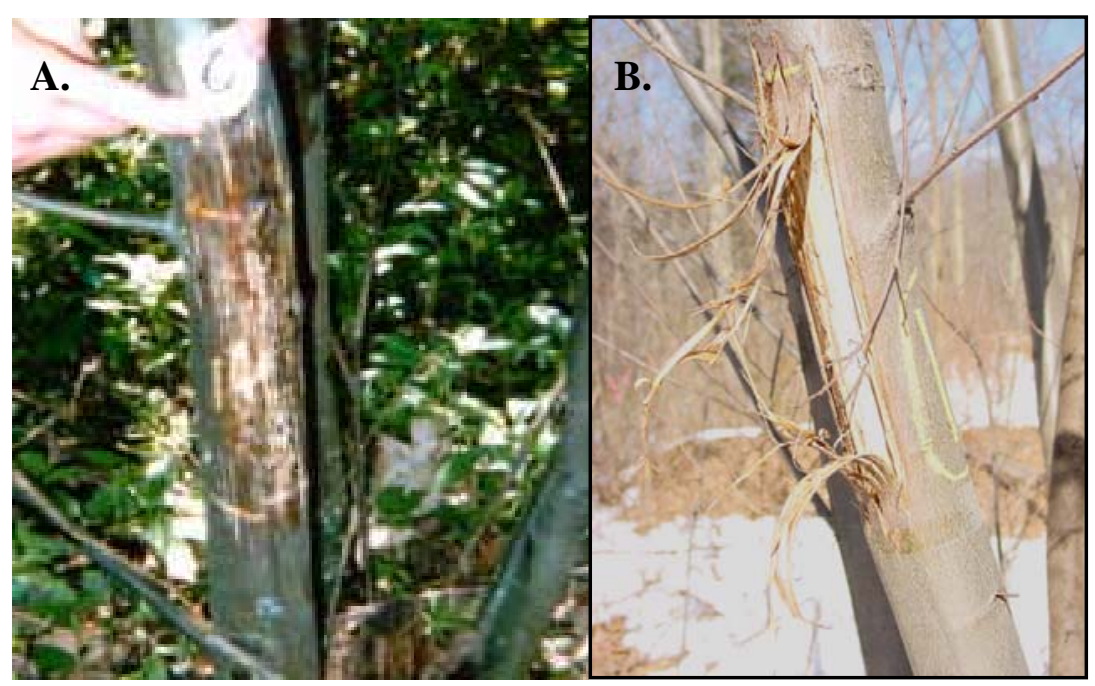

Figure 12. A. Stroma evident on SI canker in a $\mathrm{CH}$ plot one month following initiation. B. Bark exfoliation of SI canker in a TG plot.

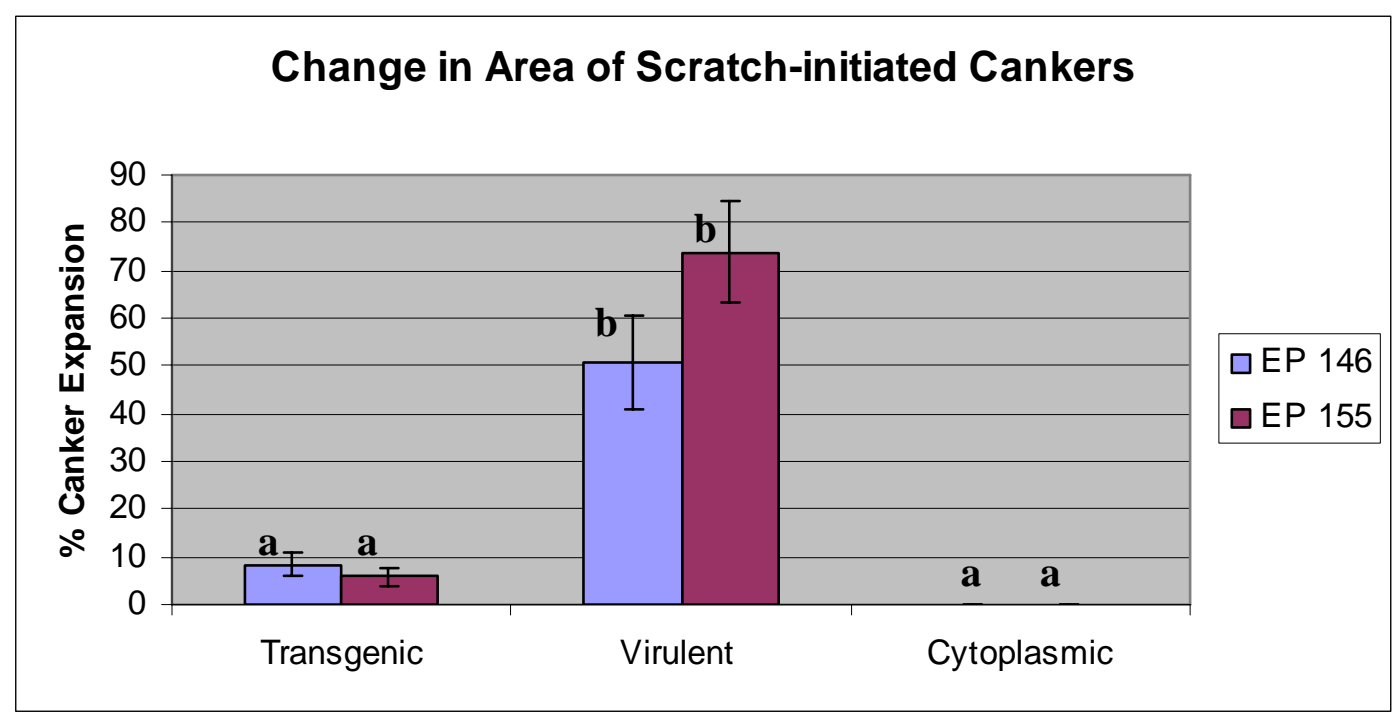

Figure 13. Expansion of scratch-initiated cankers. Error bars show +/- 1 SE. Differing letters represent significant differences. 
Natural and PI cankers were subjectively rated based on presence of tree callus and fungal stroma (Table 3); cankers showing the most callus and least stroma rated the highest. A total of 148 cankers $(56=\mathrm{TG}, 42=\mathrm{CH}, 50=\mathrm{V})$ were rated in October, 2004. Overall average ratings indicate that cankers from the cytoplasmic plots appeared significantly less virulent compared to those in the virulent and transgenic plots $(\mathrm{P}>\mathrm{F}=0.0032)$. Natural cankers had significantly higher average ratings $(\mathrm{P}>\mathrm{F}=0.0091)$ when compared to $\mathrm{PI}$ cankers in each of the three treatments (Fig 14). There was no significant relationship between cankers that received treatment inoculum versus those that did not $(\mathrm{P}>\mathrm{F}=0.9625$; Fig 15).

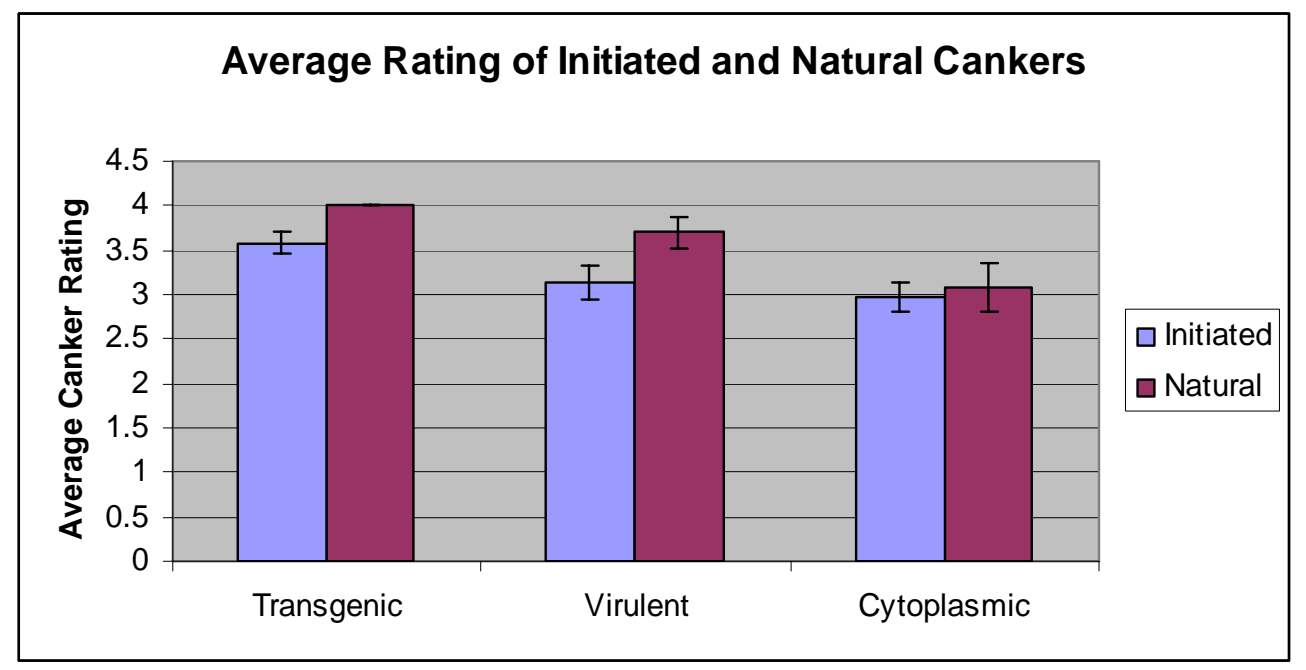

Figure 14. Average canker ratings of initiated and natural cankers in October, 2004. Error bars show +/- 1 SE.

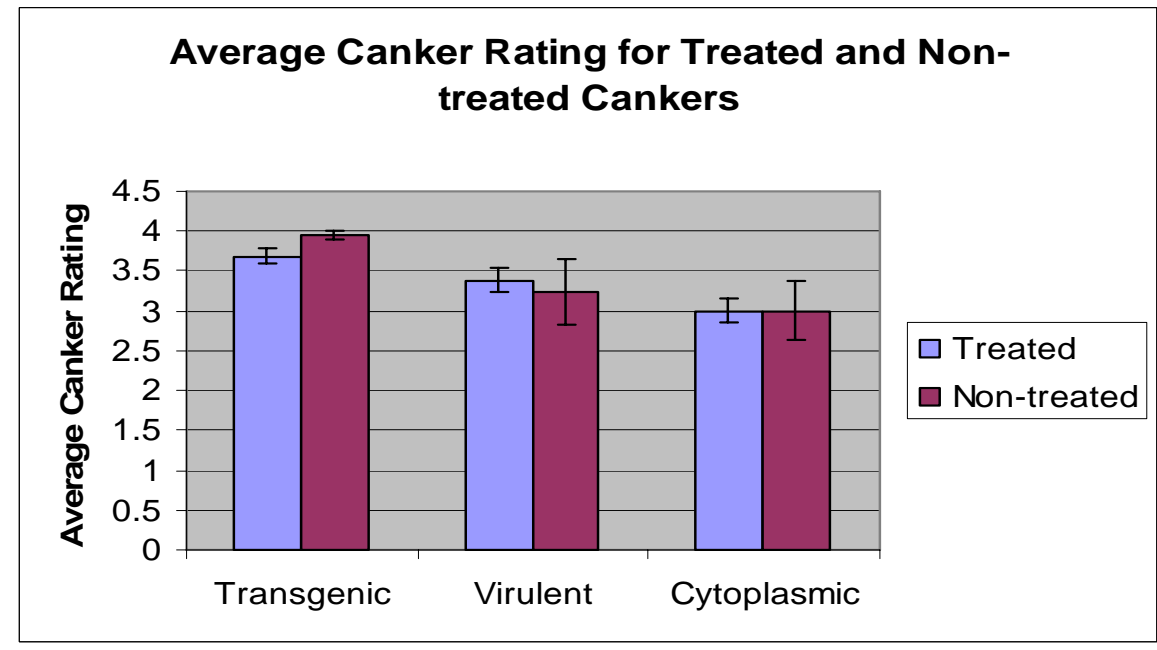

Figure 15. Average canker rating for treated and non-treated cankers in October, 2004. Error bars show +/- 1 SE. 
There also was no obvious trend between canker rating and number of HV isolates recovered from a canker. Figure 16 shows the average rating of cankers from each of the six HV recovery classes (recovery classes represent the number of $\mathrm{HV}$ isolates recovered from a canker: “0/6” $=0$ plugs out of 6 were HV while " $6 / 6 ”=6$ plugs out of 6 were HV). The graph is heavily weighted because there were varying numbers of cankers in each recovery class (Fig. 16). Treated cankers were the most likely to yield HV isolates (see next section).

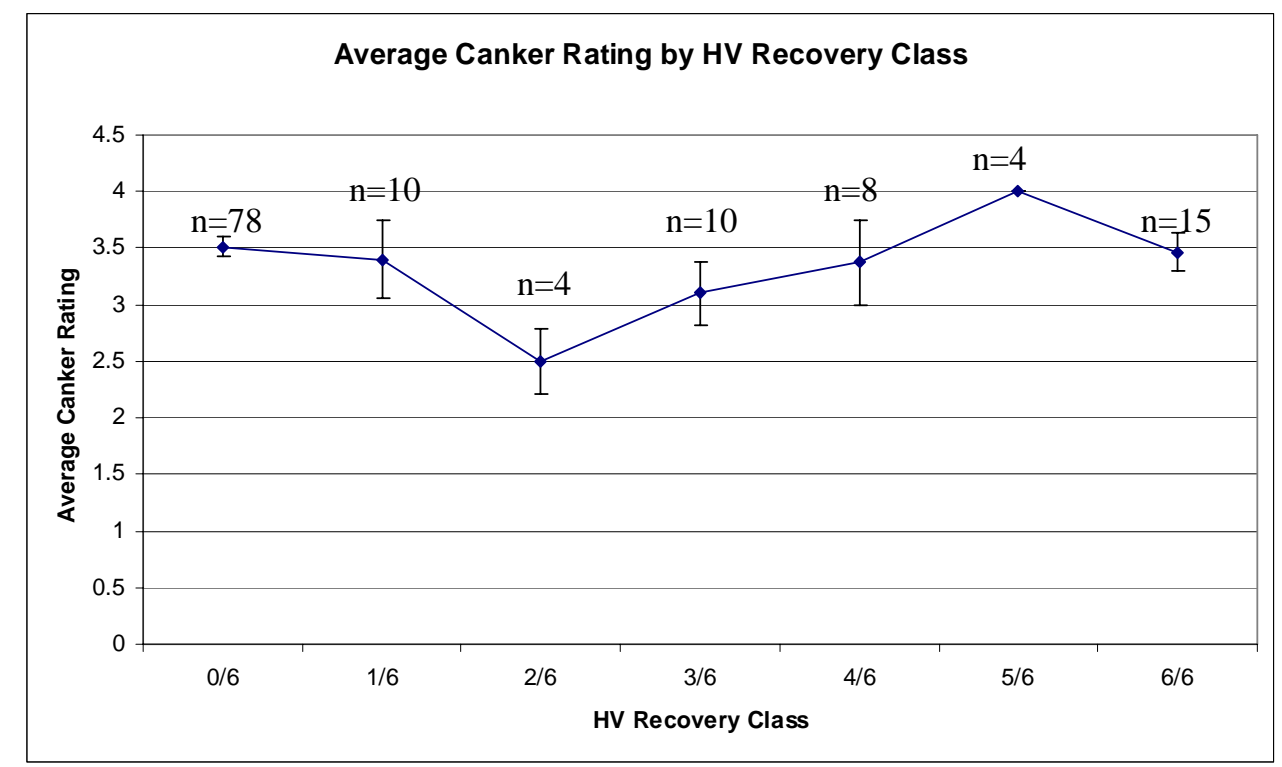

Figure 16. Average rating of cankers from different HV recovery classes. Data set includes cankers from both TG and $\mathrm{CH}$ plots. Error bars show +/- 1 SE. " $\mathrm{n}$ " is the number of cankers in each class.

\section{Sampling Artificial and Natural Cankers}

Six bark plugs were collected from each of 129 natural and artificially established cankers ( $53=\mathrm{TG}, 34=\mathrm{V}, 42=\mathrm{CH}$ ) across the three plot treatments. Each plug was plated on growth medium and the resulting colonies were subcultured and scored for culture morphology (Fig. 8). The majority of isolates recovered from each canker type displayed the orange-virulent 
(OV) morphology. The highest percentages of $\mathrm{HV}$ isolates were collected from cankers in both the TG and CH plots that had been treated with HV spermatia ("PI" and "NatPI"; Fig. 17).

Treated cankers from TG and CH plots yielded similar distributions of morphology types when analyzed for C. parasitica isolates $\left(\mathrm{P}>\chi^{2}=0.251\right.$; Fig. 17). When contaminants were included in the analysis, the distributions were not similar $\left(\mathrm{P}>\chi^{2}=<0.001\right)$. Hypovirulent isolates also were recovered from natural cankers on SI trees in all three plot treatments. There was no HV recovery from natural cankers on trap trees in TG or $\mathrm{CH}$ plots, though three bark plugs isolated from one trap tree in a V plot displayed HV morphology (Fig. 17).

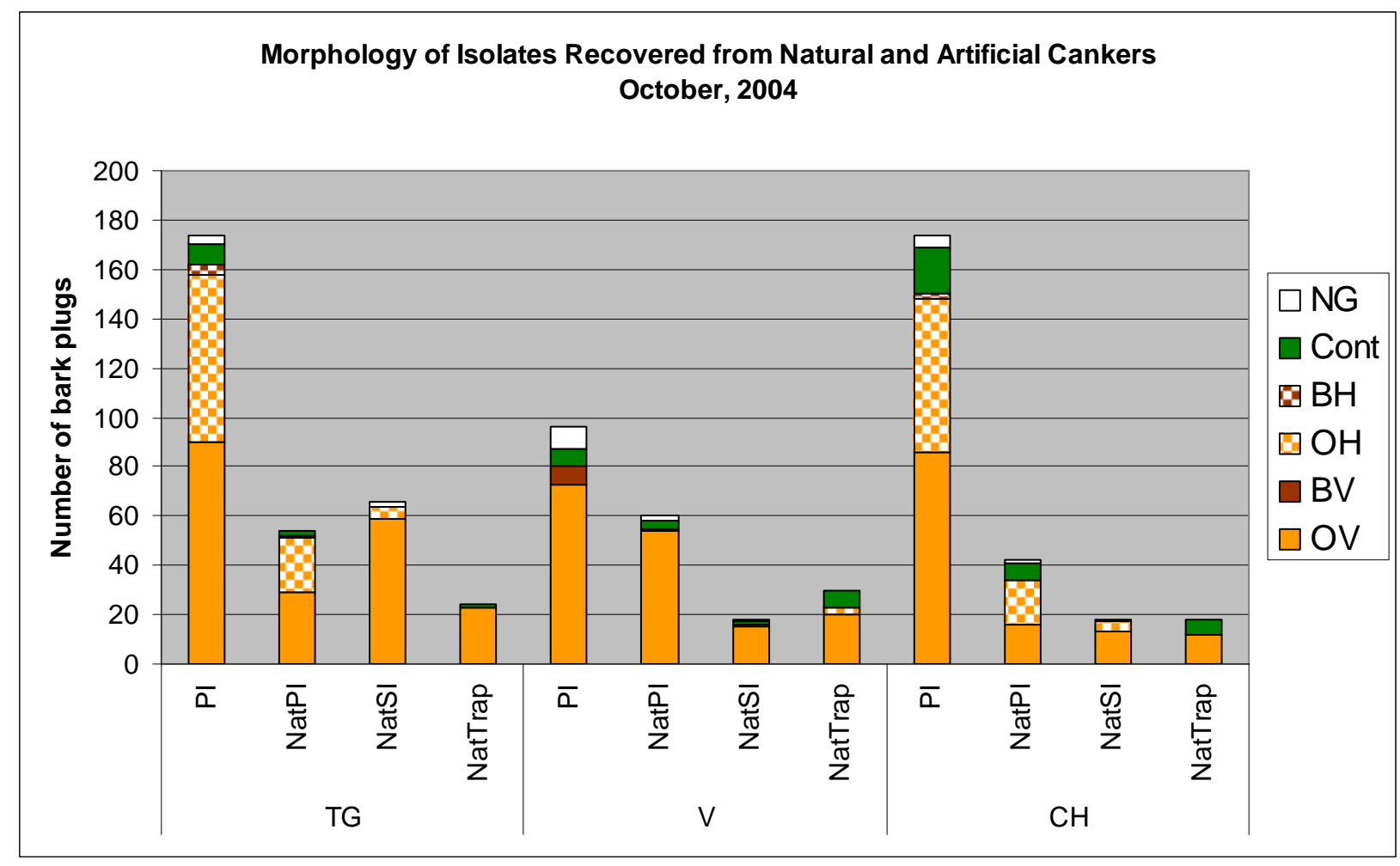

Figure 17. Number of colonies by morphology collected from bark plugs on four types of cankers. PI=punchinitiated; SI=scratch-initiated; NatX=natural canker on X tree. Morphologies: OV=orange-virulent; BV=brownvirulent; $\mathrm{OH}=$ orange-hypovirulent; $\mathrm{BH}=$ brown-hypovirulent; Cont=contaminant; $\mathrm{NG}=$ no growth from bark plug.

The recovery of $\mathrm{HV}$ isolates from two cankers in a $\mathrm{V}$ plot prompted further sampling of those two cankers and two nearby SI cankers in the same V plot in February, 2005. When re- 
sampled, canker D-15-1 yielded $12 \mathrm{OH}$ isolates from 12 plugs, and canker D-16-1 yielded no

HV isolates (Table 7). The two SI cankers on tree D-16 also yielded HV isolates (Table 7). All $\mathrm{HV}$ isolates recovered from these cankers were hygromycin sensitive (hyg ${ }^{\mathrm{S}}$ ).

Table 7. HV recovery from cankers in V plots. $\mathrm{n}=$ number of bark plugs collected; Other=non-C. parasitica isolates. See Fig. 17 for other abbreviations.

\begin{tabular}{|c|c|c|c|c|c|c|c|c|c|c|c|c|}
\hline \multirow[b]{2}{*}{ Canker } & \multicolumn{6}{|c|}{ October, 2004} & \multicolumn{6}{|c|}{ February, 2005} \\
\hline & $n$ & OV & BV & $\mathrm{OH}$ & $\mathrm{BH}$ & Other & $\mathrm{n}$ & OV & BV & $\mathrm{OH}$ & $\mathrm{BH}$ & Other \\
\hline D-15-1 & 6 & 1 & 0 & 3 & 0 & 2 & 12 & 0 & 0 & 12 & 0 & 0 \\
\hline D-16-1 & 6 & 5 & 0 & 1 & 0 & 0 & 12 & 8 & 0 & 0 & 0 & 4 \\
\hline D-16-SI(50cm) & 0 & - & - & - & - & - & 6 & 0 & 0 & 0 & 5 & 1 \\
\hline D-16-SI(150cm) & 0 & - & - & - & - & - & 6 & 4 & 0 & 2 & 0 & 0 \\
\hline
\end{tabular}

Of the 95 orange-hypovirulent $(\mathrm{OH})$ isolates recovered from TG plots, only four (4.2\%) were hygromycin resistant $\left(\mathrm{hyg}^{\mathrm{R}}\right)$, and all five (100\%) of the brown-hypovirulent (BH) isolates were hyg ${ }^{\mathrm{R}}$ (Table 8). All nine hyg ${ }^{\mathrm{R}}$ isolates were recovered from cankers that were treated with TG spermatia. No hyg ${ }^{\mathrm{R}}$ hypovirulent isolates were recovered from $\mathrm{CH}$ or V plots (Table 8).

None of 83 virulent isolates tested from the three plot treatments were hyg $^{\mathrm{R}}$ (Table 8).

Table 8. Morphology and hyg $^{\mathrm{R}}$ of canker isolates. See Fig. 16 for morphology abbreviations.

\begin{tabular}{|c|c|c|c|c|c|}
\hline $\begin{array}{c}\text { Plot } \\
\text { Treatment }\end{array}$ & $\begin{array}{c}\text { Isolate } \\
\text { Morphology }\end{array}$ & $\begin{array}{c}\mathbf{n} \\
\text { Isolates }\end{array}$ & $\begin{array}{l}\text { \# Tested } \\
\text { for Hyg }\end{array}$ & \# Hyg $^{\mathrm{R}}$ & $\% \mathrm{Hyg}^{\mathrm{R}}$ \\
\hline \multirow{4}{*}{ TG } & OV & 201 & 34 & 0 & 0 \\
\hline & BV & 0 & - & - & - \\
\hline & $\mathrm{OH}$ & 95 & 95 & 4 & 4.2 \\
\hline & $\mathrm{BH}$ & 5 & 5 & 5 & 100 \\
\hline \multirow{4}{*}{ V } & OV & 156 & 18 & 0 & 0 \\
\hline & BV & 8 & 3 & 0 & 0 \\
\hline & $\mathrm{OH}$ & 4 & 4 & 0 & 0 \\
\hline & $\mathrm{BH}$ & 0 & - & - & - \\
\hline \multirow{4}{*}{$\mathrm{CH}$} & OV & 127 & 28 & 0 & 0 \\
\hline & BV & 0 & - & - & - \\
\hline & $\mathrm{OH}$ & 84 & 84 & 0 & 0 \\
\hline & $\mathrm{BH}$ & 2 & 2 & 0 & 0 \\
\hline
\end{tabular}


Cankers that were punch-initiated to establish infections using wild-type isolates PC17 and PC23 yielded higher HV recovery rates than those established with other wild-type isolates (Fig. 18A). These field results were similar to the high rates of hypovirus acquisition also seen under laboratory conditions (Fig. 18B). Hypoviruses also were transmitted to field cankers initiated with strains that were unable to receive hypovirus via anastomosis under laboratory conditions. For example, PC27 did not acquire hypovirus from either introduced strain in the laboratory after 5 replications per pairing (Fig. 18B), but an average of $\sim 40 \%$ of isolates recovered from field cankers initiated with PC27 were hypovirulent (Fig. 18A).

\section{Ascospore Sampling}

Bark discs were collected from a total of 87 cankers from all three plot treatments. Of these cankers, no mature perithecia were found on discs from 19 of the cankers. From the remaining 68 cankers (45=TG, 10=V, 13=CH), a total of 9821 ascospores were cultured and the resulting isolates were scored for morphology. Numerous unexpected variations in morphology were observed and will be presented later.

Outcrossing to MAT-1 treatment strains could be assessed by the presence of brown ascospore colonies, while outcrossing to non-transgenic EP155-based strains could not be morphologically distinguished from outcrossing to indigenous C. parasitica strains. In TG plots, however, the presence of hyg ${ }^{\mathrm{R}}$ individuals in perithecia that yielded only orange isolates indicates spermatization by EP155/pxHE7. Table 9 contains outcrossing data from the three plot treatments on a canker and perithecia basis. 

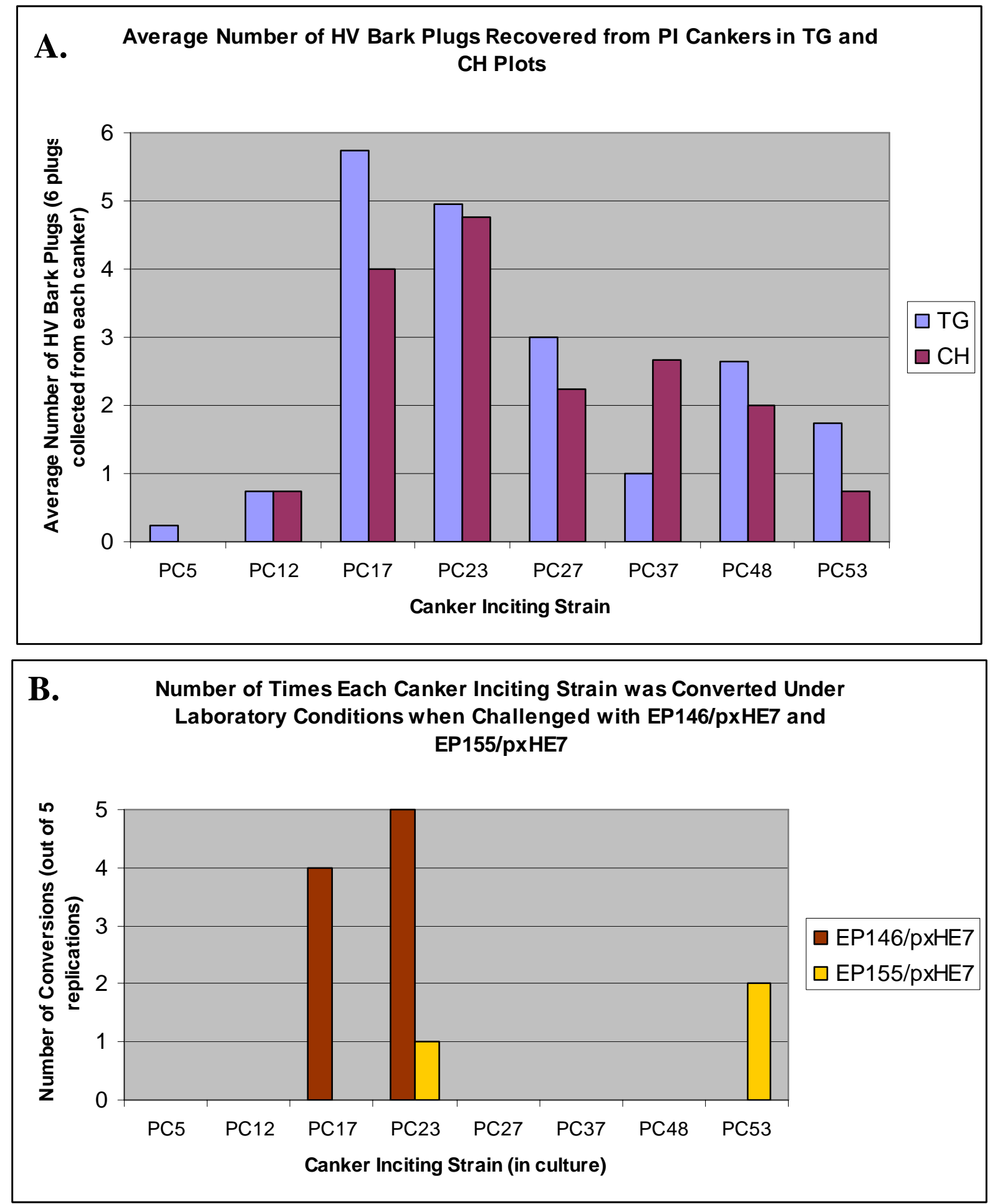

Figure 18. A. Average number of HV bark plugs recovered from cankers initiated with PC isolates collected from the site. Six bark plugs were removed from each of 2-4 cankers, so the highest possible value for one canker would be 6. B. Number of conversions out of 5 laboratory pairings between canker inciting strains and either EP146/pxHE7 or EP155/pxHE7. 
When considering all three plot treatments, EP146-based isolates spermatized 93.3\%, $50 \%$, and $75 \%$ of PI cankers in TG, $\mathrm{V}$, and $\mathrm{CH}$ plots, respectively. Brown ascospore colonies were isolated at lower percentages from natural cankers (approximately 16.7\%, 11.1\%, and 20\% of treated natural cankers in TG, V, and $\mathrm{CH}$ plots, respectively). Hygromycin-resistant ascospore progeny were isolated from $71.4 \%$ of PI cankers that were determined to outcross to EP155/pxHE7 (Table 9). Approximately 30\% of natural cankers also yielded hygromycin resistant individuals, even when they did not outcross to the MAT-1 transgenic strain (Table 9).

Although painted cankers (both PI cankers and natural cankers on PI trees) comprised the majority of outcrossed cankers, two natural cankers on SI trees also showed evidence of outcrossing. One of these cankers occurred in a TG plot and yielded OV, BV, OH, and BH ascospores. The other untreated natural canker that showed evidence of outcrossing occurred in a V plot and yielded OV and BV ascospores, signifying spermatization by the EP146 brownpigmented strain employed in $\mathrm{V}$ plots. There was no evidence of outcrossing in natural cankers that occurred on trap trees.

Table 9. Outcrossing percentages for three main treatments by canker and perithecia levels.

\begin{tabular}{|c|c|c|c|c|c|c|c|c|c|c|}
\hline \multirow[b]{2}{*}{$\begin{array}{c}\text { Canker } \\
\text { (Spermatia) }\end{array}$} & \multirow[b]{2}{*}{$\begin{array}{c}\text { Sampling } \\
\text { Unit }\end{array}$} & \multicolumn{3}{|c|}{ TG } & \multicolumn{3}{|c|}{ v } & \multicolumn{3}{|c|}{$\mathrm{CH}$} \\
\hline & & $\mathbf{n}$ & $\begin{array}{c}\% \\
\text { with } \\
\text { brown }\end{array}$ & $\begin{array}{c}\% \\
\text { with } \\
\text { hyg }^{\mathrm{R}}\end{array}$ & $\mathbf{n}$ & $\begin{array}{l}\% \text { with } \\
\text { brown }\end{array}$ & $\begin{array}{c}\% \\
\text { with } \\
\text { hyg }^{\mathrm{R}}\end{array}$ & $\mathrm{n}$ & $\begin{array}{l}\% \text { with } \\
\text { brown }\end{array}$ & $\begin{array}{c}\% \\
\text { with } \\
\text { hyg }^{\mathrm{R}}\end{array}$ \\
\hline \multirow{2}{*}{$\begin{array}{c}\text { PI Cankers } \\
\text { (EP146-based } \\
\text { strains) }\end{array}$} & Canker & 15 & 93.3 & 93.3 & 4 & 50 & 0 & 4 & 75 & 0 \\
\hline & Perithecia & 105 & 68.6 & 68.6 & 14 & 57.1 & 0 & 20 & 55 & 0 \\
\hline \multirow{2}{*}{$\begin{array}{c}\text { PI Cankers } \\
\text { (EP155-based } \\
\text { strains) }\end{array}$} & Canker & 14 & 14.3 & 71.4 & 4 & - & - & 4 & 0 & 0 \\
\hline & Perithecia & 95 & 2.1 & 25.3 & 0 & - & - & 5 & 0 & 0 \\
\hline \multirow{2}{*}{$\begin{array}{l}\text { Natural Cankers } \\
\text { (Undetermined) }\end{array}$} & Canker & 24 & 16.7 & 29.2 & 9 & 11.1 & 0 & 10 & 20 & 0 \\
\hline & Perithecia & 88 & 13.6 & 21.6 & 34 & 14.7 & 0 & 32 & 31.3 & 0 \\
\hline
\end{tabular}

Morphology ratios of ascospore colonies from successful EP146-based spermatizations in all three plot treatments were summed and compared to expected Mendelian ratios using a chi- 
squared goodness-of-fit test. Outcrossing to EP146/pxHE7 was expected to yield a ratio of 1:1:1:1 (OV:BV:OH:BH), while EP146/tpTE7 and EP146 were expected to yield a ratio of 1:1 (OV:BV). Observed ascospore isolate ratios from EP146/pxHE7 crosses were significantly different from an expected Mendelian ratio $\left(\mathrm{P}>\chi^{2}=<0.001\right)$, while the ratio of OV to BV ascospores in $\mathrm{V}\left(\mathrm{P}>\chi^{2}=0.459\right)$ and $\mathrm{CH}\left(\mathrm{P}>\chi^{2}=0.999\right)$ plots were not shown to differ significantly from expected values (Fig 19).

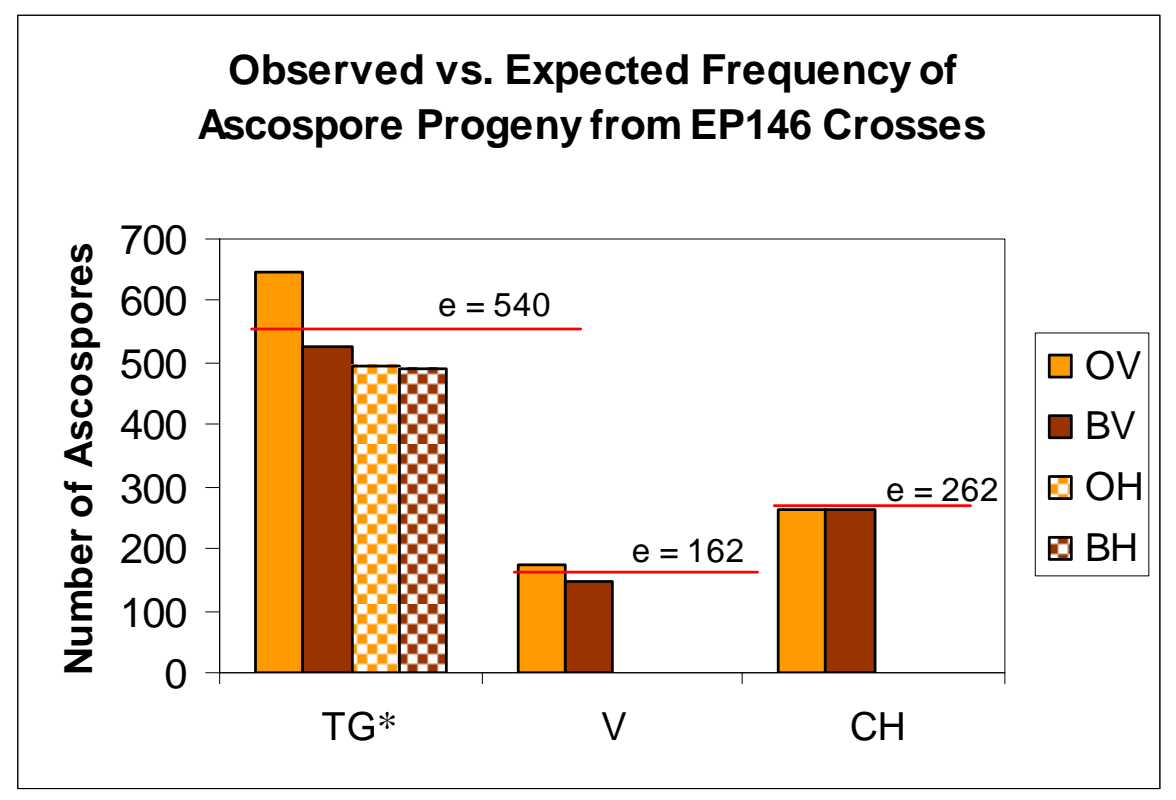

Figure 19. Observed (bars) and expected (red lines) values for ascospore progeny from EP146 crosses in each of the main treatments. e=expected value; See Fig. 17 for other abbreviations. “*” indicates a distribution significantly different from an expected Mendelian ratio.

Hypovirulent ascospores (HVAs) were only isolated from TG plots (Table 10). All OH and $\mathrm{BH}$ ascospore colonies that were tested were hyg $^{\mathrm{R}}$ (Table 10) and contained dsRNA, confirming hypovirulence (Fig. 26, lanes 6, 7, and 17). No morphologically-definitive HVAs were isolated from cankers spermatized by EP155/pxHE7, though 20\% of the ascospore isolates from these crosses were hyg ${ }^{\mathrm{R}}$. In laboratory crosses, canker initiating wild-type isolate PC27 was determined to be MAT-1 (i.e. to be spermatized by EP155), but two perithecia from 
cankers initiated with this isolate outcrossed to EP146/pxHE7 and produced ascospore colonies that were hypovirulent and brown (Table 10). Amplification of MAT specific regions by PCR showed the presence of both MAT-1 and MAT-2 specific sequences in isolate PC27 (data not shown).

Although no morphologically-definitive HVAs were isolated from a canker that was thought to outcross to EP155/pxHE7, 19.9\% of OV ascospore isolates tested from such crosses were hyg $^{\mathrm{R}}$ (Table 10). All 25 ascospore colonies from each of 13 selected perithecia were tested to assess the ratio at which hyg ${ }^{\mathrm{R}}$ individuals occurred in EP155/pxHE7 offspring. Seven of the thirteen perithecia contained no hyg ${ }^{\mathrm{R}}$ individuals. The average ratio of hyg $^{\mathrm{R}}$ :hyg ${ }^{\mathrm{S}}$ in the remaining six perithecia was approximately 1:1.6 (Table 11). Although this does not conform to a 1:1 ratio $\left(\mathrm{P}>\chi^{2}=0.006\right)$, some of the perithecia exhibited a ratio very close to 1:1 (Table 11$)$. No ascospore progeny from an EP155/pxHE7 cross had distinct bands of dsRNA, though some of the variant isolates tested (\#19Var and \#20Var, Fig. 25B) did show faint bands compared to morphologically distinct hypovirulent isolates (Fig. 26, lanes 12 and 13).

The pathogenicity of unexpected hyg ${ }^{\mathrm{R}} \mathrm{OV}$ isolates was tested by inoculating them into apples and measuring the subsequent necrotic lesions ( $\mathrm{n}=5$ lesions). Although an $\mathrm{OH}$ ascospore isolate and one hyg ${ }^{\mathrm{R}} \mathrm{OV}$ isolate showed significantly smaller lesions after seven days $(\mathrm{P}>\mathrm{F}=<.0001$; Appendix $\mathrm{C})$, lesion diameters of $\mathrm{hyg}^{\mathrm{R}} \mathrm{OV}$ isolates and $\mathrm{HV}$ isolates were not significantly different from most virus-free or virus-infected controls after 14 and 21 days. However, one hyg ${ }^{\mathrm{S}} \mathrm{OV}$ isolate did show significantly less growth compared to other isolates and controls after each incubation period $(\mathrm{P}>\mathrm{F}=<.0001, \mathrm{P}>\mathrm{F}=<.0001, \mathrm{P}>\mathrm{F}=.0479$ for 7, 14, and 21 days respectively). This hyg ${ }^{\mathrm{S}}$ isolate was dsRNA free (Fig. 26, lane 3). 
Table 10. Number of ascospore colonies isolated from PI and natural cankers. Cankers are listed according to compatible (opposite) mating type spermatia. Colonies are separated by whether they outcrossed to the treatment inoculum or natural inoculum. "Other" category included non-C. parasitica isolates and no germination. See Fig. 15 for abbreviations.

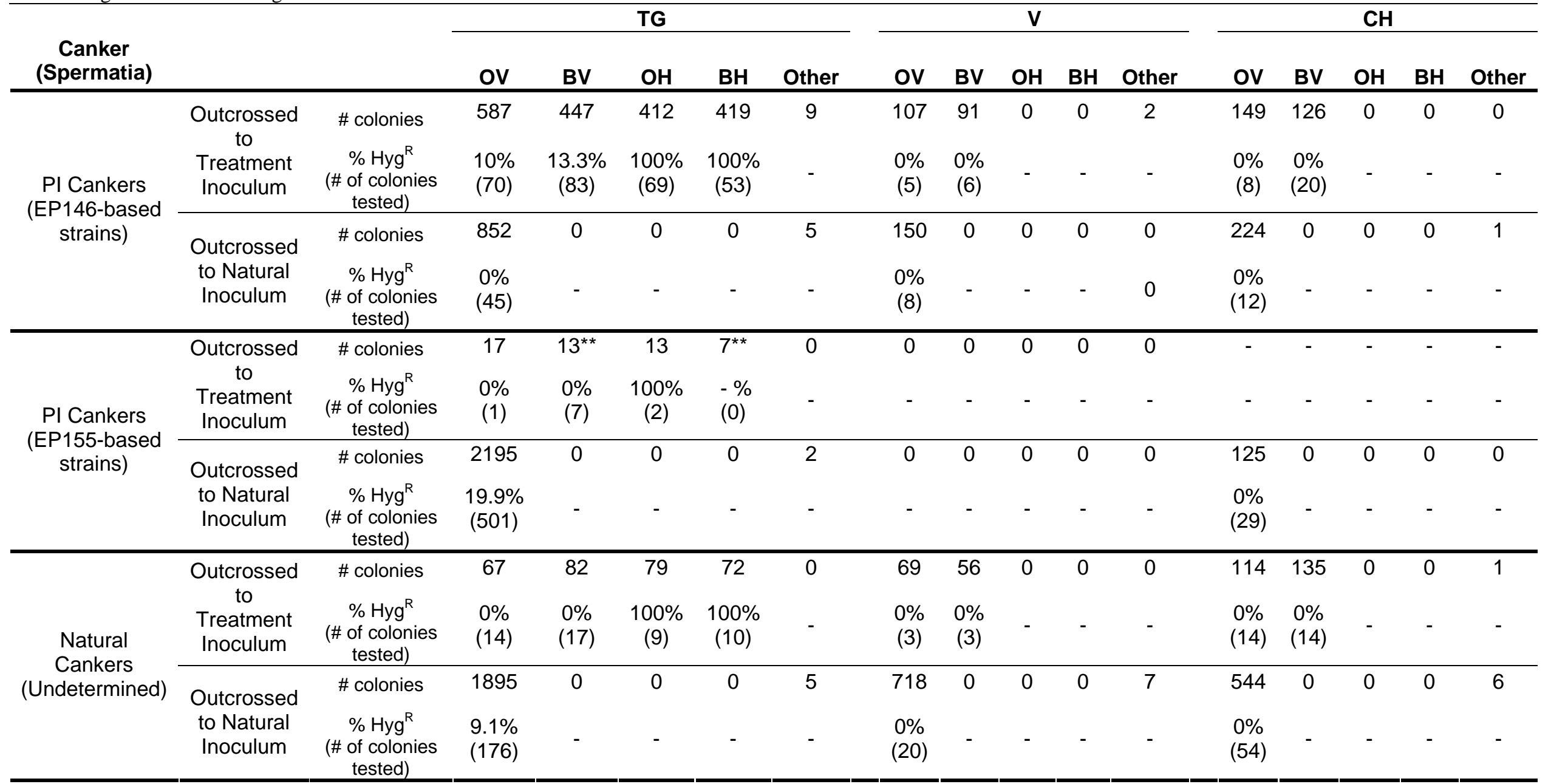

* - Since no morphologically distinct hypovirulent colonies were collected from EP155/pxHE7 crosses, colonies could not be distinguished by whether they outcrossed to the treatment inoculum or not. There also was no marker to determine whether colonies from the $\mathrm{V}$ or $\mathrm{CH}$ plots outcrossed to the treatment inoculum or natural inoculum.

** - Indicates BV and BH colonies isolated from a suspected EP155/pxHE7 cross. These colonies were isolated from a canker initiated with isolate PC27 which contained both MAT alleles when tested in the laboratory. 
Table 11. Ratios of hyg ${ }^{\mathrm{R}}$ hyg $^{\mathrm{S}}$ ascospores from EP155/pxHE7 crosses. PI=Punch-initiated cankers at a height of either $50 \mathrm{~cm}$ or $150 \mathrm{~cm}$ on the tree.

\begin{tabular}{|c|c|c|c|c|c|c|}
\hline Plot & Tree & Canker & Perithecia & \# hyg $^{\mathrm{R}}$ & \#hyg $^{\text {s }}$ & $\begin{array}{c}\text { Ratio } \\
\left.\text { (hyg }^{R}: h^{S} g^{S}\right)\end{array}$ \\
\hline$L$ & 3 & $\mathrm{PI}-150 \mathrm{~cm}$ & 2 & 13 & 12 & $1: 0.92$ \\
\hline L & 3 & $\mathrm{PI}-150 \mathrm{~cm}$ & 5 & 6 & 19 & $1: 3.2$ \\
\hline L & 3 & $\mathrm{PI}-150 \mathrm{~cm}$ & 6 & 10 & 15 & $1: 1.5$ \\
\hline L & 3 & $\mathrm{PI}-150 \mathrm{~cm}$ & 8 & 6 & 19 & $1: 3.2$ \\
\hline L & 3 & $\mathrm{PI}-150 \mathrm{~cm}$ & 10 & 12 & 13 & 1:1.1 \\
\hline \multirow[t]{2}{*}{ A } & 12 & $\mathrm{PI}-50 \mathrm{~cm}$ & 3 & 11 & 14 & $1: 1.3$ \\
\hline & & & $\begin{array}{l}\text { Total } \\
\text { an ratic }\end{array}$ & 58 & 92 & $1: 1.6^{*}$ \\
\hline
\end{tabular}

\section{Transmission of dsRNA to other v-c types}

Inheritance of the cDNA transgene by isolates of different v-c types was assessed by comparing the hypovirus transmission of parental HV strains to that of selected HVAs from cankers of multiple genetic backgrounds (PI cankers and natural cankers). When paired in culture, parental strains EP146/pxHE7 and EP155/pxHE7 could not transmit their hypovirus via anastomosis to 17 of the $29 \mathrm{v}$-c types delineated at the beginning of the study (Table 4).

Therefore, the 17 previously non-converted v-c types were paired five times in the laboratory with 18 selected HVA isolates to evaluate the hypovirus transmission capability of the HV sexual progeny. Hypovirulent ascospores consistently transmitted (4-5 transmissions out of 5 pairings) their hypovirus to 12 of the $17 \mathrm{v}$-c types while three additional v-c types became infected at least once (Table 12;Fig. 20). Two v-c types were unable to be converted by any of the 18 HVA isolates (Table 12;Fig. 20). 

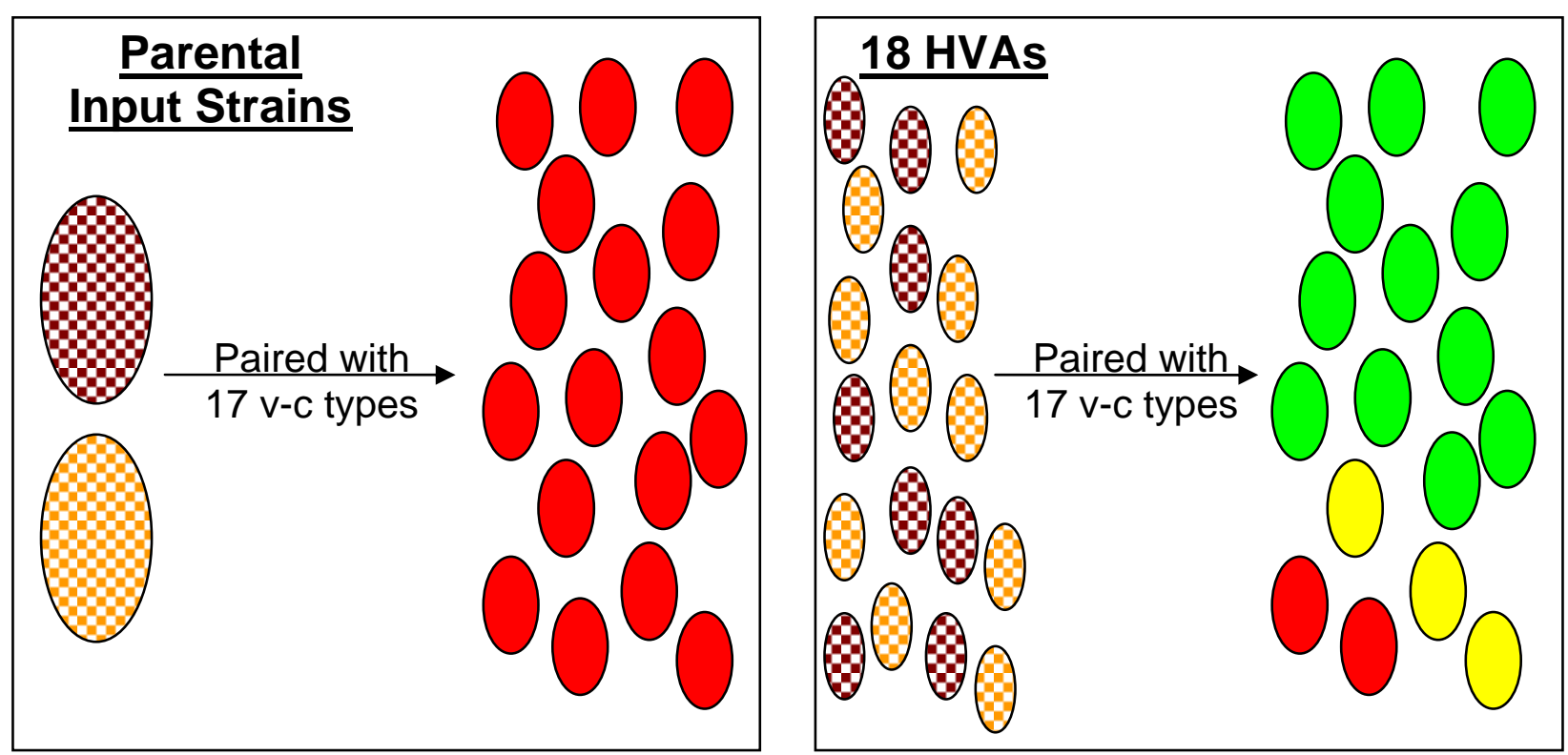

Figure 20. Dissemination of cDNA to different v-c groups after sexual recombination. Ovals in the left side of each box represent parent strains EP146/pxHE7 and EP155/pxHE7 (left box) or HVA isolates from TG plots (right box). The 17 ovals in the right of each box represent 17 separate v-c types isolated from the site. The color of the 17 ovals represents the number of times that isolate was able to acquire hypovirus via anastomosis from either the parental strains (left box) or collectively from HVA isolates (right box). Green=4-5 conversions; Yellow=1-3 conversions; Red $=0$ conversions.

\section{Hypovirulent Conidia Production}

Bark discs were collected from scratch-initiated cankers in each main treatment set to sample pycnidia and analyze morphology of single conidial isolates. Conidia were isolated from five TG cankers and four V cankers. No viable pycnidia were found on bark discs sampled from CH cankers. All pycnidia sampled from TG plots yielded approximately $100 \%$ HV conidia with the same pigmentation as the hypovirulent strain used to initiate the infection (Fig. 21).

Although three of the four $\mathrm{V}$ cankers analyzed yielded all $\mathrm{V}$ isolates of the same pigmentation as the treatment strain, one canker yielded approximately $80 \%$ BH conidia and $4 \% \mathrm{OH}$ conidia

(Fig. 21). All HV conidial isolates tested from TG plots were $\mathrm{hyg}^{\mathrm{R}}$, while all $\mathrm{HV}$ isolates tested from the V canker were hyg $\mathrm{S}$. 
Table 12. Number of hypovirus transmissions out of 5 replications per pairing. PC isolates across the top were collected from the site and were unable to acquire hypovirus via anastomosis from introduced HV strains (EP146/pxHE7 and EP155/pxHE7). Hypovirulent ascospores were collected from cankers initiated by one of the female parents. They are listed in the order they were tested; when an HVA isolated converted a PC isolate 4 or 5 times, that isolate was dropped from further analysis. Female parent strains represent the most common v-c types isolated from the site and were used to initiate PI cankers. Natural cankers of unknown v-c type also were used as female parents.

\begin{tabular}{|c|c|c|c|c|c|c|c|c|c|c|c|c|c|c|c|c|c|c|}
\hline $\begin{array}{c}\text { HV } \\
\text { Ascospore }\end{array}$ & $\begin{array}{c}\text { Female } \\
\text { Parent }\end{array}$ & PC5 & PC6 & PC12 & PC18 & PC19 & PC21 & PC27 & PC29 & PC37 & PC39 & PC45 & PC47 & PC50 & PC51 & PC52 & PC54 & PC58 \\
\hline HVA - 62 & PC17 & 0 & 2 & 0 & 0 & 0 & 0 & 0 & 0 & 0 & 0 & 0 & 0 & 0 & 0 & 0 & 0 & 0 \\
\hline HVA - 37 & PC37 & 0 & 3 & 1 & 5 & 1 & 0 & 0 & 3 & 4 & 0 & 0 & 0 & 0 & 0 & 0 & 0 & 0 \\
\hline HVA - 87 & Natural & 0 & 2 & 3 & 0 & 0 & 0 & 0 & 5 & 0 & 0 & 0 & 0 & 0 & 0 & 0 & 0 & 4 \\
\hline HVA - 45 & PC17 & 2 & 1 & 1 & 0 & 1 & 0 & 0 & 0 & 0 & 0 & 0 & 0 & 0 & 0 & 0 & 0 & 2 \\
\hline HVA - 6 & PC12 & 2 & 4 & 5 & 0 & 0 & 0 & 3 & 0 & 0 & 0 & 1 & 0 & 0 & 0 & 0 & 0 & 0 \\
\hline HVA - 12 & PC48 & 0 & 5 & 1 & - & 0 & 5 & 1 & - & - & 0 & 5 & 0 & 0 & 0 & 0 & 0 & - \\
\hline HVA - 14 & PC27 & 4 & - & - & - & 0 & 0 & 3 & - & - & 0 & 0 & 0 & 0 & 0 & 0 & 0 & - \\
\hline HVA - 38 & PC37 & 0 & - & - & - & 0 & 0 & 0 & - & - & 0 & 0 & 1 & 0 & 0 & 0 & 0 & - \\
\hline HVA - 41 & PC17 & 1 & - & - & - & 1 & 0 & 0 & - & - & 0 & 0 & 0 & 0 & 0 & 0 & 1 & - \\
\hline HVA - 5 & PC12 & 5 & - & - & - & 0 & - & 5 & - & - & 3 & 5 & 4 & 0 & 0 & 0 & 0 & - \\
\hline HVA - 8 & Natural & 0 & - & - & - & 0 & - & 0 & - & - & 0 & 0 & 2 & 0 & 0 & 0 & 0 & - \\
\hline HVA - 11 & PC27 & - & - & - & - & 0 & - & - & - & - & 4 & - & 4 & 0 & 0 & 0 & 0 & - \\
\hline HVA - 17 & PC48 & - & - & - & - & 1 & - & - & - & - & 0 & - & - & 0 & 0 & 0 & 1 & - \\
\hline HVA - 31 & PC37 & - & - & - & - & 0 & - & - & - & - & 0 & - & - & 0 & 0 & 3 & 2 & - \\
\hline HVA - 60 & PC17 & - & - & - & - & 0 & - & - & - & - & 0 & - & - & 0 & 0 & 0 & 0 & - \\
\hline HVA - 10 & PC27 & - & - & - & - & 0 & - & - & - & - & - & - & - & 0 & 0 & 0 & 0 & - \\
\hline HVA - 3 & Natural & - & - & - & - & 0 & - & - & - & - & - & - & - & 0 & 0 & 0 & 0 & - \\
\hline HVA - 13 & PC48 & - & - & - & - & 1 & - & - & - & - & - & - & - & 0 & 0 & 0 & 0 & - \\
\hline
\end{tabular}




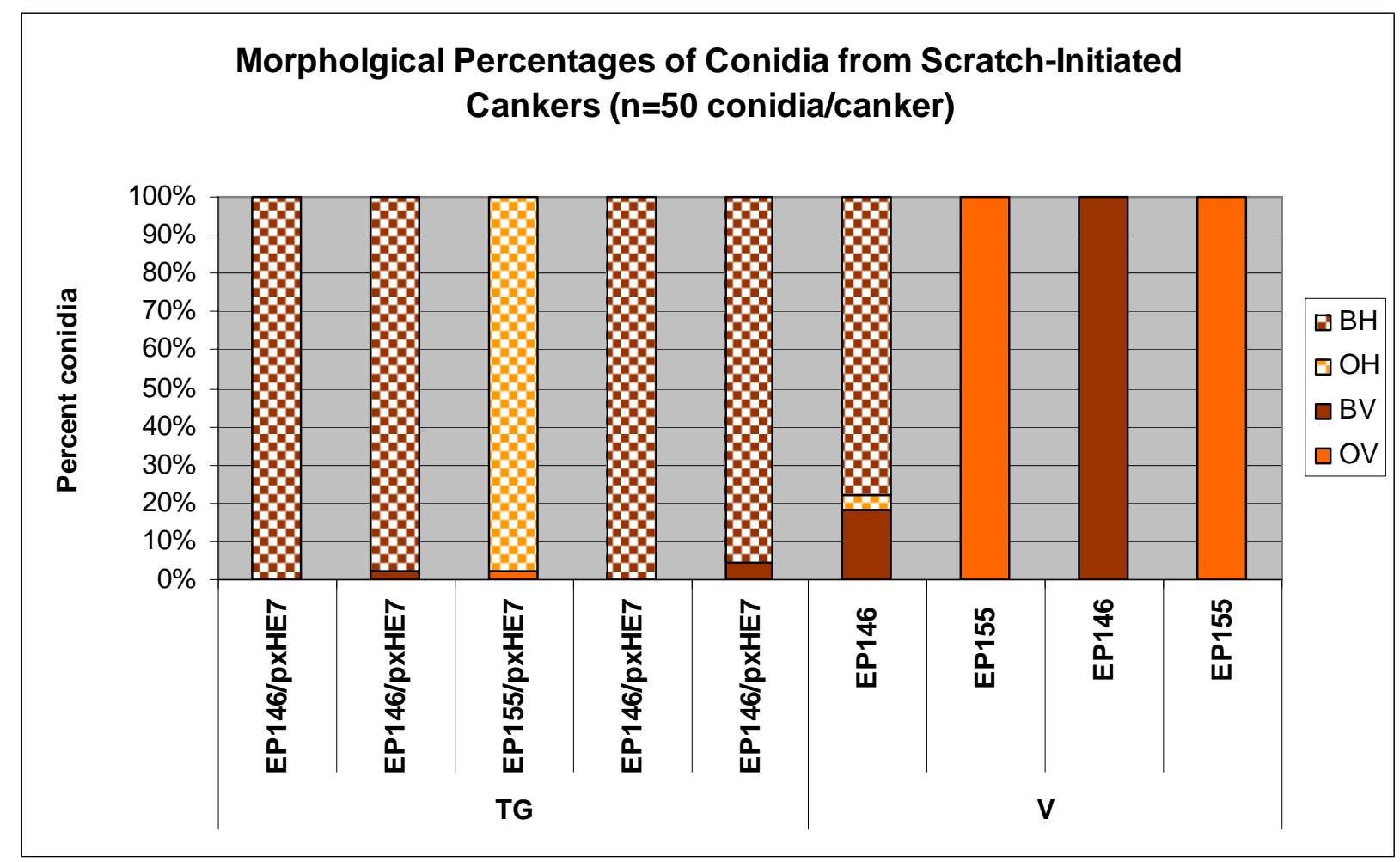

Figure 21. Percentages of conidial isolates by morphology collected from scratch-initiated cankers. The first 5 columns represent cankers from TG plots labeled by canker initiating strains. The last 4 columns represent scratchinitiated cankers from V plots. No viable pycnidia were isolated from scratch-initiated cankers in CH plots. See Fig. 16 for morphology abbreviations.

Single germinating conidia were isolated from laboratory cultures of treatment strains EP146/pxHE7 and EP155/pxHE7 several times during the experiment to examine the mitotic stability of the cDNA transgene. Strain EP146/pxHE7 had a 100\% inheritance rate for two of the three separate single spore isolations, though on the third test in July, 2004 only 13 of the 30 spores collected were the expected BH phenotype. Strain EP155/pxHE7 had an approximate 100\% inheritance rate four out of the five isolations. The final isolation in February, 2005 resulted in $35 \%$ of isolates with a pink pigment and $5 \%$ with a variant $\mathrm{HV}$ (VarH) morphology (Fig. 22). These variant isolates contained dsRNA fragments of similar size to EP155/pxHE7 (Fig. 26, lanes 4, 5, 8, and 9). The pathogenicity of pink and VarH isolates was assessed by 
comparing their growth in apples to the growth of morphologically distinct $\mathrm{OH}$ conidia and V/HV parental controls. Pink strains caused significantly smaller lesions after all three incubation periods than did $\mathrm{OH}$ and $\mathrm{OV}$ strains $(\mathrm{P}>\mathrm{F}=<.0001$ for all 3 incubation periods; Fig. 23). Analyses of these data by Scheffe's comparison procedure indicated no significant differences $(\mathrm{P}<0.05)$ in lesion diameter among the pink strains after all three incubation times. Scheffe’s procedure $(\mathrm{P}<0.05)$ also indicated that isolate \#39VarH caused lesions similar in size to those of pink isolates, while isolate \#58VarH was similar to $\mathrm{OH}$ and $\mathrm{OV}$ isolates.

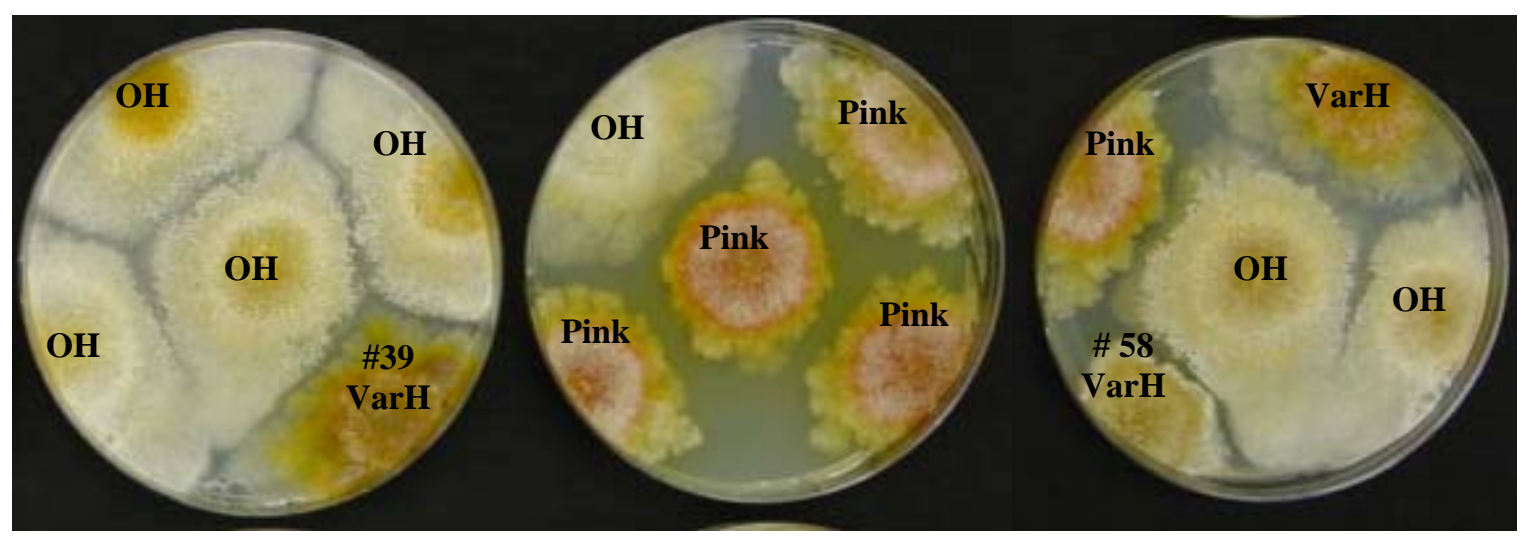

Figure 22. Morphologies of EP155/pxHE7 conidia isolations done in February, 2005. Each isolate is labeled with the assigned morphology. "VarH" and "Pink" refer to morphological variations. See Fig. 15 and for other abbreviations. 


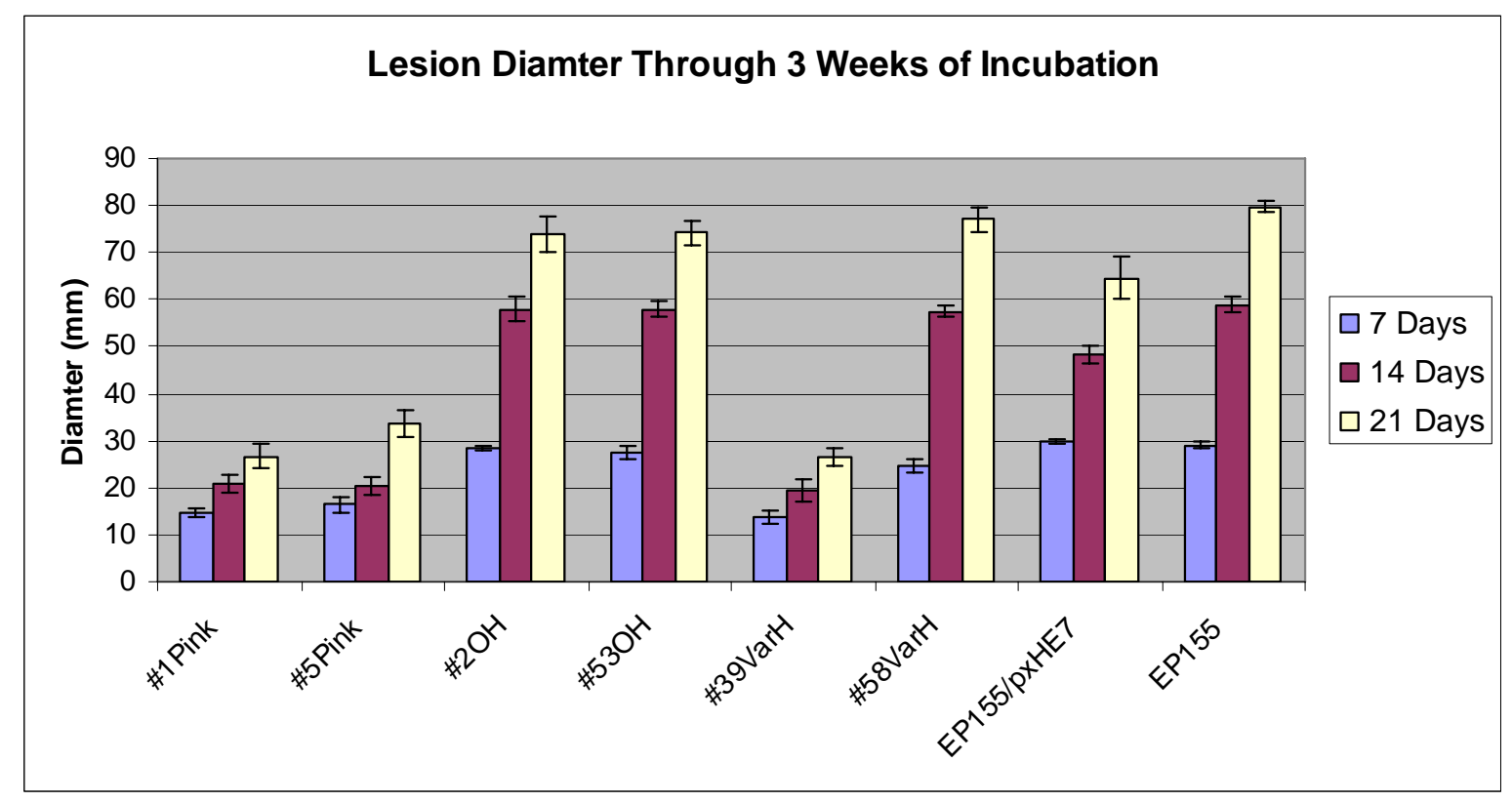

Figure 23. Apple lesion diameters after inoculation with various EP155/pxHE7 conidia isolates. Error bars show $+/-1$ SE . See Fig. 22 for culture morphologies.

\section{Variant Ascospore Isolates}

While most ascospore colonies cultured from the site displayed morphologies typical of the four expected classes, several isolates displayed unusual morphologies and were designated as “variant.” Most variants were scored as virulent (Fig. 24A). One type of variant was scored as hypovirulent due to the restricted growth and pycnidial production observed in culture (Fig 24B). Some morphologically variant isolates scored as virulent (\#9Var and \#11Var, Fig. 25A) contained dsRNA (Fig. 26, lanes 10 and 11) and were hyg ${ }^{\mathrm{R}}$ (Fig. 25). 

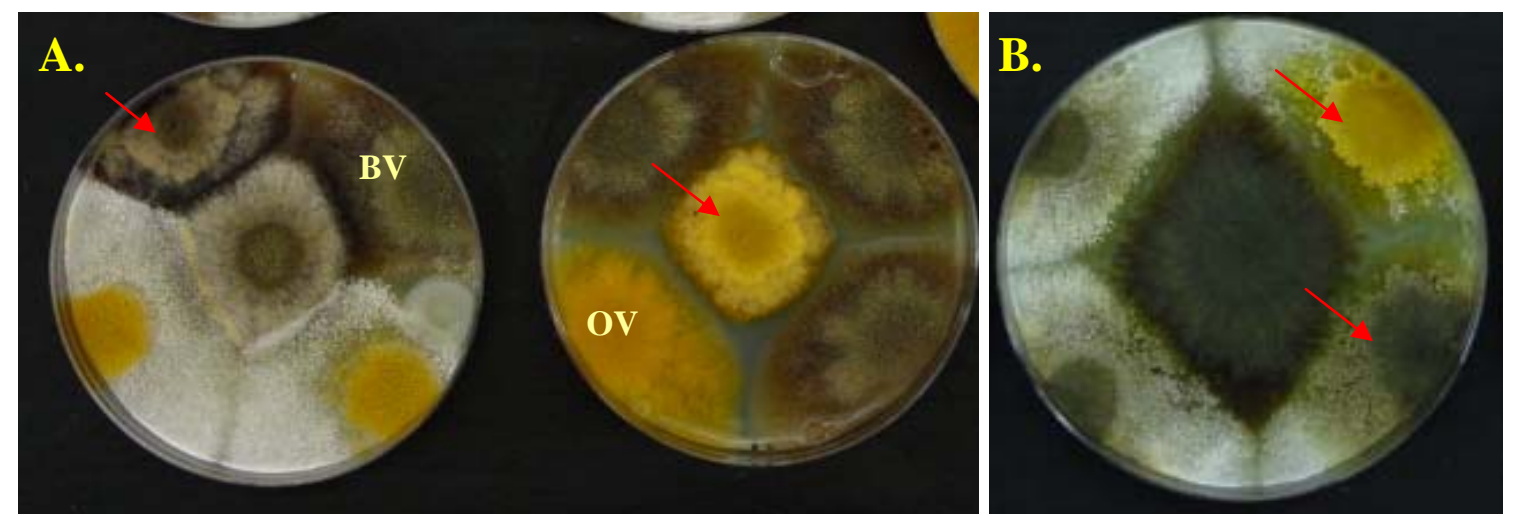

Figure 24. Ascospore colonies showing variation in morphology (red arrows). A. Variant morphologies scored as virulent. Notice the difference between variants and their "normal" OV/BV counterparts. B. Variants scored as hypovirulent. Notice restricted pigmentation and hyphal growth on the periphery of colonies.

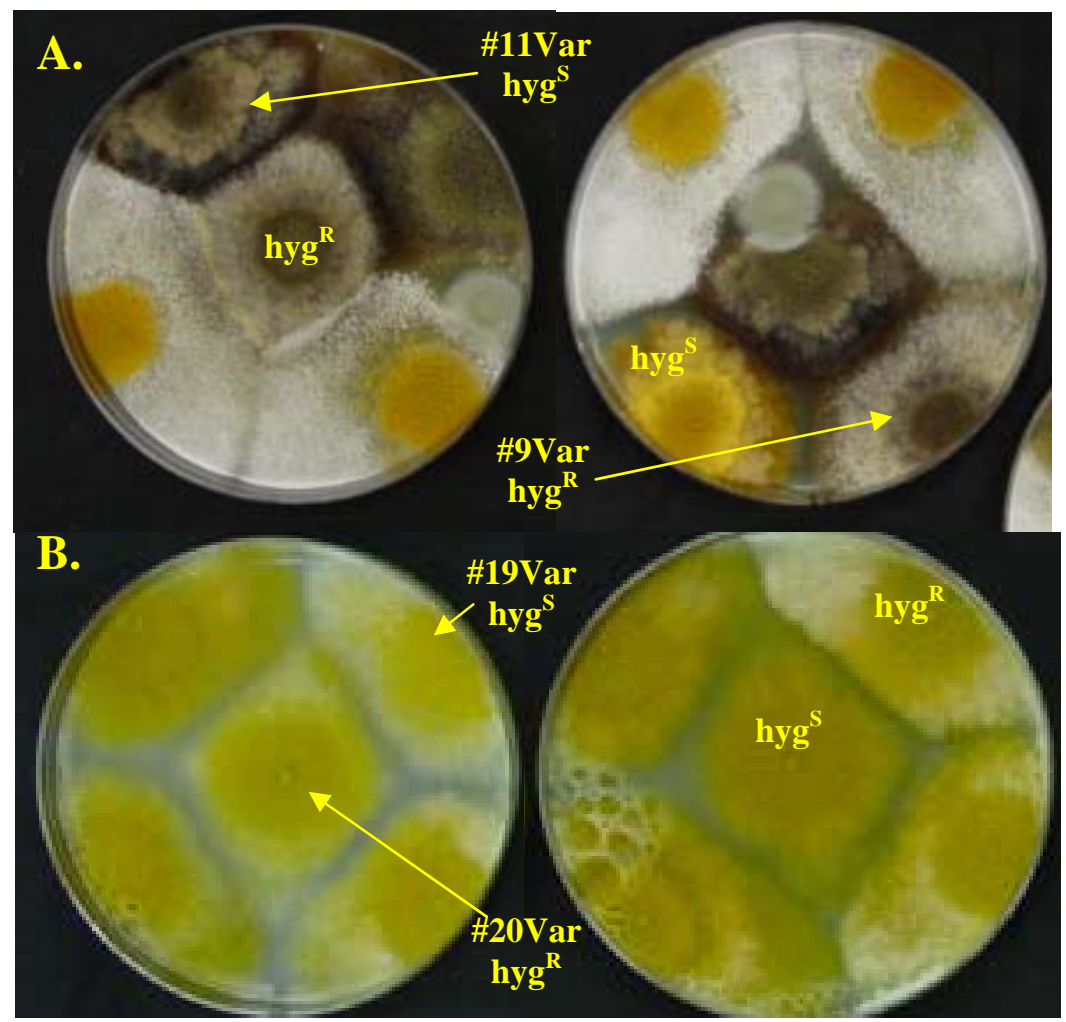

Figure 25. Hyg $^{\mathrm{R}}$ and hyg ${ }^{\mathrm{S}}$ variant isolates. A. Colonies from perithecia that outcrossed to EP146/pxHE7. B. Colonies from perithecia that were thought to outcross to EP155/pxHE7. Those labeled with a number and arrow were tested for dsRNA content. 


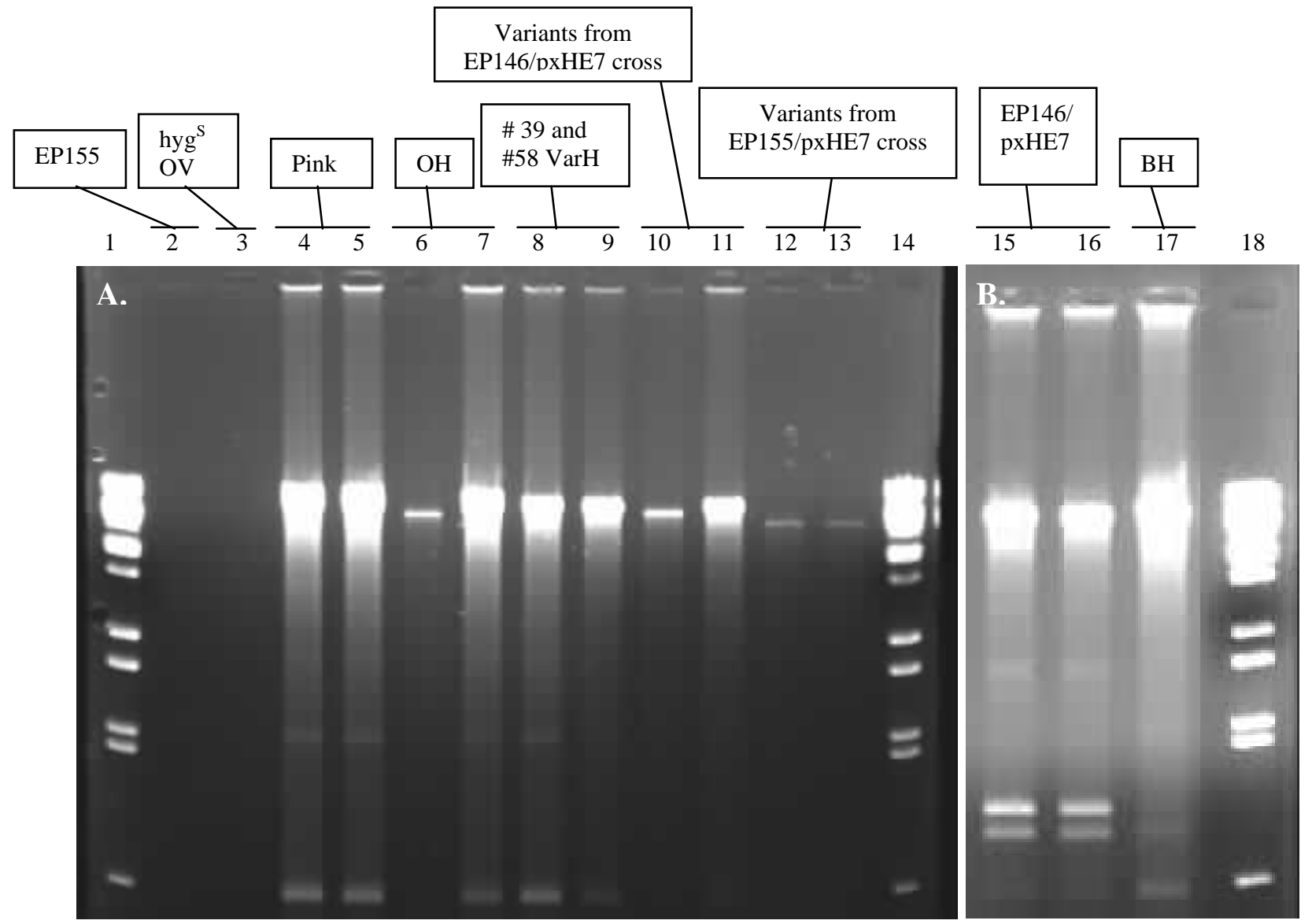

Figure 26. Agarose gel electrophoresis of dsRNA. Lanes 1, 14, 18 contain DNA ladders for relative size

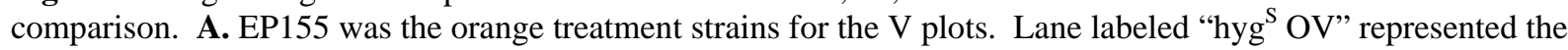
less pathogenic ascospore isolate from the apple pathogenicity test (Fig. 19). Pink, OH, and VarH isolates were from EP155/pxHE7 conidial isolations (Fig. 23). Variants were from EP146/pxHE7 and EP155/pxHE7 crosses (Fig. 26). B. EP146/pxHE7 was the brown treatment strain for the TG plots. BH was a brown-hypovirulent ascospore isolate. 


\section{Discussion}

Transgenic organisms have been used as biological control agents against a myriad of pests. Insecticidal Bacillus thuringiensis (Bt) genes introduced into several agricultural plants have been effective at controlling economically injurious insects (reviewed by Sharma et al., 2003). Introduced Bt genes also have experimentally conferred resistance to loblolly pine (Pinus taeda) and poplar (Populus spp.) species against many defoliating insects (Tang et al., 2003; Yang et al., 2003). A phytotoxin encoding gene introduced into the mildly pathogenic fungus Colletotrichum coccodes experimentally increased its virulence toward agricultural weeds and hence may potentially improve biological weed control (Amsellem et al., 2002). Other than research on transgenic strains of $C$. parasitica, no studies have focused on genetically reducing the virulence of plant pathogenic fungi for the purpose of establishing biological control.

The discovery and characterization of dsRNA hypoviruses was the first step in genetically engineering a hypovirulent strain of $C$. parasitica. A cDNA transgene encoding the viral genome of CHV1-EP713 conferred hypovirulence when introduced into wild-type $C$. parasitica strains (Choi and Nuss, 1992). The stability of this transgene through sexual and asexual reproduction was demonstrated in the laboratory (Chen et al., 1993). These laboratory studies illustrated the increased biological control potential of transgenic strains over their cytoplasmic counterparts because cytoplasmic hypovirulent strains used for biological control transmitted their hypoviruses to conidia at varying rates and did not transmit their hypovirus to ascospore progeny. Two subsequent field release studies demonstrated ascospore inheritance and subsequent dissemination to different v-c groups, as well as the ability of transgenic strains to retard virulent canker expansion (Anagnostakis et al., 1998; Root et al., 2005). However, dissemination of hypovirulent (HV) strains and improvement in stand condition were not 
demonstrated. Strains released in these studies contained a cDNA copy of the CHV1-EP713 hypovirus, which significantly debilitates its fungal host and restricts dissemination of the hypovirulent strain (Chen and Nuss, 1999). The present field experiment focused on the field release of transgenic strains containing a less-debilitating hypovirus CHV1-Euro7. Cytoplasmic $(\mathrm{CH})$ and virulent $(\mathrm{V})$ isogenic strains also were included in the experiment to provide treatment comparisons for the transgenic (TG) strains. The experimental design considered not only hypovirulent ascospore (HVA) production, as with previous transgenic field release studies, but also HV conidia production. Tree condition, hypovirus infection status of cankers, ascospore production, and conidia production also were evaluated after one field season.

Most trees in all plots had healthy crowns after the 2004 season regardless of treatment application. This observation was supported by high tree health rating for most trees; a few canker-bearing trees did exhibit blight symptoms (Table 5). This outcome was anticipated as the artificially initiated cankers were not likely to cause crown symptoms in the time frame encompassed by this study and there were few natural infections that threatened to girdle stems. Scratch-initiated (SI) cankers in V plots expanded significantly more than their TG and $\mathrm{CH}$ counterparts. This is consistent with pathogenicity studies that showed significantly greater growth rates of hypovirus-free virulent strains compared to their isogenic, CHV1-Euro7 infected counterparts (Chen and Nuss, 1999). These large SI cankers will likely girdle the stems more quickly and also will contribute more V inoculum that could contribute to an increase in disease. The significance of generating large stromatic cankers of virulent strains may be evident as the disease develops further.

The system used to rate stroma and callus tissue production indicated that cankers in $\mathrm{CH}$ plots appeared least virulent, followed by cankers in V plots, and then by cankers in TG plots. 
These rankings may not represent actual differences in canker appearance, but subjective differences of the individuals who rated the cankers. Each crew was responsible for rating all cankers in each main treatment, so while readings were consistent within treatments, they may not have been consistent among treatments. Initiated cankers were consistently rated lower than natural cankers, regardless of who rated the cankers. The procedure used to punch-initiate (PI) cankers often caused initial callus production before inoculum could establish a canker, which would have caused lower ratings of some cankers thereby lowering the overall average rating for PI cankers. Ratings of treated cankers were not significantly different from ratings of untreated cankers. Although many cankers treated with conidia (used as spermatia) acquired hypovirus via anastomosis in TG and CH plots, stroma and callus production probably would not have been affected in the short time period of this study. If hypovirus acquisition played a role in canker rating, then there would have been a negative relationship between the number of $\mathrm{HV}$ isolates recovered from a canker and that canker’s rating. Figure 16 shows no such negative relationship. Callus production may become evident on these cankers after future growing seasons when trees grow and develop callus tissue. Also, incorporating measurements of canker growth may identify correlations between HV recovery and canker severity.

Recovery of hygromycin-sensitive (hyg ${ }^{\mathrm{S}}$ ) HV isolates from cankers painted with a slurry containing hygromycin-resistant $\left(\right.$ hyg $\left.^{\mathrm{R}}\right) \mathrm{HV}$ isolates indicates that dsRNA was acquired by canker inciting strains because the hyg $^{\mathrm{R}}$ gene is not transmitted via hyphal anastomosis (Choi and Nuss, 1992). Treatment with HV conidia has been shown to cause hypovirus transmission in other hypovirulent studies (Sciblia and Shain, 1989; Anagnostakis et al., 1998; Root et al., 2005). The spermatization treatments employed by Anagnostakis et al. (1998) and Root et al. (2005) also caused virus acquisition, but at lower rates than demonstrated in this study. In their studies, 
conidial treatments were applied in a liquid solution, not as an agar slurry as in this experiment. Slurry consistency (blended agar medium) may have promoted its persistence on the surface of cankers and subsequent growth of the inoculum strains, thereby increasing the likelihood of hypovirus transmission. Also, cankers were treated three times during the 2004 season, providing a constant source of $\mathrm{HV}$ inoculum which has been shown to increase the likelihood of hypovirus acquisition (Griffin, 1999). Although this slurry method has been used previously for spermatization (Root et al., 2005), bark samples were not collected to analyze hypovirus acquisition by the canker inciting strain.

Hypovirulent isolates were recovered from PI cankers initiated with all wild-type strains isolated from site, even if the strains used to initiate PI cankers failed to acquire the hypovirus via anastomosis under laboratory conditions (Fig. 18). This confirms previous findings that hypovirus acquisition occurs more readily in forest settings than in the laboratory (Double, 1982; Bell, 2004). Laboratory and field settings differ in the amount of time virulent strains are exposed to HV dsRNA sources. Laboratory assays examine hypovirus transmission during a limited amount of time and thus allows for limited hyphal contact for anastomosis to occur. For example, paired cultures can only grow for 12-15 days before both colony margins reach the edge of the Petri dish. During growth in culture, the only site where transmission can occur is at actively growing hyphal tips that are adjacent to one another. Treated field cankers are not under the same time and space limitations and can be converted as long as the hypovirulent treatment inoculum persists on the canker (Griffin, 1999). Milgroom and Cortesi (2004) suggest the use of virus genealogical studies in studying hypovirus transmission dynamics in nature because such analyses integrate more interactions over longer time periods. The viral genomes from infected C. parasitica isolates collected from a population can be compared among host v-c types from 
which the viruses were isolated. Viral sequence similarities then can be used to infer frequency of movement between v-c groups (Carbone et al., 2004). While more in depth than laboratory assays, these genealogical studies are more complicated and time consuming. Laboratory assays presumably only underestimate hypovirus transmission frequencies, and although they vary from field observations, correlations between laboratory and field transmission frequencies have been illustrated (Double, 1982; Hobbins et al., 1992; Balbalian, 1998; Bell, 2004).

The recovery of hypovirulent isolates from the treated infections was similar between cankers painted with TG and CH spermatia, suggesting that TG strains pose no advantage in hypovirus transmission via anastomosis. This would be expected because hypovirus transmission is controlled by vic alleles (Cortesi et al., 2001), which would not presumably be affected during the transformation process. However, assuming equal rates of hypovirus transmission also assumes that $\mathrm{HV}$ isolates recovered from $\mathrm{CH}$ plots acquired their hypovirus via anastomosis and were not introduced strains. Any brown-hypovirulent $(\mathrm{BH})$ isolates recovered would represent the MAT-1 portion of the treatment inoculum applied to canker, since the brown phenotype is a mutant trait not frequently observed in nature. Recovery of orange-hypovirulent $(\mathrm{OH})$ isolates however, may either represent recovery of the MAT-2 portion of the treatment inoculum or converted thallus material. The lack of a hyg ${ }^{\mathrm{R}}$ marker in $\sim 96 \%$ of the $\mathrm{OH}$ isolates recovered from TG plots confirms hypovirus acquisition of the thallus via anastomosis, but no such confirmation is possible with $\mathrm{OH}$ strains isolated from $\mathrm{CH}$ plots. To compare hypovirus transmission between TG and CH strains more rigorously, atypical HV treatment inoculum such as methionine auxotrophic strains (Shain and Miller, 1992) or brown-pigmented strains (Bell, 2004) could be used to distinguish input strains from canker isolates that acquired the hypovirus via anastomosis. 
Hypovirus acquisition by spermatized cankers in TG plots may both promote and hinder biological control. Hypovirus acquisition may retard canker growth, thereby prolonging the life of the tree. The "converted" canker then may act as a source of HV conidia, increasing the pool of HV inoculum needed to establish hypovirulence and overcome the barrier imposed by virulent strains. Hypovirulent cankers, however, do not produce perithecia (Elliston, 1985), so virulent cankers are needed for the production of HVAs in TG plots. If all spermatized cankers acquire hypoviruses from treatment strains and become hypovirulent, HVA production may be hindered considerably. Most spermatized cankers in this study yielded many perithecia after one growing season (Table 9), but the effect that hypovirus acquisition will have on the production of future perithecia is uncertain. A converted canker may grow slowly and cease perithecia production at the boundary of the canker, but the original infected area will presumably remain virulent (Jones, 2004). These virulent areas may continue to produce perithecia that may be spermatized by TG HV conidia produced elsewhere. Even if no new perithecia are produced, HVA-producing perithecia persist on cankers one year following spermatization treatments (Root et al., 2005). In these studies, it is unclear whether the perithecia were the result of the original treatment or a new spermatization event involving persistent HV treatment inoculum from the previous year. A balance between canker cessation and HVA production could ultimately increase biological control by maintaining a constant source of HVA inoculum. Whether the reduced rate of HVA production will negatively impact dissemination of HV strains remains to be seen.

The conidial slurry treatment applied to cankers in this study effectively spermatized PI cankers of MAT-2 mating type. Nineteen of 23 (82.6\%) MAT-2 cankers outcrossed to the MAT-1 treatment strains and produced ascospores displaying the mutant brown pigmentation (calculated from Table 9). This value represents the minimum percentage of cankers that 
outcrossed to the treatment, since it is possible that bark discs were collected from an area of the canker that did not contain outcrossed perithecia, though they may have been present elsewhere on the canker. Likewise, the selection process for perithecia may have missed outcrossed perithecia on bark discs that contained as many as 20-30 perithecia in some cases. Punchinitiated cankers also showed much higher outcrossing rates than natural cankers (16.7\%), presumably because many natural cankers already were spermatized by wild-type conidia. The percentage of perithecia that outcrossed to MAT-1 strains was $68.6 \%$, with the remaining perithecia being accounted for by outcrossing to indigenous conidia or selfing. Spermatization efficacy may have been increased by applying the treatment earlier in the season to artificially spermatize trichogynes before they naturally outcross to wild-type spermatia. Root et al. (2005) and Anagnostakis (1998) found that cankers were more likely to outcross to the treatment strain when treated earlier in the growing season, even when external signs of the canker were not evident.

Hypovirulent ascospore progeny were isolated from all plots where TG inoculum was introduced, though only from cankers spermatized by the transgenic MAT-1 treatment strain (EP146/pxHE7) and at lower than expected rates (Fig. 19). Although hypovirulent ascospores have been produced in Mendelian ratios from transgenic C. parasitica strains under laboratory conditions (Chen et al., 1993), segregation of ascospores from most perithecia did not conform to expected values in a previous field trial that involved a related but different hypovirus, CHV1EP713 (Root et al., 2005). Root et al. (2005) noted that the lack of HVAs may be caused by reduced germination viability of transgenic spores. Alternatively, copies of the cDNA transgene may have been present but not expressed in some of the OV or BV ascospore isolates, thereby skewing morphology ratios in TG plots. Sexually inherited transgenes have been silenced by 
methylation in several ascomycetes (Selker and Garrett, 1988; Goyon and Faugeron, 1989).

Leslie and Dickman (1991) also discovered that single-copy hyg ${ }^{\mathrm{R}}$ genes inserted into Gibberella fujikuroi segregated as expected, but when more than one hyg $^{\mathrm{R}}$ gene was introduced, genes were not inherited at expected ratios. In their study, some ascospore progeny did not phenotypically express hyg ${ }^{\mathrm{R}}$, though Southern blot analyses confirmed the presence of the gene. In the present study, multiple copies of the cDNA gene may have been incorporated into ascospore progeny causing lack of expression, as was suggested in a previous transgenic field release of $C$. parasitica (Root et al., 2005). Furthermore, in this study, morphology ratios from each perithecium were summed before chi-square analyses and the error for each perithecium accumulated in the final ratio, although this accumulation effect was not seen in either the $\mathrm{CH}$ or $\mathrm{V}$ perithecia analyzed. Inheritance rates of the cDNA transgene could be more suitably tested by isolating several more ascospores from single perithecia, since a perithecium arises from the ascogonium of one plasmogamy event (Alexopoulos et al., 1996).

Transgenic strain EP155/pxHE7 failed to produce any morphologically distinct HVAs, though some variant colonies showed hyg ${ }^{\mathrm{R}}$ and faint dsRNA bands. Although hyg ${ }^{\mathrm{R}}$ virulent isolates have been reported in a previous C. parasitica field release (Root et al., 2005), several morphologically distinct hypovirulent isolates also were isolated. Also, hyg ${ }^{\mathrm{R}}$ virulent individuals were collected at low frequencies ( 1 or 2 resistant individuals out of 20 isolates) in this previous study (Root et al., 2005). This varies from the 1:1.6 ratio $\left(\right.$ hyg $^{\mathrm{R}}$ :hyg $\mathrm{h}^{\mathrm{S}}$ ) found for many perithecia tested in this study (Table 11). There are two possible reasons for lack of stable hypovirus inheritance by HVAs: 1) the ascospore progeny may have been resistant to virus infection; or 2) there was a mutation in the cDNA transgene of parental strain EP155/pxHE7 before field application (Bradley Hillman, personal communication). Many of the hyg ${ }^{\mathrm{R}}$ HVA variant 
individuals from a suspected EP155/pxHE7 cross were able to become stably infected with hypoviruses after acquisition via anastomosis (data not shown), suggesting that host resistance was not responsible for lack of stable hypovirus infection. Polymerase chain reaction (PCR) methods have determined the presence of cDNA fragments in these hyg ${ }^{\mathrm{R}}$ virulent strains (Donald Nuss, personal communication). Faint bands of dsRNA may indicate transcription of a mutated transgene without subsequent cytoplasmic dsRNA replication. This mutation or silencing may have occurred during the transformation process, or perhaps the introduced gene was mutated at some point during subculture, as has been found with transgenes in Glomerella cingulata (Rodriguez and Yoder, 1987). A mutation in the RNA polymerase encoding region may have inhibited autonomous dsRNA replication (Nuss, 1992b). This would not have been morphologically detected in a mutated EP155/pxHE7 parent if an infection with an unmutated dsRNA hypovirus already was established. This mutation also may have been responsible for the "pink" morphology of conidia isolated from an EP155/pxHE7 culture (Fig. 22), although this strange morphology was only observed in conidia and only once out of five laboratory conidial isolations. A change in relatively few base pairs of the viral genome can cause a significant difference in culture morphology of the infected host. For example, nucleotide sequences are 90\% similar between CHV1-EP713 and CHV1-Euro7 (Chen and Nuss, 1999), but culture morphology of strains infected with these two viruses is drastically different. Southern blot analyses will confirm the length of the cDNA fragments present in these defective strains and illustrate any possible rearrangements of the transgenic insert.

More than 1000 HVAs were isolated during the course of this study, all from TG plots. Although the role of these HVAs in disease dissemination is unclear, the results of a few studies suggest that virulent ascospores are major contributors to tree-to-tree spread. In the early 1900's, 
researchers demonstrated that ascospores were forcibly expelled and able to be carried by wind currents, which led them to hypothesize their role in long distance dispersal (Rankin, 1912; Heald et al., 1915). More recent studies on v-c type diversity within stands support this longdistance dissemination hypothesis. Since v-c depends on the allelic composition at six loci (Cortesi et al., 2001), allelic recombination during sexual mating can result in ascospores of different v-c types (Anagnostakis, 1977). MacDonald and Double (1978) found a large number of different v-c types within the same $20 \mathrm{~m}$ x $20 \mathrm{~m}$ plots, which they suggested may represent the dissemination and establishment of genetically recombined ascospores. Data from this study were later analyzed using matrix statistics to test randomness of v-c type distribution (Milgroom et al., 1991). Vegetative compatibility types were initially found to be distributed in a nonrandom (i.e. clustered) pattern, but when multiple cankers were eliminated from the same tree, the pattern was found to be random in 97 of the 98 tests (Milgroom et al., 1991). If HVAs are able to initiate infections as has been found for virulent ascospores (Anderson and Rankin, 1914), they may aid in the dissemination and establishment of HV strains.

Hypovirulent ascospores were not only found to increase the long distance biological control potential of $\mathrm{HV}$ inoculum, but they also were capable of transmitting their hypoviruses to 15 v-c types isolated from the site when they were paired under laboratory conditions.

Dissemination of the cDNA transgene to a variety of v-c types has been illustrated previously, both in laboratory HVA production (Chen et al., 1993) and field release trials (Anagnostakis et al., 1998), but this is the first transgenic field release study to demonstrate hypovirus transmission to almost all representative v-c types from the release site. Cortesi et al. (2001) demonstrated that specific vic alleles are more important than others in hypovirus transmission. Using isolates of the eight most common v-c types from the site to artificially establish female 
parent cankers ensured the incorporation of the most common vic alleles into HVA progeny and promoted hypovirus transmission when they were paired with these $15 \mathrm{v}$-c types in the laboratory. As discussed earlier, however, laboratory transmission rates do not always represent what may happen in the field. These laboratory pairings may underestimate the transmission potential of HVAs because hypovirus transmission occurs more readily in nature than in the laboratory (Double, 1982). Also, the vic alleles from three common v-c types used to initiate PI cankers were not represented in HVA progeny because EP155/pxHE7 failed to produce HVAs when they spermatized these common MAT-1 female parents. Establishment of a functional MAT-2 transgenic strain should further increase disseminative potential. Although the presence of $\mathrm{HV}$ isolates representing a variety of v-c types may be promising, the degree to which vegetative incompatibility restricts hypovirus dissemination is unclear (Milgroom and Cortesi, 2004). As this test continues, monitoring hypovirus infection status of cankers and disease development in transgenic plots will provide more information on the role of HVAs representing different v-c types and their role in enhancing biological control.

Hypovirulent ascospores may contribute to HV dissemination in several ways. First, ascospores of virulent $C$. parasitica strains have been shown to cause infections on chestnut trees in the presence of wounds (Anderson and Rankin, 1914). Although such infections have never been demonstrated for HVAs, if HVAs are capable of establishing HV infections, than the number of HV cankers and hence the pool of HV inoculum (in the form of conidia) will increase. Also, since HVAs represent a variety of v-c types (see above), the inoculum produced by HVAestablished cankers also will represent a variety of v-c types, further increasing HV inoculum of various v-c types. A second way HVAs may contribute to HV dissemination is by disseminating to virulent cankers, germinating, and converting the underlying thallus. Although perithecia of 
C. parasitica release thousands of ascospores per hour (Wolf and Wolf, 1947), the number of HVAs that will land on a virulent canker may be low. Even if a small number of HVAs land on a virulent canker, germinate, and transmit their hypovirus to the underlying thallus, it is not clear whether transmission to those few areas would effectively arrest canker growth. A third way in which HVAs may contribute to HV dissemination is by acting as spermatia, which ultimately could increase the number of HVAs produced by other cankers in the stand. Ascospores of $C$. parasitica have never been demonstrated to act as spermatia, and only asexual spore stages of fungi are noted as spermatia by Alexopoulos et al. (1996) and Deacon (1997). Future research should focus on clarifying the role and capability of HVAs.

Conidia are thought to be responsible for local disease spread presumably being vectored by insect and rain water (Garrod et al., 1985; Roane et al., 1986; Diamandis and Perlerou, 2005). This is the first transgenic field release study that has established cankers to produce conidial inoculum. This was done principally by scratch-wounding trees and painting the wounded area with different inoculum types. Wounded areas then were covered with absorptive pads to protect inoculum and promote infection establishment. When absorbent pad coverings were removed one month following inoculation of SI cankers, wounded areas were heavily covered with mycelium and stroma (Fig. 12A). In time, the bark began to exfoliate indicating that the wounding method employed may have been too invasive. By October, 2004 many of the scratch-initiated cankers in TG and CH plots exhibited bark exfoliation and little to no stroma production (Fig. 12B). This lack of canker establishment considerably reduced the pool of HV conidial inoculum. These cankers must be expanded or reestablished to maintain a source of conidial inoculum, or other procedures of conidial release must be explored. 
Despite the presence of stroma on all bark discs collected from SI cankers, no pycnidia could be discerned on samples collected from the $\mathrm{CH}$ plots. Thus it was not possible to compare conidia production between TG and CH cankers. Although high percentages of HV conidia have been reported when transgenic C. parasitica is grown in culture (Chen et al., 1993), this is the first study to illustrate high percentages in nature. These high percentages also illustrate the stability of these transgenic $C$. parasitica strains when introduced into live chestnut trees; a finding that was previously demonstrated for CHV1-EP713 transformants on dormant chestnut bolts (Chen et al., 1993). Most SI cankers analyzed from V plots yielded 100\% V conidia, with the exception of one canker in plot “D.” Pycnidia from this canker yielded approximately 80\% HV conidia, all of which were hyg . This may have been caused by contamination of inoculum before field application, but HV conidia were not isolated in any other V plot. Another possible explanation is the dissemination of HV isolates from nearby transgenic plot "C" and subsequent hypovirus transmission to the isolate used to initiate the SI canker. Hypovirus transmission may have readily occurred since the SI canker strains introduced in each plot were isogenic (i.e. contained identical vic alleles) and differed only by their hypovirus infection status. If the SI canker was indeed converted in this way, it presumably would have produced HV conidia as has been demonstrated previously (Russin and Shain, 1985; Bell, 2004).

The consistently high percentages of HV conidia produced by TG cankers may pose a considerable advantage over $\mathrm{CH}$ strains that often produce conidia at inconsistent rates in the field (Bauman, unpublished data). These conidia most likely will increase the local spread of HV strains by establishing HV cankers and/or converting virulent cankers on nearby stems (Garrod et al., 1985; Hobbins et al., 1992; see below). Also, HV conidia produced by TG strains 
pose a further advantage to HV dissemination because they can spermatize virulent cankers and lead to the formation of HVAs, while conidia produced by $\mathrm{CH}$ strains cannot.

Although this study monitored canker development during only one growing season, there were some noteworthy observations of inoculum dissemination. Hypovirulent isolates were recovered from two unpainted natural cankers on SI trees (one in a TG plot and one in a V plot). Recovery of $\mathrm{HV}$ isolates from a canker that was not treated indicates dissemination of an HV strain from a treated canker and subsequent hypovirus transmission. Two separate unpainted cankers (one in a TG plot and one in a V plot) on SI trees also had perithecia that yielded brown pigmented spore colonies. These two cankers were not treated with spermatia however, and the only way they could have outcrossed to a brown treatment strain is if brown inoculum disseminated from a treated canker (either SI or PI). These results most likely indicate that conidia from SI cankers, or the inocula used to initiate these cankers, washed down the stem and transmitted their hypovirus or spermatized natural cankers below. Previous studies have demonstrated the movement of HV conidia down tree stems and subsequent hypovirus transmission to cankers below (Garrod et al., 1985; Hobbins et al., 1992). Another indication of dissemination was the recovery of $\mathrm{HV}$ isolates from a V plot where no $\mathrm{HV}$ strains were introduced. Hypovirulent bark isolates were recovered at varying rates from two natural cankers and two SI cankers in a V plot (Table 7), and 80\% HV conidia were isolated from an SI canker in the same plot (Fig. 21). All HV isolates recovered from V plots were isolated in plot “D,” and they were all hyg ${ }^{S}$. Virulent plot “D” is relatively close to TG plot “C” ( 55 ft), perhaps facilitating the dissemination of HV inoculum and subsequent hypovirus transmission. The sensitivity of HV isolates from plot " $\mathrm{D}$ ” to hygromycin supports this hypothesis, since hyg ${ }^{\mathrm{R}}$ is a nuclear encoded trait and is not transmitted via anastomosis (Choi and Nuss, 1992). 
Dissemination from a TG plot to a nearby V plot is somewhat unexpected since no tree-to-tree dissemination was detected within any of the TG plots. However, the dissemination of HV isolates to a V control plot also has been seen in previous C. parasitica field studies (MacDonald and Double, unpublished data). Whether conidia or ascospores were responsible for this dissemination is unclear at this time.

The intent of this transgenic study was to use a mildly debilitating hypovirus (CHV1Euro7) to increase hypovirus establishment by increased inoculum production of the HV strains when compared to the highly debilitating hypovirus (CHV1-EP713) used in past field releases. In previous non-transgenic studies, CHV1-Euro7-infected strains were found to cause larger cankers with more stroma production when compared to CHV1-EP713 strains (Chen and Nuss, 1999; Double, unpublished data). However, a recent field study found no difference in canker size and only slightly greater sporulation of CHV1-Euro7 strains when compared to isogenic strains infected with the CHV1-EP713 hypovirus (Bauman, unpublished data). Also, outcrossing rates were similar between perithecia collected for the present study and a previous experiment utilizing the transgenic CHV1-EP713 strain (Double, unpublished data). Any advantage provided by strains infected with CHV1-Euro7 is yet to be seen in the present study. More time is needed to determine whether the hypovirus utilized in this transgenic release will lead to better biological control than the CHV1-EP713 hypovirus used in the two previous transgenic field releases (Anagnostakis et al., 1998; Root et al., 2005). Autonomous establishment of HV cankers and subsequent inoculum production could be evidence of increased biological control potential provided by less-debilitating hypovirus CHV1-Euro7.

Even though this study is its initial phase, the data on spore production support the hypothesis that transgenic strains provide increased biological control potential. The production 
of HVAs and their increased capability to transmit hypoviruses via anastomosis may have a significant impact on virus dissemination. However, the factors that contribute to hypovirus dissemination in forest settings are poorly understood. For example, saprophytic C. parasitica growing on dead trees and wood piles may act as a significant source of sexual and asexual inoculum, but their contributions to hypovirus dissemination have not been considered until recently (Prospero et al., unpublished data). Also, transgenic strains may not be able to overcome some of the barriers to hypovirus dissemination. High elevations, drought stress, and hardwood competition have been attributed to superficial canker instability, presumably because of the stresses they impose on host trees (Griffin et al., 1991; Griffin et al., 1993). Even if transgenic strains overcome the major barriers to dissemination, the presence of a virus-encoding gene is not advantageous for the fungus and may be highly selected against in nature (Milgroom and Cortesi, 2004). Monitoring disease development in the upcoming years will provide key information on the fate of transgenic inoculum being produced and whether or not it promotes establishment of hypovirulent strains and biological control of chestnut blight. 


\section{Summary}

1.) None of the inoculation treatments had a significant effect on tree growth as measured by DBH and ratings of tree health after the first treatment season.

2.) The bark scratch method used to initiate cankers resulted in exfoliating bark and did not produce as much HV inoculum as intended in the TG and CH plots. Some scratchinitiated (SI) cankers in V plots also exfoliated, but overall SI cankers in V plots expanded significantly more than cankers in the TG and CH plots.

3.) Cankers in the cytoplasmic hypovirulent $(\mathrm{CH})$ plots were rated as having the least stroma and most callus, followed by cankers in the virulent (V), and then transgenic (TG) plots. Differences in ratings may be explained in part by differences in rater subjectivity.

4.) Hypovirulent (HV) isolates were recovered from $~ 40 \%$ of the bark plugs removed from treated cankers in both $\mathrm{CH}$ and TG plots. Ninety-six percent of the HV isolates recovered from TG plots were hygromycin-sensitive $\left(\mathrm{hyg}^{\mathrm{S}}\right)$, which indicates that the hypovirus was transmitted to canker thallus via hyphal anastomosis.

5.) Hypovirulent ascospore (HVA) isolates were recovered only from TG plots, but at lower than expected Mendelian ratios.

6.) The brown-pigmented MAT-1 treatment strain (EP146-based strains) effectively spermatized $93.3 \%, 75 \%$, and $50 \%$ of the punch-initiated (PI) cankers sampled in TG, $\mathrm{CH}$, and $\mathrm{V}$ plots respectively.

7.) The cDNA transgene was transmitted to ascospores representing at least $15 \mathrm{v}-\mathrm{c}$ types different from parental strains.

8.) No morphologically-distinct HVA isolates were recovered from TG cankers spermatized by the MAT-2 treatment strain EP155/pxHE7, although 71.4\% of these cankers contained hygromycin-resistant $\left(\right.$ hyg $\left.^{\mathrm{R}}\right)$ isolates. These hyg ${ }^{\mathrm{R}}$ isolates contained the cDNA transgene (determined by PCR), but did not contain dsRNA.

9.) Pycnidia (asexual fruiting bodies) producing viable conidia only were collected from SI trees in TG and V plots, which precluded comparison of rates of hypovirus transmission between $\mathrm{CH}$ and TG strains. The average $\mathrm{HV}$ conidial inheritance rate of SI cankers was 98.6\% in TG plots. In V plots, three of the four SI cankers analyzed yielded $0 \% \mathrm{HV}$ conidia, but one canker yielded $\sim 80 \%$. 


\section{$\underline{\text { References }}$}

Alexopoulos, C.J., Mims, C.W., Blackwell, M. 1996. Introductory Mycology: $4^{\text {th }}$ edition. John Wiley \& Sons, Inc. New York City, NY. 869 p.

Amsellem, Z., Cohen, B.A., Gressel, J. 2002. Engineering hypervirulence in a mycoherbicidal fungus for efficient weed control. Nature Biotechnology 20: 1035-1039.

Anagnostakis, S.L. 1977. Vegetative incompatibility in Endothia parasitica. Experimental Mycology 1: 306-316.

Anagnostakis, S.L. 1979. Sexual reproduction of Endothia parasitica in the laboratory. Mycologia 71: 213-215.

Anagnostakis, S.L. 1982. Biological control of chestnut blight. Science 215: 466-471.

Anagnostakis, S.L. 1983. Conversion to curative morphology in Endothia parasitica and its restriction by vegetative compatibility. Mycologia 75: 777-780.

Anagnostakis, S.L. 1984. Nuclear gene mutations in Endothia (Cryphonectria) parasitica that affect morphology and virulence. Phytopathology 74: 561-565.

Anagnostakis, S.L. 1987. Chestnut blight: The classical problem of an introduced pathogen. Mycologia 79: 23-37.

Anagnostakis, S.L. 1998. Introduction of new genotypes into ascospore progeny of Cryphonectria parasitica in the field. Mycological Research 102: 685-686.

Anagnostakis, S.L., Chen, B., Geletka, L.M., Nuss, D.L. 1998. Hypovirus transmission to ascospore progeny by field-released transgenic hypovirulent strains of Cryphonectria parasitica. Phytopathology 88: 598-604.

Anagnostakis, S.L., Day, P.R. 1979. Hypovirulence conversion in Endothia parasitica. Phytopathology 69:1226-1229.

Anagnostakis, S.L., Hau, B., Kranz, J. 1986. Diversity of vegetative compatibility groups of Cryphonectria parasitica in Connecticut and Europe. Plant Disease 70: 536-538.

Anagnostakis, S.L., Jaynes, R.A. 1973. Chestnut blight control: use of hypovirulent cultures. Plant Disease Reporter 57: 225-226.

Anderson, P.J., Anderson, H.W. 1912. The chestnut blight fungus and related saprophyte. Phytopathology 2: 204-210.

Anderson, P.J., Rankin, W.H. 1914. Endothia canker of chestnut. Cornell University Agricultural Experiment Station Bulletin No. 347. 
Arie, T., Christiansen, S. K., Yoder, O. C., and Turgeon, B. G. 1997. Efficient cloning of ascomycete mating type genes by PCR amplification of the conserved MAT HMG box. Fungal Genetics and Biology 21:118-130.

Balbalian, C.J. 1998. The influence of vegetative incompatibility genes on the transmission of hypoviruses between strains of Cryphonectria parasitica. Master's Thesis. West Virginia University.

Barr, M.E. 1978. The Diaporthiales in North America. Mycologia Memoir No. 7. J. Cramer. Lehre, Germany. 232 p.

Bell, B.C. 2004. Comparison of chestnut canker treatment procedures for hypovirus introduction. Master's thesis. West Virginia University.

Bissegger, M., Rigling, D., Heiniger, U. 1997. Population structure and disease development of Cryphonectria parasitica in European chestnut forests in the presence of natural hypovirulence. Phytopathology 87: 50-59.

Braun, E.L. 1950. Deciduous forests of eastern North America. The Blackiston Co., Philadelphia, PA. 596 p.

Burnham, C.R., Rutter, P.A., French, D.W. 1986. Breeding blight-resistant chestnuts. Plant Breeding Reviews 4: 347-397.

Carbone, I., Liu, Y.C., Hillman, B.I., Milgroom, M.G. 2004. Recombination and migration of Cryphonectria hypovirus 1 as inferred from gene genealogies and the coalescent. Genetics 166: 1611-1629.

Chen, B., Chen, C., Bowman, B.H., Nuss, D.L. 1996. Phenotypic changes associated with wildtype and mutant RNA transfection of plant pathogenic fungi phylogenetically related to Cryphonectria parasitica. Phytopathology 86:301-310.

Chen, B., Choi, G.H., Nuss, D.L. 1993. Mitotic stability and nuclear inheritance of integrated viral cDNA in engineered hypovirulent strains of the chestnut blight fungus. EMBO J. 12: 2991-2998.

Chen, B., Choi, G.H., Nuss, D.L. 1994. Attenuation of fungal virulence by synthetic infectious hypovirus transcripts. Science 264: 1762-1764.

Chen, B., Nuss, D.L. 1999. Infectious cDNA clone of hypovirus CHV1-Euro7: a comparative virology approach to investigate virus-mediated hypovirulence of the chestnut blight fungus Cryphonectria parasitica. Journal of Virology 73: 985-992.

Choi, G.H., Nuss, D.L. 1992. Hypovirulence of chestnut blight fungus conferred by an infectious viral cDNA. Science 257: 800-803. 
Cortesi, P., Milgroom, M.G. 1998. Genetics of vegetative incompatibility in Cryphonectria parasitica. Applied and Environmental Microbiology 64: 2988-2994.

Cortesi, P., McCulloch C.E., Song H., Lin H., Milgroom. M.G. 2001. Genetic control of horizontal virus transmission in the chestnut blight fungus, Cryphonectria parasitica. Genetics 159: 107-118.

Day, P.R., Dodds, J.A., Elliston, J.E., Jaynes, R.A., Anagnostakis, S.L. 1977. Double-stranded RNA in Endothia parasitica. Phytopathology 67: 1393-1396.

Dawe, A.L., Nuss, D.L. 2001. Hypoviruses and chestnut blight: exploiting viruses to understand and modulate fungal pathogenesis. Annual Review of Genetics 35: 1-29.

Deacon, J.W. 1997. Modern Mycology $3^{\text {rd }}$ Edition. Blackwell Science Ltd. London, U.K. 303 p.

Diamandis, S., Perlerou, C. 2005. The role of Spulerina simploniella in the spread of chestnut blight. Forest Pathology 35: 315-320.

Double, M.L. 1982. The ability of hypovirulent isolates and mixtures of hypovirulent isolates to control artificially established virulent cankers. Pages 145-152 in Proceedings of USDA Forest Service American Chestnut Cooperators’ Meeting. H.C. Smith and W.L. MacDonald, eds. West Virginia University Books, Morgantown.

Elliston, J.E. 1978. Pathogenicity and sporulation of normal and diseased strains of Endothia parasitica in American chestnut. Pages 95-100 in Proceedings of the American Chestnut Symposium. W.L. MacDonald, F.C. Cech, J. Luchok, and C. Smith, eds. West Virginia University Books, Morgantown.

Elliston, J.E. 1985. Characteristics of dsRNA-free and dsRNA-containing strains of Endothia parasitica in relation to hypovirulence. Phytopathology 75: 151-158.

Ellingboe, A. 1992. Breeding blight resistant American chestnut. Pages 47-51 in Proceedings. of the International Chestnut Conference. W.L. MacDonald, M.L. Double, eds. West Virginia University Books, Morgantown.

Fulbright, D.W. 1984. Effect of eliminating dsRNA in hypovirulent Endothia parasitica. Phytopathology 74: 722-724.

Fulbright, D.W., Weidlich, W.H., Haufler, K.Z., Thomas, C.S., Paul, C.P. 1983. Chestnut blight and recovering American chestnut trees in Michigan. Canadian Journal of Botany 61: 3164-3171.

Fulbright, D.W., Paul, C.P., Garrod, S.W. 1988. Hypovirulence: a natural control of chestnut blight. Pages 121-138 in: Biocontrol of Plant Diseases. K.G. Mukerji, K.L. Garg, eds. CRC Press, Boca Raton, FL. 
Garrod, S.W., Fulbright, D.W., Ravenscroft, A.V. 1985. Dissemination of virulent and hypovirulent forms of a marked strain of Endothia parasitica in Michigan. Phytopathology 75: 533-538.

Goyon, C., and G. Faugeron. 1989. Targeted transformation of Ascobolus immersus and de novo methylation of the resulting duplicated DNA sequences. Molecular and Cellular Biology 9: 2818-2827.

Griffin, G.J. 1986. Chestnut blight and its control. Horticultural Reviews 8: 291-336.

Griffin, G.J. 1999. Frequencies and spatial patterns of white hypovirulent and pigmented strains of Cryphonectria parasitica within blight-controlled cankers on grafted American chestnut trees 15-16 years after inoculation. European Journal of Forest Pathology 29: 377-390.

Griffin, G.J. 2000. Blight control and restoration of the American chestnut. Journal of Forestry 98: 22-27.

Griffin G.J., Kahn M.A., Griffin S.L. 1993. Superficial canker instability during winter and virulence of Endothia parasitica associated with managed forest clearcut and plantation American chestnut trees. Canadian Journal of Plant Pathology 15:159-167.

Griffin G.J., Smith H.C., Dietz A., Elkins J.R. 1991. Importance of hardwood competition to American chestnut survival, growth, and blight development in forest clearcuts. Canadian Journal of Botany 69:1804-1809.

Heald, F.D., Gardner, M.W., Studhalter, R.D. 1915. Air and wind dissemination of ascospores of the chestnut-blight fungus. Journal of Agricultural Resources 3: 493-531.

Hebard, F.V. 1982. Biology of virulent and hypovirulent Endothia parasitica on American chestnut (Castanea dentata). Ph.D. thesis, Virginia Polytechnic Institute and State University, Blacksburg. 295 p.

Hebard, F.V. 2002. Meadowview notes 2001-2002. The Journal of The American Chestnut Foundation 16: 7-18.

Hebard, F.V., Griffin, G.J., Elkins, J.R. 1984. Developmental histopathology of cankers incited by hypovirulent and virulent isolates of Endothia parasitica on susceptible and resistant chestnut trees. Phytopathology 74: 140-149.

Heiniger, U., Rigling, D. 1994. Biological control of chestnut blight in Europe. Annual Review of Phytopathology 32: 581-599.

Hepting, G.H. 1974. Death of the American chestnut. Journal of Forest History 18: 60-67. 
Hillman, B.I., Fulbright, D.W., Nuss, D.L., van Alfen, N.K. 2000. Hypoviridae. Pages. 515520 in Seventh Report of the International Committee for the Taxonomy of Viruses. M.H.V van Regenmortel, C.M. Fauquet, D.H.L. Bishop, E.B. Carstens, M.K. Estes, S.M. Lemon, J. Maniloff, M.A. Mayo, D.J. McGeoch, C.R. Pringle et al. Academic Press, San Diego, California.

Hillman, B.I., Suzuki, N. 2004. Viruses of the chestnut blight fungus, Cryphonectria parasitica. Advances in Virus Research 63: 423-472.

Hobbins, D.L., Double, M.L., Sypolt, C.R., MacDonald, W.L. 1992. Interactions between artificially established virulent Cryphonectria parasitica cankers and sources of virulent and hypovirulent inoculum on American chestnut stems. Pages 156-160 in Proceedings of the International Chestnut Conference. W.L. MacDonald, M.L. Double, eds. WV Univ. Press, Morgantown. 215 p.

Jaynes, R.A., DePalma, N.K. 1982. Attempts to control chestnut blight with slurry and conidial sprays of hypovirulent strains. Pages 128-133 in Proceedings of the USDA Forest Service American Chestnut Cooperators’ Meeting. H.C. Smith and W.L. MacDonald, eds. West Virginia University Books, Morgantown.

Jaynes, R.A., Elliston, J.E. 1978. Control of Endothia parasitica cankers on American chestnut sprouts with hypovirulent strains. Pages 110-114 in Proceedings of the American Chestnut Symposium. W.L. MacDonald, F.C. Cech, J. Luchok, and C. Smith, eds. WV Univ. Books, Morgantown. 122 p.

Jaynes, R.A., Elliston, J.E. 1980. Pathogenicity and canker control by mixtures of hypovirulent strains of Endothia parasitica in American chestnut. Phytopathology 70: 453-456.

Jaynes, R.A., Elliston, J.E. 1982. Hypovirulent isolates of Endothia parasitica associated with large American chestnut trees. Plant Disease 66: 769-772.

Jones, W.E. 2004. Recovery of hypovirulent isolates from younger and older Cryphonectria parasitica mycelium in cultures and cankers. Master's Thesis. West Virginia University.

Kuhlman, E.G. 1978. The devastation of American chestnut by blight. Pages 1-3 in Proceedings of the American Chestnut Symposium. W.L. MacDonald, F.C. Cech, J. Luchok, and C. Smith, eds. WV Univ. Books, Morgantown. 122 pgs.

Leslie, J.F., Dickman, M.B. 1991. Fate of DNA encoding hygromycin resistance after meiosis in transformed strains of Gibberella fujikuroi (Fusarium moniliforme). Applied and Environmental Microbiology 57: 1423-1429.

Lilly, V.G., Barnett, H.L. 1951. Physiology of the Fungi. McGraw-Hill Book Company, Inc. New York, NY. 464p. 
Liu, Y.C., Cortesi, P., Double, M.L., MacDonald, W.L., Milgroom, M.G. 1996. Diversity and multilocus genetic structure in populations of Cryphonectria parasitica. Phytopathology 86: 1344-1351.

Liu, Y.C., Durrette, R., Milgroom, M.G. 2000. A spatially-structured stochastic model to simulate heterogeneous transmission of viruses in fungal populations. Ecological Modeling 127: 291-308.

MacDonald, W.L., Double, M.L. 1978. Frequency of vegetative compatibility types in two areas of West Virginia. Pages 103-105 in Proceedings of the American Chestnut Symposium. W.L. MacDonald, F.C. Cech, J. Luchok, and C. Smith, eds. WV Univ. Books, Morgantown. 122 pgs.

MacDonald, W.L., Fulbright, D.W. 1991. Biological control of chestnut blight: use and limitations of transmissible hypovirulence. Plant Disease 75: 656-661.

Marra, R.E. 1998. Selfing in the context of self-incompatibility: the mixed-mating system of the fungus Cryphonectria parasitica. Ph.D. Dissertation. Cornell University.

Melzer, M.S., Dunn, M., Zhou, T., Boland, G.J. 1997. Assessment of hypovirulent isolates of Cryphonectria parasitica for potential in biological control of chestnut blight. Canadian Journal of Plant Pathology 19: 69-77.

Milgroom, M.G. 1995. Population biology of the chestnut blight fungus, Cryphonectria parasitica. Canadian Journal of Botany 73(Suppl.): S311-S319.

Milgroom, M.G., Cortesi, P. 1999. Analysis of population structure of the chestnut blight fungus based on vegetative incompatibility genotypes. Proceedings of the Natural Academy of Science 96: 10518-10523.

Milgroom, M.G., Cortesi, P. 2004. Biological control of chestnut blight with hypovirulence: A critical analysis. Annual Review of Phytopathology 42: 311-338.

Milgroom, M.G., MacDonald, W.L., Double, M.L. 1991. Spatial pattern analysis of vegetative compatibility groups in the chestnut blight fungus, Cryphonectria parasitica. Canadian Journal of Botany 69:1407-1413.

Milgroom, M.G., Wang, K., Yang, Z., Lipari, S., Kaneko, S. 1996. Intercontinental population structure of the chestnut blight fungus, C. parasitica. Mycologia 88:179-190.

Mittempergher, L. 1978. The present status of chestnut blight in Italy. Pages 34-37 in: Proceedings of the American Chestnut Symposium. W.L. MacDonald, F.C. Cech, J. Luchok, and C. Smith, eds. WV Univ. Books, Morgantown. 122 p.

Moffitt, E.M., Lister, R.M. 1975. Application of a serological screening test for detecting double-stranded RNA mycoviruses. Phytopathology 65: 851-859. 
Morris, T.J., Dodds, J.A. 1979. Isolation and analysis of double-stranded RNA from virusinfected plant and fungal tissue. Phytopathology 69: 854-858.

Murrill, W.A. 1906. A new chestnut disease. Torreya 6:186-189.

Newhouse, J.R. 1990. Chestnut blight. Scientific American 263:106-111.

Nuss, D.L. 1992a. Implications of engineered transmissible hypovirulence for biological control of chestnut blight. Pages 3-7 in Proceedings of the International Chestnut Conference. W.L. MacDonald, M.L. Double, eds. WV Univ. Press, Morgantown. 215 p.

Nuss, D.L. 1992b. Biological control of chestnut blight: an example of virus-mediated attenuation of fungal pathogenesis. Microbiological Reviews 56: 561-576.

Nuss, D.L., Koltin, Y. 1990. Significance of dsRNA in plant pathogenic fungi. Annual Review of Phytopathology 28:37-58.

Powell, W.A. 1995. Vegetative incompatibility and mycelial death of Cryphonectria parasitica detected with a $\mathrm{pH}$ indicator. Mycologia 87: 738-741.

Rankin, H.W. 1912. The chestnut canker disease. Phytopathology 2: 99.

Roane, M.K., Griffin, G.J., Elkins, J.R. 1986. Chestnut Blight, Other Endothia Diseases, and the Genus Endothia. APS Press. St. Paul, Minnesota. 53 pgs.

Rodriguez, R. J., Yoder, O.C. 1987. Selectable genes for transformation of the fungal plant pathogen Glomerella cingulata f. sp. phaseoli (Colletotrichum lindemuthianum). Gene 54: 73-80.

Ronderos, A. 2000. Where giants once stood: The demise of the American chestnut and efforts to bring it back. Journal of Forestry 98: 10-11.

Root, C., Balbalian, C., Beirman, R., Geletka, L., Anagnostakis, S., Double, M., MacDonald, M., Nuss, D.L. 2005. Multi-seasonal field release and spermatization trials of transgenic hypovirulent strains of Cryphonectria parasitica containing cDNA copies of hypovirus CHV1-EP713. Forest Pathology 35: 277-297.

Russin, J.S., Shain, L. 1983. Insects as carriers of virulent and hypovirulent isolates of Endothia parasitica. (Abstract) Phytopathology 73: 837.

Russin, J.S., Shain, L. 1985. Disseminative fitness of Endothia parasitica containing different agents for cytoplasmic hypovirulence. Canadian Journal of Botany 63: 54-57.

Sciblia, K.L., Shain, L. 1989. Protection of American chestnut with hypovirulent conidia of Cryphonectria parasitica. Plant Disease 73:840-843. 
Selker, E. U., and Garrett, P.W. 1988. DNA sequence duplications trigger gene inactivation in Neurospora crassa. Proceedings of the Natural Academy of Sciences USA 85:6870-6874.

Shain, L., Miller, J.B. 1992. Movement of cytoplasmic hypovirulence agents in chestnut blight cankers. Canadian Journal of Botany 70:557-561.

Sharf, C.S., DePalma, N.K. 1981. Birds and mammals as vectors of the chestnut blight fungus (Endothia parasitica). Canadian Journal of Zoology 59: 1647-1650.

Sharma, H.C., Sharma, K.K., Seetharama, N., Crouch, J.H. 2003. The utility and management of transgenic plants with Bacillus thuringiensis genes for protection from pests. Journal of New Seeds 5: 53-76.

Shear, C.L., Stevens, N.E. 1916. The discovery of the chestnut blight parasite (Endothia parasitica) and other chestnut fungi in Japan. Science 43: 173-176.

Tang, W., Tian, Y. 2003. Transgenic loblolly pine (Pinus taeda L.) plants expressing a modified delta-endotoxin gene of Bacillus thuringiensis with enhanced resistance to Dendrolimus punctatus Walker and Crypyothelea formosicola Staud. Journal of Experimental Botany 54: 835-844.

Van Alfen, N.K., Jaynes, R.A., Anagnostakis, S.L., Day, P.R. 1975. Chestnut blight: biological control by transmissible hypovirulence in Endothia parasitica. Science 189: 890-891.

Willey, R.L. 1982. Natural dissemination of artificially inoculated hypovirulent strains of Endothia parasitica. Pages 117-127 in: Proceedings of the USDA Forest Service Chestnut Cooperators’ Meeting. H.C. Smith and W.L. MacDonald, eds. West Virginia University Books, Morgantown.

Wolf, F. A., Wolf, T. A. 1947. The Fungi, Vol. II. John Wiley \& Sons, Inc. London, U.K. 538p.

Yang, M.S., Lang, H.Y., Gao, B.J., Wang, J.M., Zheng, J.B. 2003. Insecticidal activity and transgene expression stability of transgenic hybrid poplar clone 741 carrying two insectresistant genes. Silvae Genetica 52: 197-201. 


\section{Appendix A: Growth media used}

Glucose Yeast Extract (GYE; amended from Lilly and Barnett, 1951)

$\begin{array}{ll}\text { Glucose } & 10.0 \mathrm{~g} \\ \text { Yeast Extract } & 2.0 \mathrm{~g} \\ \mathrm{KH}_{2} \mathrm{PO}_{4} & 1.0 \mathrm{~g} \\ \mathrm{Mg}_{2} \mathrm{SO}_{4} & 0.5 \mathrm{~g} \\ \text { Thiamine (Stock) } & 1.0 \mathrm{ml}(0.1 \mathrm{mg} / \mathrm{ml}) \\ \text { Biotin (Stock) } & 1.0 \mathrm{ml} \mathrm{(5} \mathrm{g/ml)} \\ \text { Microelements } & 2.0 \mathrm{ml} \\ & 0.2 \mathrm{mg} \mathrm{Fe} \\ & 0.2 \mathrm{mg} \mathrm{Zn}{ }^{2+} \\ & 0.1 \mathrm{mg} \mathrm{Mn}{ }^{2+} \\ \text { Agar } & 20.0 \mathrm{~g} \\ \text { Milli-Q Water } & 1000.0 \mathrm{ml}\end{array}$

Potato Dextrose Agar Amended with Methionine and Biotin (PDAmb; Anagnostakis, 1977)

$\begin{array}{ll}\text { PDA (DIFCO) } & 39.0 \mathrm{~g} \\ \text { 1-1 Methionine } & 0.1 \mathrm{~g} \\ \text { Biotin } & 1.0 \mathrm{ml} \\ \text { Milli-Q Water } & 1000.0 \mathrm{ml}\end{array}$

Brom Cresol Green (BCG; Powell, 1995)

$\begin{array}{ll}\text { Glucose } & 10.0 \mathrm{~g} \\ \text { Malt Extract } & 7.0 \mathrm{~g} \\ \text { Yeast Extract } & 2.0 \mathrm{~g} \\ \text { Tannic Acid } & 0.8 \mathrm{~g} \\ \text { Brom Cresol Green } & 0.05 \mathrm{~g} \\ \text { Agar } & 15 \mathrm{~g} \\ \text { Tween } & 12 \mathrm{drops} \\ \text { Milli-Q Water } & 1000.0 \mathrm{ml}\end{array}$




\section{Appendix B: Modified dsRNA extraction protocol}

Cellophane (Flexal Corp.) was cut to the diameter of $7 \mathrm{~cm}$, added to a glass Petri dish containing tap water, covered, and autoclaved for 24 minutes at 15 psi. The cellophane then was aseptically transferred to Petri dishes containing GYE/A media (Appendix A). Agar plugs containing mycelium of test isolates were grown on four of these GYE/A plates overlaid with cellophane. Plates were incubated at $20^{\circ} \mathrm{C}$ for $7-10$ days. The mycelium then was scraped from the cellophane into a cold mortar, immersed in liquid nitrogen and ground to a fine powder.

The mycelial powder from the above step was added to a $30 \mathrm{ml}$ polyallomer screw cap test tube, to which was added: $10 \mathrm{ml} 2 \mathrm{X}$ STE (containing 20\% sodium dodecyl sulfate), $11 \mathrm{ml}$ phenol (containing $0.1 \%$ 8hydroxyquinoline) and $8 \mathrm{ml}$ chloroform / isoamyl alcohol (24:1). The tubes were capped, covered with ice and placed on a rotary shaker ( $\sim 100 \mathrm{rpm}$ ) for 35 minutes. After shaking, the tubes were centrifuged for 30 minutes at $8,000 \mathrm{rpm}$ at $0-4^{\circ} \mathrm{C}$. The aqueous phase was collected in a $25 \mathrm{ml}$ graduated cylinder and the volume was brought up to $20 \mathrm{ml}$ with $1 \mathrm{X}$ STE. Ethanol (95\%) then added until a final concentration of 15\%.

Twelve grams of chromatographic cellulose powder (Whatman CF-11 cellulose) was equilibrated with 200 $\mathrm{ml} \mathrm{STE}: 15 \%$ EtOH. The cellulose solution was mixed continuously and $25 \mathrm{ml}$ was added to fritted glass columns and allowed to drain. The nucleic acid sample was then applied to the column and washed with 80-100 ml STE:15\% EtOH. Bromphenol blue solution was added to the surface of the CF-11 column (3-5 drops) then nucleic acids were eluted with 11ml $1 \mathrm{X}$ STE (no alcohol). Eluted acids were collected in a $30 \mathrm{ml}$ glass Corex tube.

Eighteen $\mathrm{ml}$ of $95 \% \mathrm{EtOH}$ and 9 drops of 3M sodium acetate were added to each tube. The tubes were covered with parafilm and the contents were mixed thoroughly. The samples were stored at $-20^{\circ} \mathrm{C}$ overnight. Twenty-four hours later, the tubes were removed from the freezer and centrifuged at $8000 \mathrm{rpm}$ for 30 minutes. The supernatant then was decanted and the excess alcohol was wiped from the tubes along the line of centrifugation with kimwipes. The dsRNA precipitate was resuspended with $1 \mathrm{ml}$ of the resuspension buffer and vortexed for 30 seconds.

Traces of DNA were degraded in the nucleic acid sample by adding $100 \mu \mathrm{l} 0.5 \mathrm{M} \mathrm{MgCl} 2$ and $20 \mu \mathrm{L}$ DNAse (Promega RQ1 RNAse-free DNAse) for 60 minutes. Two $\mathrm{ml}$ of cold 95\% EtOH and 1 drop of sodium acetate were then added to each tube. The solution was vortexed and the tubes were stored at $-20^{\circ} \mathrm{C}$ for 2 hours. Samples were centrifuged at 14,000 rpm and alcohol was decanted. The remaining pellet was dried and resuspended in $15 \mu \mathrm{l}$ resuspension buffer. The nucleic acid suspension then was run for two hours at $100 \mathrm{mV}$ on an agarose (1\%) gel stained with ethidium bromide. Gels were examined under UV light $(250 \mathrm{~nm})$ for florescent bands of dsRNA and photographed with Genesnap software (Syngene $\left.{ }^{\circledR}\right)$.

\section{Buffers for dsRNA extractions:}

10X STE:

$0.5 \mathrm{M}$ Tris $61.0 \mathrm{~g}$

0.1 M Sodium chloride $58.0 \mathrm{~g}$

0.001M Disodium EDTA $3.7 \mathrm{~g}$

Distilled Water $1000.0 \mathrm{ml}$

$\mathrm{pH}$ adjusted to 6.8 with glacial acetic acid

10X TBE

Tris $54.5 \mathrm{~g}$

Boric Acid $27.8 \mathrm{~g}$

Disodium EDTA $1.9 \mathrm{~g}$

Distilled Water $1000.0 \mathrm{ml}$

\author{
Bromcresol Blue Solution \\ Bromcresol Blue $50.0 \mathrm{mg}$ \\ Sucrose $25.0 \mathrm{~g}$ \\ Distilled Water $50.0 \mathrm{ml}$
}

\section{Resuspension Buffer}

10X TBE $2.0 \mathrm{ml}$

Sucrose $4.0 \mathrm{~g}$

Distilled Water $38.0 \mathrm{ml}$ 


\section{Appendix C: ANOVA tables from statistical analyses}

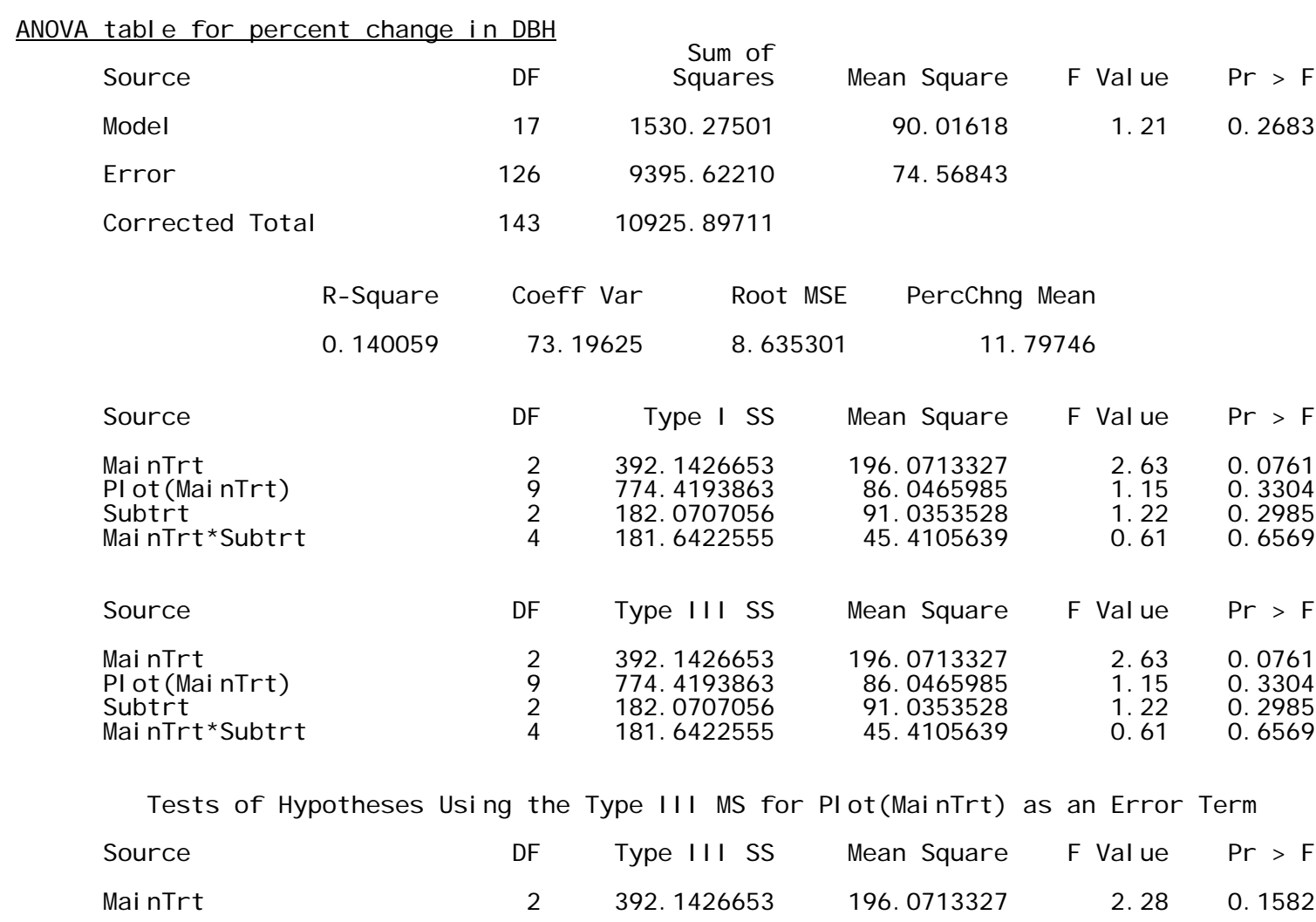

ANOVA table for sI canker expansion

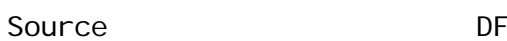

Model

Error

Corrected Total
DF

26

Sum of
Squares

110694.5100

69

21271.2394

95

131965.7493
Mean Square $F$ Value $P r>F$
4257.4812
13.81
$<.0001$
308.2788

Arealnc Mean

23. 12240

\begin{tabular}{|c|c|c|c|c|c|}
\hline Source & DF & Type I SS & Mean Square & F Value & $\mathrm{Pr}>\mathrm{F}$ \\
\hline $\begin{array}{l}\text { Maintrt } \\
\text { PI ot ( Maintrt) } \\
\text { Tree(PIot) } \\
\text { Strai n }\end{array}$ & $\begin{array}{r}2 \\
9 \\
12 \\
1\end{array}$ & $\begin{array}{r}74600.70443 \\
24712.14648 \\
7089.61022 \\
1146.43815\end{array}$ & $\begin{array}{r}37300.35221 \\
2745.79405 \\
590.80085 \\
1146.43815\end{array}$ & $\begin{array}{r}121.00 \\
8.91 \\
1.92 \\
3.72\end{array}$ & 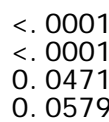 \\
\hline
\end{tabular}




\begin{tabular}{|c|c|c|c|c|c|c|c|}
\hline Maintrt*Strain & 2 & 3145.61068 & 1572.80534 & & & 5.10 & 0.0086 \\
\hline Source & DF & Type III SS & Mean Square & & $\mathrm{F}$ & Val ue & $\mathrm{Pr}>\mathrm{F}$ \\
\hline $\begin{array}{l}\text { Maint rt } \\
\text { PI ot (Maintrt) } \\
\text { Tree(PIot) } \\
\text { Strai n } \\
\text { Maintrt*Strain }\end{array}$ & $\begin{array}{r}2 \\
9 \\
12 \\
1 \\
2\end{array}$ & $\begin{array}{r}17219.22428 \\
12410.78504 \\
7089.61022 \\
1146.43815 \\
3145.61068\end{array}$ & $\begin{array}{r}8609.61214 \\
1378.97612 \\
590.80085 \\
1146.43815 \\
1572.80534\end{array}$ & & & $\begin{array}{r}27.93 \\
4.47 \\
1.92 \\
3.72 \\
5.10\end{array}$ & $\begin{array}{l}<.0001 \\
0.00001 \\
0.0471 \\
0.0579 \\
0.0086\end{array}$ \\
\hline Tests of Hypotheses Using & the & Type III MS for & PI ot ( Maintrt) & as & an & Error & Term \\
\hline Source & DF & Type III SS & Mean Square & & $\mathrm{F}$ & Val ue & $\mathrm{Pr}>\mathrm{F}$ \\
\hline Maintrt & 2 & 17219.22428 & 8609.61214 & & & 6.24 & 0.0199 \\
\hline
\end{tabular}

